And

UNIVERSIDAD AUTÓNOMA METROPOLITANA
UNIVERSIDAD AUTÓNOMA METROPOLITANA - IZTAPALAPA DIVISIÓN DE CIENCIAS BÁSICAS E INGENIERÍA

\title{
DISEÑO DE UN GENERADOR DE \\ PATRONES RESPIRATORIOS VISUALES \\ PARA LA EVALUACIÓN DE LA \\ ARRITMIA SINUSAL RESPIRATORIA
}

\author{
Tesis que presenta \\ Armando Chávez Calderón \\ Para obtener el grado de \\ Maestro en Ciencias en Ingeniería Biomédica
}

\author{
Asesor: Dr. Salvador carrasco \\ Jurado calificador:
}

Presidente: Dra. María del Rocío Ortiz Pedroza

Secretario: Dr. Salvador Carrasco Sosa

Vocal: $\quad$ M. en C. Raúl Martínez Memije

México, D.F., Julio de 2015. 


\section{DEDICATORIA Y AGRADECIMIENTOS}

Mis agradecimientos son para muchas personas que me han acompañado en este trajinar de mi vida, pero en especial agradezco a mi madre la Sra. Ana Ma. Calderón quién me ha apoyado incondicionalmente desde que era un bebe y quién me ha educado de la mejor manera para que sea hoy una persona con altos valores morales y éticos, a ser sensible y consiente de los problemas de los demás y al respeto de toda clase de vida; a mi sobrina Anita por sentirse orgullosa de mi; a mi muy estimada amiga Adriana Pliego con quién pasé gratos momentos con grandiosas risas así como grandes desahogos; a mi excelente amigo Israel Benítez quién siempre me apoyó en esta nueva meta y en otras muchas circunstancias de mi vida y quien me proporcionó su ayuda incondicional en situaciones difíciles al ingresar al posgrado; a mi amiga Karla Rojas por saber escucharme en momentos de tensión con gente insana, por su valiosa amistad y apoyo; a mi colega Ma. Elena Guzmán por su ayuda en momentos difíciles del seminario y a mi extraordinaria Maestra y amiga Dra. Claudia Barbosa quién me ha apoyado en todos los sentidos y en todo momento con su escucha, su apoyo moral y sus sabios consejos. Muchas gracias.

A mi novia Miriam Juárez Anzaldo por recordarme en todo momento que no me distrajera de lo que estaba escribiendo y por su esmerado amor hacia mí.

A mis maestros que con paciencia escucharon mis opiniones y con quienes pasé excelentes momentos de clase, al M. en C. Miguel Cadena, Dr. Juan Carlos Echeverría, Dra. Norma Pilar Castellanos y al Dr. Salvador Carrasco, a todos ellos gracias por sus valiosas enseñanzas.

A mis sinodales: Dra. Ma. del Rocío Ortiz Pedroza y MC Raúl Martínez Memije por sus valiosas enseñanzas.

Al excoordinador de posgrado Dr. Ramón Jiménez por su disposición en las peticiones realizadas.

A mis extraordinarios maestros de idiomas, Beatriz Ramírez, Esperanza Terán, Francisco Sánchez y Daniel Rojas.

A mi amigo y proveedor M. en C. Félix Guerrero por su apoyo y consejos técnicos. A los creadores de Arduino y a Andrew Stone desarrollador de Lightuino.

A Alejandra Guillen y a todas las personas que me prestaron algo de su valioso tiempo para poder realizar los registros fisiológicos del proyecto.

Al CONACyT por su apoyo financiero.

A mi cuerpo por soportar grandes desvelos y ayunos, a mi espíritu por salir avante ante las envidias, arrogancias y egoísmos de los demás.

A mis enemigos pues sus envidias me engrandecieron.

Armando 


\section{CONTENIDO}

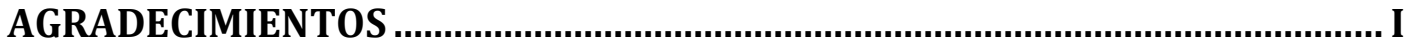

RESUMEN

CAPÍTULO I

INTRODUCCIÓN

I.1 Planteamiento del problema ...................................................................................... 3

I.2 Relevancia de la investigación ................................................................................... 4

I.2.1 Tecnología mininvasiva y sistemas embebidos ………………………………………….... 4

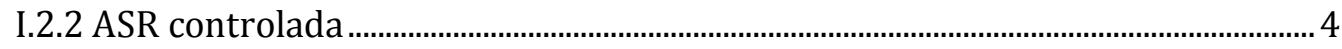

I.2.3 Utilidad clínica bajo condiciones de control respiratorio........................................... 6

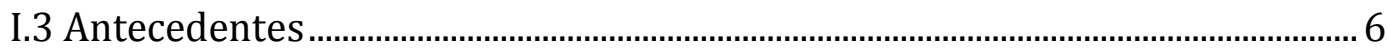

I.3.1 Importancia del uso de instrumentación en el control de la respiración .......... 6

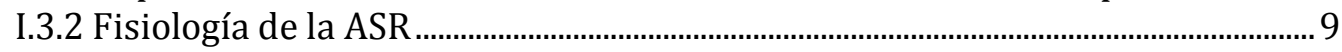

I.3.3 Efectos del control experimental de la respiración................................................10

I.3.4 Variabilidad de la frecuencia cardiaca: VFC ...........................................................11

I.3.5 Utilidad clínica de la VFC..........................................................................................11

I.3.6 Comportamiento espectral de la VFC .................................................................11

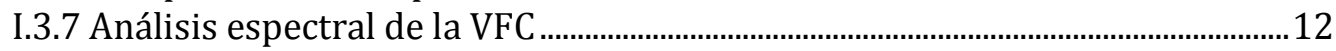

I.4 Estado de la técnica ..................................................................................................... 13

I.4.1 Métodos e instrumentos para el control de la respiración ...................................13

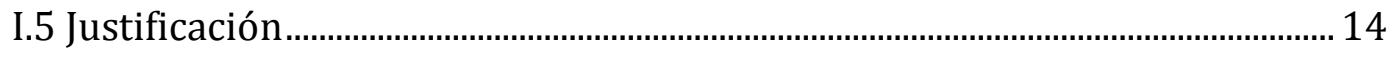

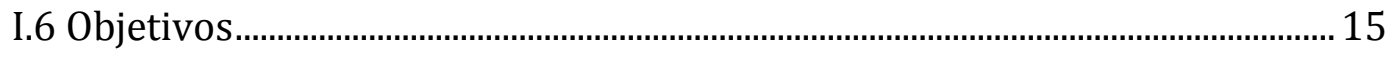

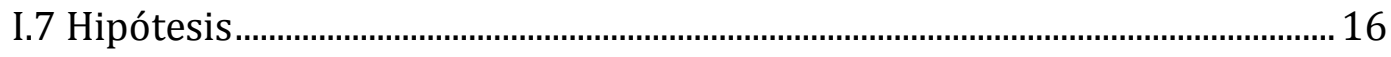

$\begin{array}{ll}\text { CAPÍTULO II } & \mathbf{1 7}\end{array}$

$\begin{array}{ll}\text { METODOLOGÍA } & 17\end{array}$

II.1 Especificaciones de diseño del GPRV ................................................................ 18

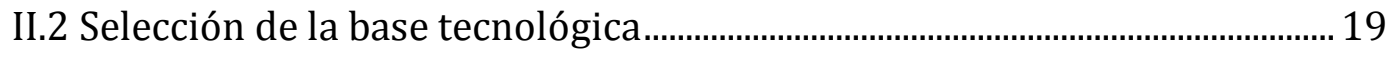




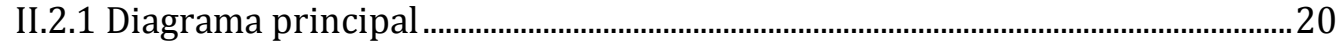

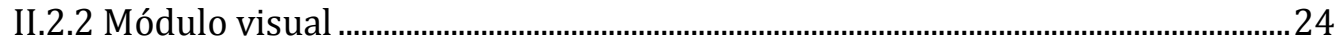

II.2.3 Diseño de la etapa de acondicionamiento del neumotacómetro al GPRV ....25

II.2.4 Procesamiento de la señal en el sistema embebido .............................................27

II.3 Ajuste de los parámetros fisiológicos al GPRV ……………………………….... 27

II.3.1 Ajuste de las funciones programadas del parámetro fisiológico frecuencia

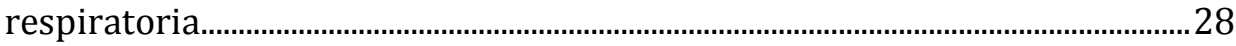

II.3.1.1 Calibración del GPRV en frecuencia ....................................................................33

II.3.2 Ajuste de las funciones programadas del parámetro fisiológico amplitud

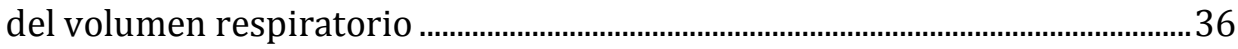

II.3.2.1 Calibración del parámetro amplitud del volumen respiratorio .........................38

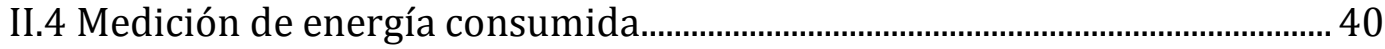

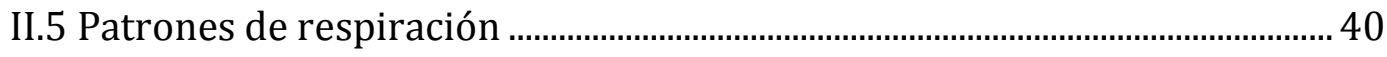

II.5.1 Patrón de respiración Amplitud Constante Frecuencia Constante ACFC .....40

II.5.2 Patrón de respiración Amplitud Variable Frecuencia Constante AVFC..........40

II.5.3 Patrón de respiración Amplitud Constante Frecuencia Variable ACFV ..........41

II.6 Validación del GPRV ............................................................................................... 42

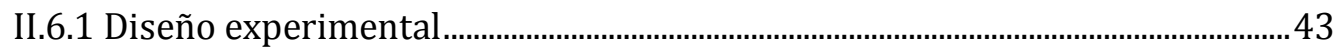

II.6.1.1 Sujetos de experimentación................................................................................. 43

II.6.1.2 Protocolo de esfuerzo respiratorio................................................................... 44

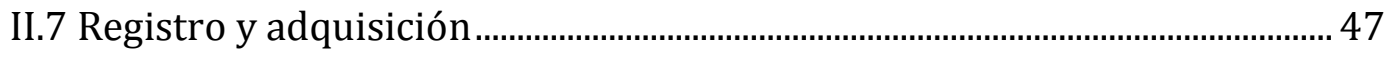

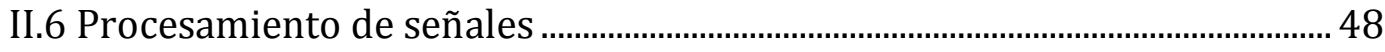

$\begin{array}{lr}\text { CAPÍTULO III } & 49\end{array}$

$\begin{array}{lr}\text { RESULTADOS } & \mathbf{5 0}\end{array}$

III.1 Costos del sistema embebido .......................................................................... 50

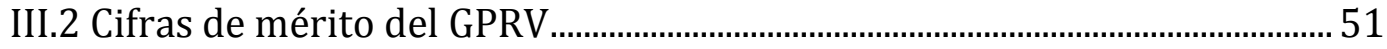

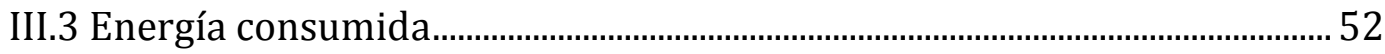

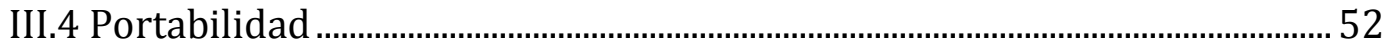


III.5 Comparación de técnicas de caracterización por GPRV y computadora.....53

DISCUSIÓN 58

III.6 Principales hallazgos .................................................................................................... 58

III.7 Aportes al conocimiento fisiológico …………………….................................. 59

$\begin{array}{ll}\text { CAPÍTULO IV } & \mathbf{6 0}\end{array}$

$\begin{array}{ll}\text { CONCLUSIONES } & 61\end{array}$

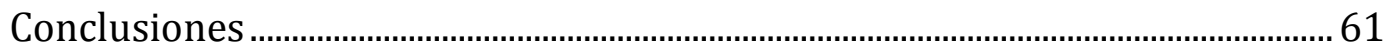

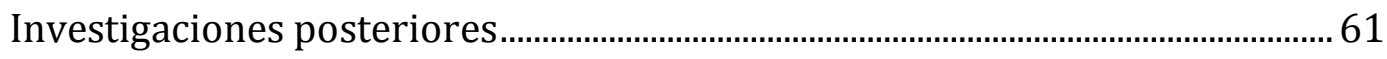

Otras posibles áreas de beneficio ............................................................................. 62

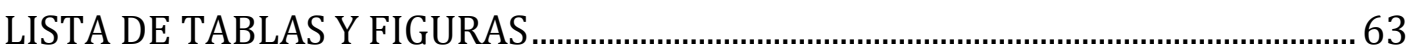

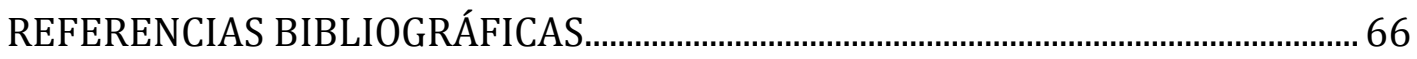

ANEXO 1

Ajuste de parámetros fisiológicos

ANEXO 2

Ambigüedad referente a mantener la frecuencia constante con respecto a la amplitud variable 


\section{RESUMEN}

El control de la respiración se ha usado exhaustivamente en muchas investigaciones en el mundo como modulador de las ramas nerviosas simpática y parasimpática con el fin de caracterizar la Arritmia Sinusal Respiratoria. El Laboratorio de Fisiología Médica de la Universidad Autónoma Metropolitana Iztapalapa ha utilizado algunas tecnologías para el control respiratorio, como lo son marcadores de tiempo audibles, marcadores de tiempo visuales con columnas de LED y por visualización de patrones en una pantalla; en el caso de los marcadores por tonos audibles quedaron descartados ya que presentaban errores absolutos grandes en frecuencia de $\pm 0.19 \mathrm{~Hz}$, los dispositivos por columna de LED fueron validados como aceptables, sin embargo carecen de la posibilidad de generar patrones con barridos en frecuencia y amplitud, carecen de portabilidad y no tienen entrada analógica para el seguimiento de los sujetos en tiempo real; por otro lado el método por computadora con seguimiento visual de los patrones en una pantalla LCD se encuentra sujeto a un hardware excesivo, consumo de energía de aproximadamente de $120 \mathrm{~W}$ por computadora, limitado en movimiento y costoso por el uso de licencias diversas de software; de esta manera surgió la necesidad de diseñar un nuevo instrumento visual con columnas de LED con ventajas como generación por software de diversos patrones respiratorios con frecuencias y amplitudes fijas y variables (barridos en frecuencia y amplitud), hardware portable, consumo bajo de energía, modos de ahorro de energía (estado en espera cada que termina un protocolo), controlado remotamente hasta una distancia de $10 \mathrm{~m}$ por medio de un control remoto infrarrojo y no tiene costos de licencias. Los objetivos de esta tesis se centraron en tener un instrumento capaz de generar patrones respiratorios con amplitudes y frecuencias fijas así como amplitudes y frecuencias variables pero con su correspondiente calibración en cada patrón con lo que se obtuvieron cifras de mérito aceptables de 0.992 para precisión, 0.985 para exactitud y 0.998 para certeza respecto de la variable frecuencia, con respecto a la amplitud se obtuvieron cifras de mérito aceptables de 0.991 para precisión, 0.986 para exactitud y 0.996 para certeza; y su correspondiente validación que sustentó su utilidad fisiológica por medio de un diseño experimental donde se aplicó un protocolo de esfuerzo respiratorio a 11 sujetos de genero indistinto con tres patrones de respiración con duración de 1 minuto: Amplitud Constante Frecuencia Constante (1 litro, $0.2 \mathrm{~Hz}$ ), Amplitud Constante Frecuencia Variable en incremento (1 litro, barrido en frecuencia lineal de 0.15 a $0.6 \mathrm{~Hz}$ ), Amplitud Variable en incremento Frecuencia Constante (barrido en amplitud de 0.2 a 2 litros, $0.2 \mathrm{~Hz}$ ). Para cada patrón respiratorio se midieron los errores absolutos de amplitud y frecuencia, de respiración a respiración entre el Generador de Patrones Respiratorios Visuales (GPRV) y el patrón 
generado en MatLab y entre un método comprobado, por computadora con visualización en pantalla TFT y el patrón generado en MatLab.

Las mediciones demostraron que los valores medios del error absoluto de las variables amplitud del volumen respiratorio y frecuencia respiratoria entre el método de referencia y el Generador de Patrones Respiratorios Visuales fueron no significativos: en la comparación entre el Generador de Patrones Respiratorios Visuales y la computadora respecto del patrón generado en MatLab se encontró para el patrón Amplitud Constante Frecuencia Constante (error GPRV $0.02 \mathrm{~Hz} \pm 0.03$, error computadora $0.03 \mathrm{~Hz} \pm 0.03$; error GPRV $42 \mathrm{ml} \pm 46 \mathrm{ml}$, error computadora $34 \mathrm{ml} \pm 30 \mathrm{ml}$ ); para el patrón Amplitud Constante Frecuencia Variable en incremento (error GPRV $0.08 \mathrm{~Hz}$, error computadora $0.08 \mathrm{~Hz}$; error GPRV $77 \mathrm{ml} \pm 62 \mathrm{ml}$, error computadora $57 \mathrm{ml} \pm 45 \mathrm{ml}$ ); para el patrón Amplitud Variable en incremento Frecuencia Constante (error GPRV $0.02 \mathrm{~Hz}$, error computadora $0.03 \mathrm{~Hz}$; error GPRV $84 \mathrm{ml} \pm 75 \mathrm{ml}$, error computadora $57 \mathrm{ml} \pm 62 \mathrm{ml}$ ).

En el análisis los valores medios de la ASR fueron muy similares entre las tres maniobras: para el patrón Amplitud Constante Frecuencia Constante (GPRV 145 \pm 63 , computadora 146 \pm 42 ); para el patrón Amplitud Constante Frecuencia Variable en incremento (GPRV $87 \pm 28$, computadora $86 \pm 33$ ) y para el patrón Amplitud Variable en incremento Frecuencia Constante (GPRV 132 \pm 56 , computadora $141 \pm 45$ ).

Los resultados de esta tesis indican que el Generador de Patrones Respiratorios Visuales, logró reproducir adecuadamente patrones respiratorios en amplitud y frecuencia tanto constantes como variables, además mostró ser adecuado al incrementar o decrementar la función de la rama vagal cardiaca.

\section{Palabras clave:}

Generador de Patrones Respiratorios Visuales (GPRV).

Arritmia Sinusal Respiratoria (ASR).

Sistema embebido.

Convertidor analógico digital CAD.

Control de la respiración.

Variabilidad de la frecuencia cardiaca (VFC).

Amplitud del volumen de la respiración (AVOR).

Sistema Nervioso Autónomo (SNA).

Núcleos Generadores de Patrones respiratorios (NGPR).

Núcleos Nerviosos Vagales (NNV).

Baro receptores (BARORx).

Volumen corriente (VC). 
Número de respiraciones por minuto (Nrpm).

Amplitud Constante Frecuencia Constante (ACFC).

Amplitud Variable en incremento Frecuencia Constante (AViFC).

Amplitud Constante Frecuencia Variable en incremento (ACFVi). 


\section{CAPÍTULO I INTRODUCCIÓN}

"Creo firmemente que la gente debe beneficiarse con mi formación".

- Dr. Shima Gyoh | Nigeria 


\section{CAPÍTULO I INTRODUCCIÓN}

\section{I.1 Planteamiento del problema}

El control de la respiración se ha usado exhaustivamente en muchas investigaciones en el mundo como modulador de las ramas nerviosas simpáticas y parasimpáticas con el fin de caracterizar la Arritmia Sinusal Respiratoria (ASR). Para ello se han utilizado diferentes métodos para realizar el control de la respiración como lo son con tonos audibles marcados por metrónomos mecánicos, electrónicos y por software; visualización de patrones como dispositivos con columnas de LED y un método utilizado en el Laboratorio de Fisiología Médica de la Universidad Autónoma Metropolitana Iztapalapa por visualización en una pantalla de TFT de diferentes patrones generados en MatLab. Sin embargo se encontró que los métodos audibles son bastante difíciles de seguir por los sujetos, condición que se midió cualitativamente con encuestas a los sujetos bajo prueba y cuantitativamente por medio de la medición del error con respecto a un patrón generado en MatLab. En un estudio piloto previo realizado en el laboratorio de Fisiología Médica con una muestra de 8 sujetos se compararon tres métodos, el audible, el dispositivo de columna de LED, y el de computadora, este consistió de un patrón ACFC con amplitud constante de 2 litros y frecuencia de $0.2 \mathrm{~Hz}$ y presentaron un error absoluto promedio de $0.19 \mathrm{~Hz}$ respecto de la frecuencia y los dispositivos visuales por columna de LED un error absoluto promedio de $0.09 \mathrm{~Hz}$ con referencia a la frecuencia, además están muy limitados pues sólo pueden marcar tiempos a frecuencias fijas y amplitudes del volumen constantes, y sólo uno de ellos el último diseño se desarrolló con una entrada analógica para las señales de respiración de los sujetos ${ }^{1}$. El método de computadora presentó un error de $0.04 \mathrm{~Hz}$.

Las dos generaciones de metrónomos visuales con columnas de LED basados en microcontrolador fueron diseñados con el fin de darle portabilidad (que pueda llevarse el dispositivo a otro lado del laboratorio) y flexibilidad (que la columna de LED pueda colocarse en diversas posiciones) con el objetivo de hacer mediciones en diferentes posiciones del sujeto bajo prueba ya que la respuesta de los núcleos

\footnotetext{
${ }^{1}$ Valores de los errores absolutos tomados de la comparación de métodos de control respiratorio realizados en 2012 en estudios piloto en el laboratorio de Fisiología Médica de la UAM-I. 
generadores de patrones respiratorios es distinta dependiendo de la posición del sujeto, sin embargo no se logró ese objetivo ya que dependen de una fuente de alimentación fija y además sólo son marcadores de tiempo pues no se reporta un estudio estadístico que caracterice al dispositivo en cuanto a precisión y exactitud, sólo se reportan los efectos al proceso fisiológico. Dado que los efectos al proceso fisiológico son reportados como útiles desde el punto de vista del impacto en la ASR [1] los dispositivos son validados.

El tercer método utilizado para la investigación dentro del laboratorio de Fisiología Médica de la UAM-I es tomado como método de referencia, pues éste genera patrones cuasi-continuos de respiración que son representados en una pantalla de TFT de 32 pulgadas, los cuales deben ser seguidos por los sujetos bajo prueba, en estudios piloto previos presentaron un error absoluto en frecuencia de \pm 0.1 , sin embargo este método se encuentra sujeto a un hardware excesivo pues se requieren dos computadoras de escritorio personales y dos pantallas de TFT de 32 pulgadas, tiene un consumo de energía considerable de aproximadamente 120W, está limitado en movimiento (es decir que los sujetos bajo prueba sólo pueden estar en posiciones: sentado y parado, no en decúbito supino o dorsal u otras posiciones), genera patrones de barrido en amplitud del volumen respiratorio relativamente difícil de seguir a criterio de los sujetos debido a que no se pueden anticipar al valor máximo siguiente en cada ciclo de respiración y además son de costo considerable por el uso de software de licencia como lo es Windows ${ }^{\mathrm{TM}}$ de \$2099MX y MatLab ${ }^{\circledR}$ de \$9742MX lo que implica un mayor costo económico.

Estas limitaciones de los sistemas con columna de LED y con patrones generados con MatLab impulsan la búsqueda de nuevos instrumentos que sean capaces de generar patrones respiratorios con valores en amplitud y frecuencia constantes, barridos en frecuencia y amplitud, con hardware portable, menor consumo de energía, flexible en cuanto a movimiento y con entrada analógica para el monitoreo y seguimiento de la respiración de los sujetos bajo experimentación, tanto para el área de investigación en laboratorio como en perspectiva para su uso en la clínica con pacientes ambulatorios. 


\section{I.2 Relevancia de la investigación}

\section{I.2.1 Tecnología mininvasiva y sistemas embebidos}

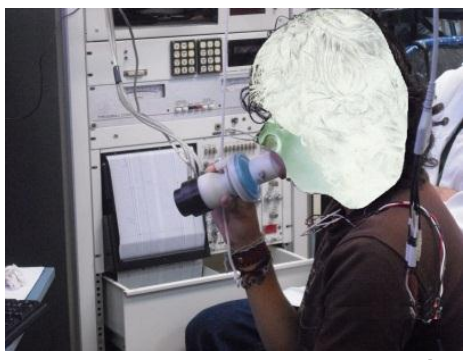

Figura 1.1. Caracterización de la ASR mininvasiva.
El uso de tecnología mínimamente invasiva en los procesos de investigación referentes a la Arritmia Sinusal Respiratoria (ASR) en el área de Fisiología Humana es fundamental. Ésta permite tener un menor estrés sobre los sujetos de estudio y un mejor control sobre la variable de interés: la respiración. Figura 1.1.

Hace una década el uso de sistemas embebidos, para coadyuvar en dicha tarea comenzó a hacerse notar. Por ende en el Laboratorio de Fisiología Médica de la Universidad Autónoma Metropolitana Unidad Iztapalapa (UAM-I) en el área de investigación referente al impacto que tiene la ASR sobre la actividad vagal se comenzó a usar este tipo de tecnología. Figura 1.2

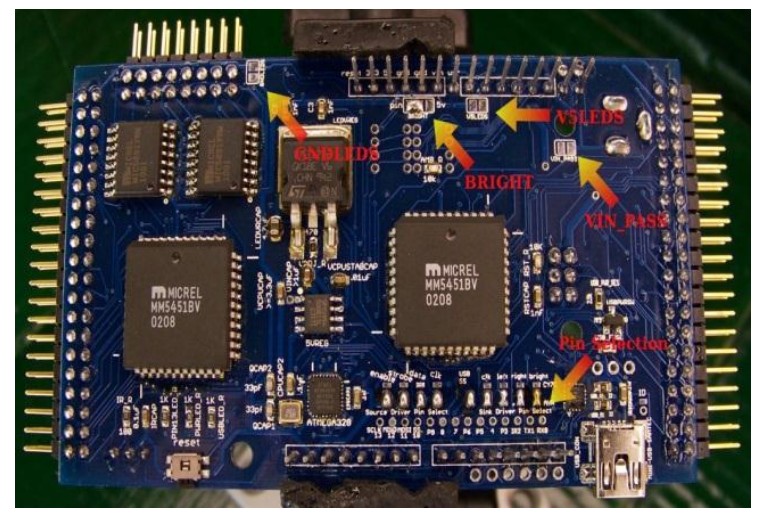

Figura 1.2. Sistema embebido Lightuino ${ }^{\mathrm{TM}}$.

\section{I.2.2 ASR controlada}

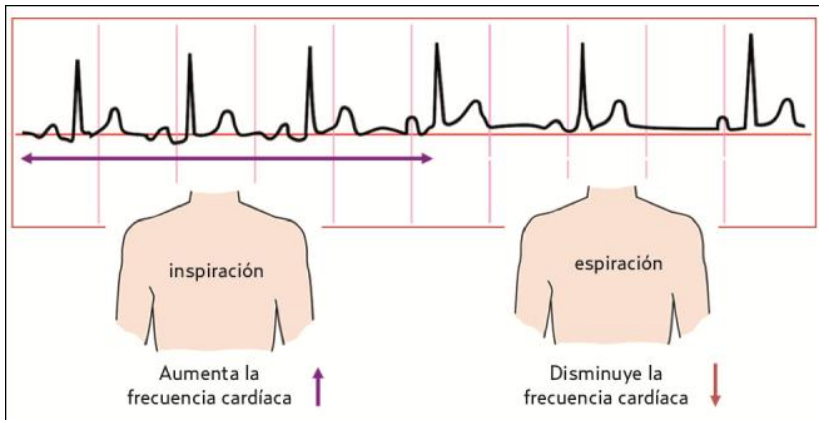

Figura 1.3. Importancia del control de la respiración.
La importancia del control de la respiración en esta área de investigación es de mayúsculo interés. Se sabe que la respiración en la inspiración provoca que la frecuencia cardiaca aumente y que durante la espiración la frecuencia disminuya. Este fenómeno de sincronización de la respiración con la frecuencia cardiaca se le conoce como arritmia sinusal respiratoria (ASR). Figura 1.3. Este control de la respiración probado experimental $\mathrm{y}$ exhaustivamente consiste en tres protocolos 
característicos: el primero es hacer que el sujeto controle su respiración manteniéndola lo más constante posible tanto en frecuencia como en volumen, el segundo consigue hacer que el sujeto haga variar su respiración al incrementar linealmente su frecuencia respiratoria manteniendo el volumen de su respiración lo más constante posible, el tercero implica que el sujeto mantenga lo más constante posible su respiración en frecuencia e incremente su volumen de forma lineal. Con estos protocolos se logra estimular con mayor impacto la rama nerviosa parasimpática o vagal, con lo que es posible caracterizar la ASR.

Esta caracterización de la ASR es importante también en la clínica ya que es una respuesta fisiológica que supone la no existencia de patologías [2].

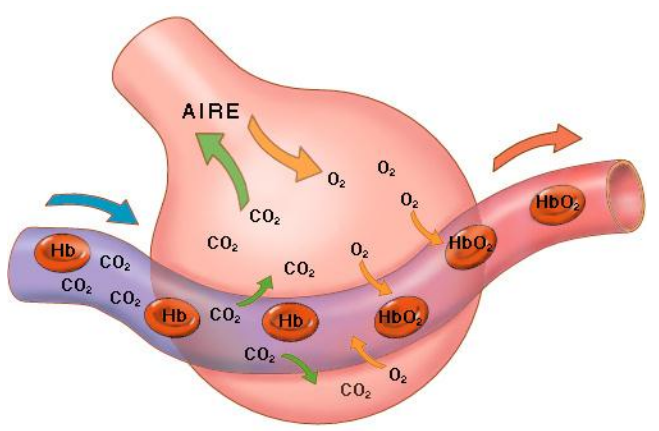

Figura 1.4. Intercambio de gases pulmonares a nivel alveolar.

Estudios recientes han demostrado que la eficiencia del intercambio de gases pulmonares mejora por medio de una ASR controlada, figura 1.4. Esto indica que la ASR controlada desempeña un papel fisiológico activo. De esta manera la ASR controlada tiene una influencia positiva en el intercambio ventilación-perfusión a nivel pulmonar [3].

Los protocolos de respiración mayormente usados son:

1- ACFC (1 litro y $0.2 \mathrm{~Hz}$ );

2- ACFVi (amplitud constante de 1 litro y frecuencia variable en incremento lineal de 0.2 a $0.6 \mathrm{~Hz}$ );

3- AViFC (amplitud creciente lineal de 0.2 a 2 litros y frecuencia constante de $0.2 \mathrm{~Hz}$ )

A diferentes tiempos de duración, predominando los de corta duración de 1 minuto.

Estos se usan debido al intenso estímulo que logran hacia la rama parasimpática. 


\section{I.2.3 Utilidad clínica bajo condiciones de control respiratorio}

Su utilidad clínica se aplica en algunas condiciones referentes a la valoración de riesgo en cardiopatía isquémica, vigilancia de la falla cardiaca, diagnóstico temprano de neuropatía diabética [4].

Actualmente se encuentra bajo prueba en la evaluación del rendimiento deportivo, alteraciones del sueño, desórdenes alimenticios, enfermedades psiquiátricas, alteraciones fetales, sincopé y otros procesos fisiológicos en dónde se les ha encontrado relación importante con el Sistema Autónomo [4].

\section{I.3 Antecedentes}

\section{I.3.1 Importancia del uso de instrumentación en el control de la respiración}

El aporte científico más antiguo que se encontró respecto de la importancia del control respiratorio para relacionar la Arritmia Sinusal Respiratoria (ASR) con la frecuencia cardiaca fue en 1964, cuando Angelone y Coulter en su trabajo "Respiratory sinus arrhythmia: a frequency dependent phenomenon" [5], hicieron variar la respiración de un sujeto sano de 36 años sobre un amplio rango de frecuencia, observando las fluctuaciones del ritmo cardiaco. El instrumento diseñado y construido para el control de la respiración fue un generador de pulsos audible de 30 a 180 pulsos por minuto.

Lo que les permitió encontrar una relación entre la amplitud del ritmo cardiaco y la frecuencia de la respiración y así determinar que la ASR se presentaba en sujetos de cualquier edad y no sólo en niños y jóvenes adultos como se reportaba. Además de determinar a la ASR como un fenómeno dependiente de la frecuencia. 
Después en 1981 Hirsch y Bishop en su artículo "Respiratory sinus arrhythmia in humans: how breathing pattern modulates heart rate" [6], reportaron un experimento con 17 sujetos a los que se les hizo variar su respiración en frecuencia a diferentes amplitudes del volumen respiratorio.

La técnica reportada para que los sujetos mantuvieran una frecuencia respiratoria más o menos estable fue utilizar un metrónomo mecánico

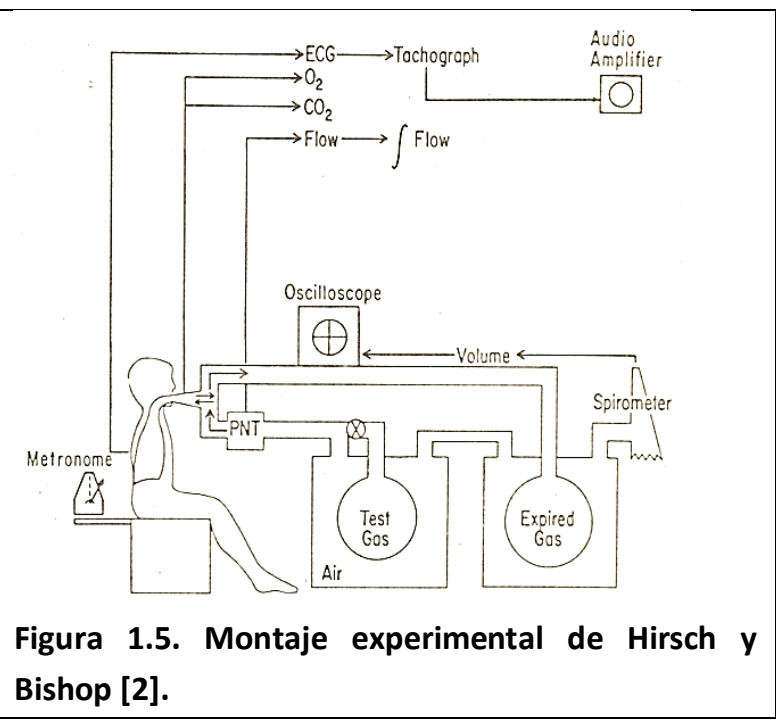
y para seguir la amplitud del volumen se usó un espirómetro. Figura 1.5.

Se encontraron comportamientos como filtro pasa-bajas entre distintos valores de volumen corriente y el incremento de la frecuencia respiratoria en sujetos jóvenes.

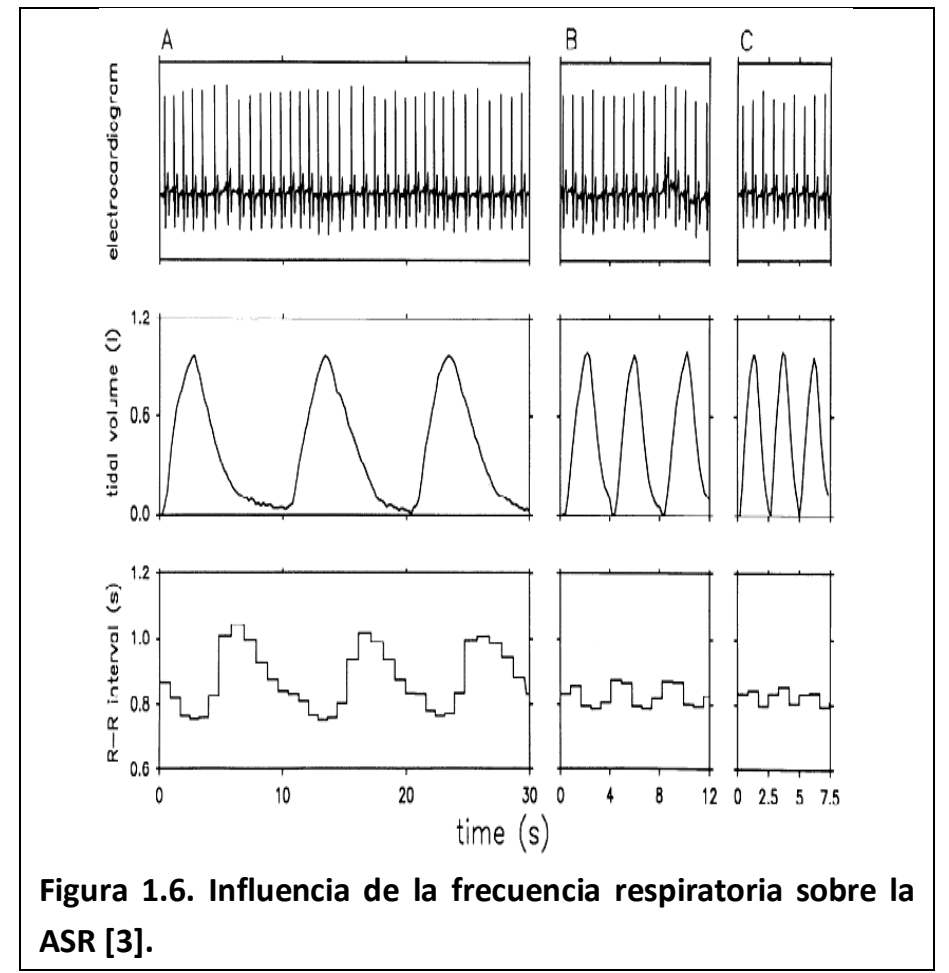

Posteriormente en 1993 Brown y cols. en su artículo "Important influence of respiration on human R-R interval power spectra is largely ignored"[7], reportaron un estudio hecho a nueve sujetos sanos en decúbito supino con distintos ritmos respiratorios de 6 a 24 respiraciones por minuto a dos amplitudes constantes del volumen corriente, $1,000 \mathrm{y}$ 1,500 ml. El método reportado para el control de la respiración fue por medio de una señal auditiva de 3 a

24 respiraciones por minuto. En el cual encontraron una fuerte influencia de la frecuencia respiratoria y el volumen corriente sobre la ASR. Fig. 1.6. 
Luego en 2001 Carrasco y cols. en "Dispositivo electrónico para el control de la frecuencia respiratoria: Impacto en la reproducibilidad de la variabilidad de la frecuencia cardiaca" [1], publicaron un estudio hecho a 11 sujetos sanos que mantuvieron una frecuencia respiratoria de $0.2 \mathrm{~Hz}$. Se reporta el uso de un dispositivo electrónico para el control de la respiración, basado en microcontrolador con barra de 60 LED, figura 1.7, para

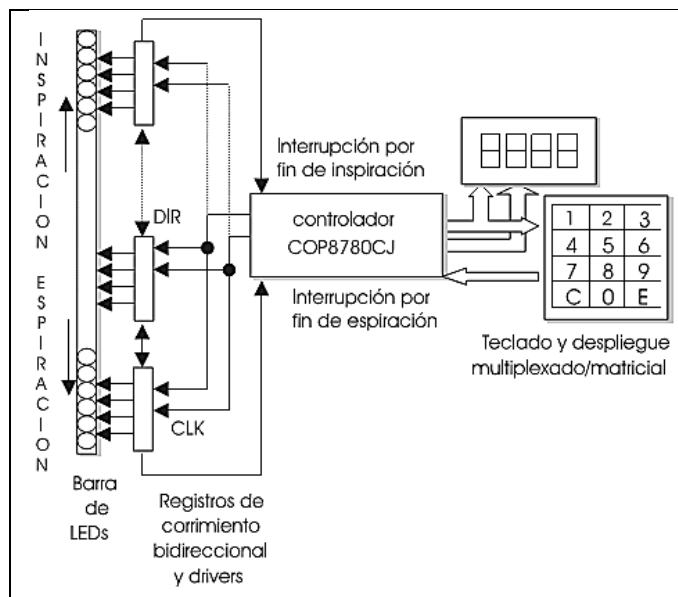

Figura 1.7. Dispositivo electrónico con barra de LED. mostrar patrones de amplitud del volumen respiratorio constante y diferentes frecuencias programables.

Se encontraron mejoras sustanciales del dispositivo con barra de LED en cuanto al mejor control de la frecuencia respiratoria de los sujetos bajo estudio en comparación con los métodos tradicionales audibles y mejoras en la reproducibilidad de los índices temporales y espectrales de la ASR.

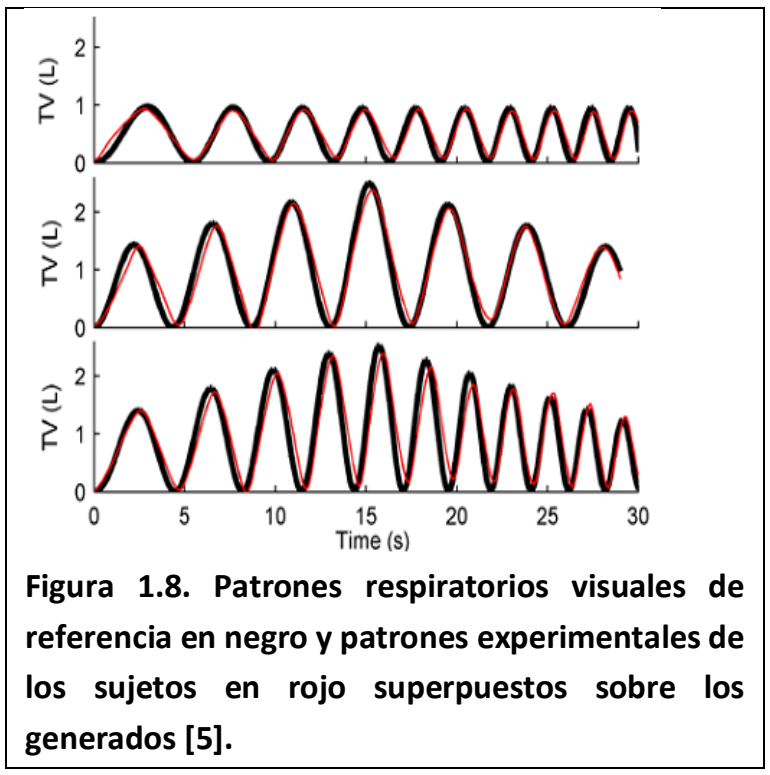

Finalmente en 2012 Guillen y Carrasco en "Interactive Effects of Simultaneously Varying Respiratory Frequency and Tidal Volume on Respiratory Sinus Arrhythmia" [8], reportaron en Computing in Cardiology la ejecución de un procedimiento basado en maniobras respiratorias guiadas por patrones respiratorios visuales mostrados en una pantalla TFT (Thin Film Transistor). No se reportó el uso del sistema pero se sabe que éste método utiliza dos computadoras

personales y dos pantallas TFT de 32 pulgadas, así como software costoso como lo es licencias de Windows ${ }^{\mathrm{TM}} \mathbf{y}_{\mathbf{M a t L a b}^{\mathrm{TM}}}$. La ejecución de estas maniobras fue realizada por 25 sujetos por medio de tres protocolos lineales de respiración durante 30s; amplitud constante del volumen de $1 \mathrm{~L}$ (TV) y frecuencia variable en incremento de 0.15 a $0.5 \mathrm{~Hz}$ (ACFVi); amplitud del volumen variable en incremento y 
decremento de 1 a $2.5 \mathrm{~L}$ y 2.5 a $1 \mathrm{~L}$ con frecuencia constante de $0.2 \mathrm{~Hz}$ (AVidFC) y amplitud variable en incremento y decremento de 1 a $2.5 \mathrm{~L}$ y 2.5 a $1 \mathrm{~L}$ con frecuencia variable en incremento de 0.2 a $0.5 \mathrm{~Hz}$ (AVidFVi). Se encontraron relaciones continuas, lineales e inversas con el incremento de la frecuencia respecto de la ASR y relaciones complejas con histéresis entre la amplitud del volumen en incremento y decremento y la ASR. Estas relaciones muestran fuertes efectos de la respiración controlada sobre la arritmia sinusal respiratoria (ASR), figura 1.8.

\section{I.3.2 Fisiología de la ASR}

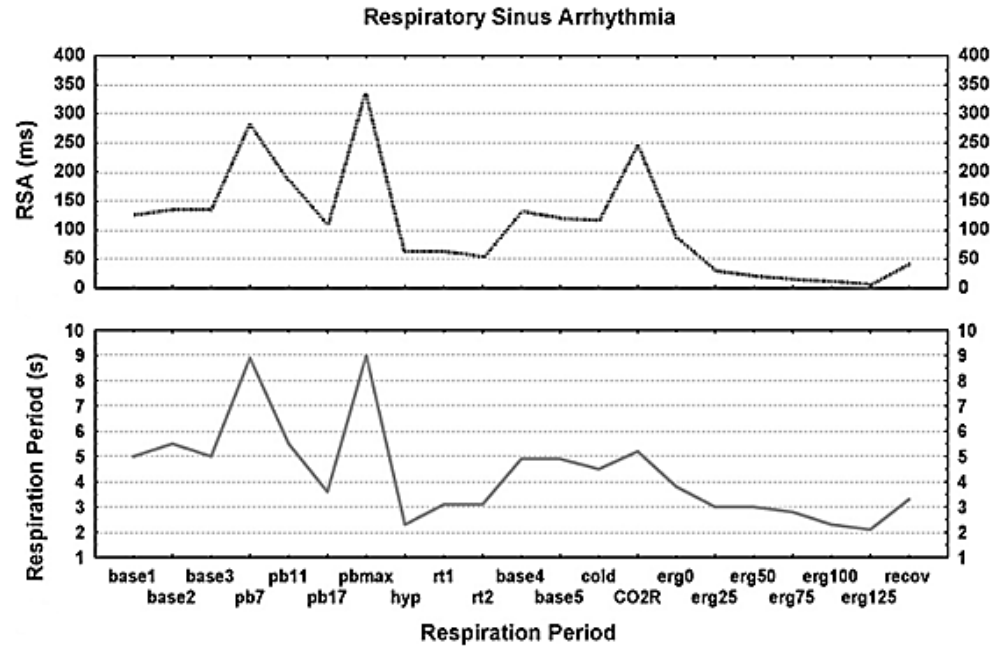

Figura 1.9. Patrones similares del periodo de la respiración y la ASR de diferentes tareas de esfuerzo respiratorio [6].
La ASR es un fenómeno fisiológico en donde existe una gran interacción entre el sistema respiratorio y el cardiovascular [9]. Esta interacción se debe a la estimulación causada por la respiración principalmente sobre la actividad vagal. Al influir principalmente sobre la actividad vagal, la respiración se

sincroniza con la frecuencia cardiaca [3]. Esta sincronía provoca cambios en los periodos cardiacos dando lugar a una variabilidad de la frecuencia cardiaca (VFC). Figura 1.9.

La manera en que la respiración influye sobre la frecuencia cardiaca ocurre durante la inspiración y la espiración dando lugar a un aumento y una disminución de la frecuencia cardiaca respectivamente. Estos cambios que suceden de manera espontánea también se pueden ver afectados por los efectos de una respiración controlada.

La respiración controlada puede influir directamente sobre el nervio vago dando lugar a descargas del nervio vago que pueden producir un aumento o disminución de la amplitud de la ASR. Figura 1.10. 


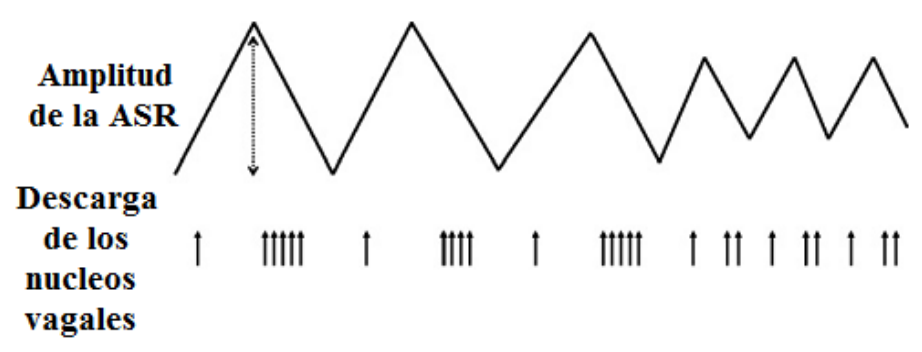

Figura 1.10. Modelo simulado de la relación entre la ASR y las descargas del nervio vago [6].

Además el seguimiento visual de un patrón determinado de respiración por los sujetos es muy simple de hacer. Por lo tanto la ASR presenta bondades que le hacen adecuada para ser usada como un índice del control vagal cardiaco [2].

La ASR puede cuantificarse de diferentes maneras entre ellas: análisis espectral, análisis en el dominio del tiempo por picos-valles o aplicaciones como filtro pasabanda [2].

\section{I.3.3 Efectos del control experimental de la respiración}

El hecho de que los sujetos sigan ciertos patrones respiratorios nos da una mejor comprensión del funcionamiento del balance tan sutil que existe entre las ramas nerviosas simpática y parasimpática o vagal. Las cuales son ejecutoras del control que lleva a cabo el Sistema Nervioso Autónomo sobre el Sistema Cardiovascular.

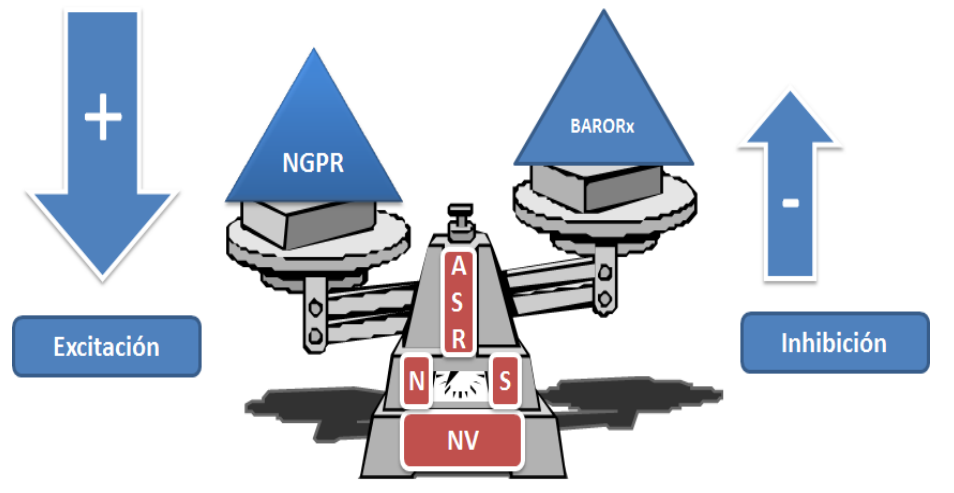

Figura 1.11. Influencia de los Núcleos generadores de patrones respiratorios (NGPR) sobre la ASR. BaroRx: baroreceptores, NV: núcleos vagales, NS: nodo sinusal.
El control experimental de la respiración ayuda a tener un mejor dominio sobre las variables frecuencia y amplitud del volumen que afectan principalmente a los núcleos nerviosos vagales, que a su vez estimulan el nodo sinusal y éste impacta directamente sobre el comportamiento de la ASR. Figura 1.11. 


\section{I.3.4 Variabilidad de la Frecuencia Cardiaca: VFC}

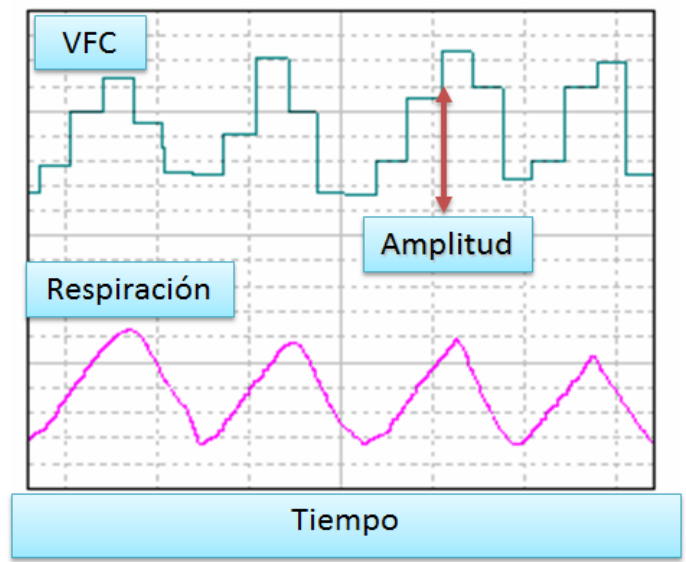

Figura 1.12. Construcción de la serie de tiempo VFC.
La técnica más común para poder medir estos cambios en la frecuencia cardiaca es por medio de la creación de un tacograma. Dicho tacograma es un gráfico que muestra el comportamiento de la frecuencia cardiaca en el tiempo. Este gráfico se construye a partir de la serie temporal de intervalos R-R capturada previamente por medio de un electrocardiógrafo [10].

A grandes rasgos la construcción de esta serie de tiempo llamada VFC se realiza colocando las diferencias de tiempo entre los intervalos de la serie R-R (variable independiente) como cambios en amplitud (variable dependiente), es decir como coordenadas verticales en el nuevo gráfico tacograma. Figura 1.12.

\section{I.3.5 Utilidad clínica de la VFC}

La utilidad clínica de la VFC se manifiesta notablemente en diagnosis de algunos padecimientos cardiovasculares [11], tales como la valoración de riesgo en cardiopatía isquémica, seguimiento de la falla cardiaca y diagnóstico temprano de la neuropatía diabética [11]. Fig. 1.13.

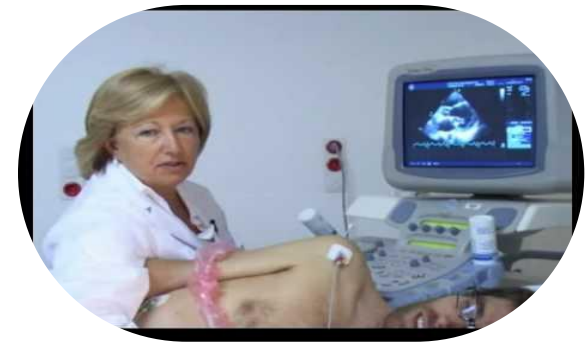

Figura 1.13. Diagnosis de padecimientos cardiovasculares.

\section{I.3.6 Comportamiento espectral de la VFC}

La serie de tiempo VFC es además analizada por medio de su comportamiento espectral, arrojando dos bandas en frecuencia definidas como de Baja Frecuencia (LF: por su acrónimo en inglés "Low Frequency") y Alta Frecuencia (HF: por su acrónimo en inglés "High Frequency"), 0,04 a 0,15 Hz y 0,15 a 0,40 Hz respectivamente [12]. Figura 1.14. 


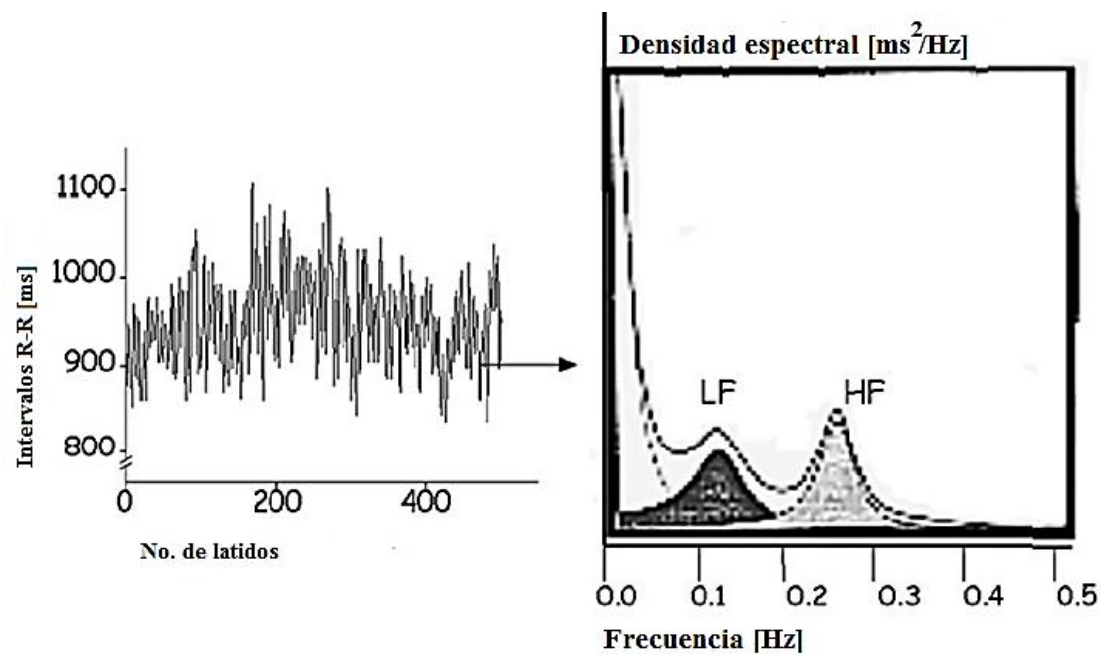

Figura 1.14. Comportamiento espectral de la VFC.

La banda de frecuencia a la que se le ha asociado los efectos de los movimientos respiratorios es la de Alta frecuencia (HF). Esto se debe, desde el punto de vista evolutivo fisiológico, a que el control de la respiración tiene un efecto directo sobre la rama del nervio parasimpático o vagal misma que se ha reportado ampliamente como moduladora de la VFC [11].

\section{I.3.7 Análisis espectral de la VFC}

El objetivo del análisis espectral es cuantificar la densidad espectral de potencia de un proceso estocástico a partir de las muestras de una señal determinada. Este análisis suele clasificarse en métodos paramétricos y no paramétricos. El primero consiste en suponer que el proceso estocástico puede ser caracterizado por una serie de parámetros, un ejemplo típico de este método es el modelo autorregresivo. Mientras que el segundo cuantifica la covarianza asumiendo que el proceso estocástico no presenta ninguna estructura, un ejemplo clásico de este estimador es el periodograma o transformada de Foürier [13].

El análisis de la VFC se ha llevado a cabo por medio de ambas técnicas sin embargo la aplicación de estos métodos es limitada bajo las condiciones de este tipo de series de tiempo. Un problema es su condición no estacionaria que presenta dificultades en el sesgo y la varianza.

Por ello está siendo mayormente preferible para el análisis de estas señales, el uso de Distribuciones Tiempo-Frecuencia (DTF) $[13,14]$. 


\section{I.4 Estado de la Técnica}

\section{I.4.1 Métodos e instrumentos para el control de la respiración}

En la revisión bibliográfica más reciente se encontraron sólo tres publicaciones importantes y un proyecto de licenciatura acerca del estudio de la ASR por medio del control de la respiración.

El primero del año 2001 "Dispositivo electrónico para el control de la frecuencia respiratoria: Impacto en la reproducibilidad de la variabilidad de la frecuencia cardiaca" [1] en donde se reporta el uso de un dispositivo basado en microcontrolador provisto de una columna de 60 LED que muestra ritmos respiratorios a una frecuencia fija, no se reporta la resolución en amplitud del volumen ni el tamaño de la columna de LED. No se trata de un dispositivo con funciones matemáticas programadas en software sino simplemente un conjunto de valores en tablas que son leídos en un ciclo ni tampoco se informa acerca de los retrasos que ocurren tanto a nivel de software como por el uso de circuitos externos con tecnología TTL. Por lo que no puede ser considerado un instrumento como tal.

El segundo en 2003 "Desarrollo de un metrónomo digital basado en el microcontrolador PIC16F873" realizado como proyecto de licenciatura en la Universidad Autónoma Metropolitana Unidad Iztapalapa, es un marcador de tiempos basado en microcontrolador PIC muy similar al primero excepto que cuenta con entrada analógica, la diferencia radica en que para la obtención de las distintas frecuencias se programaron temporizadores del microcontrolador y además la barra de LED de despliegue de ritmos de frecuencias es de 64 LED y la analógica de visualización del sujeto es de 80 LED. No se hace mención de su calibración ni de la resolución en amplitud.

El tercer estudio importante publicado en 2007 "Toward understanding respiratory sinus arrhythmia: Relations to cardiac vagal tone, evolution and biobehavioral functions" [9] no hace mención del instrumento utilizado para el control de la respiración.

La cuarta y última publicación en 2012 "Interactive effects of simultaneously varying respiratory frequency and tidal volume on respiratory sinus arrithmia" [8] 
tampoco reporta el uso de la instrumentación para el control de la respiración. Sin embargo con el permiso de los autores y debido a que el proyecto de tesis del diseño de un GPRV fue realizado en el mismo laboratorio se pudo acceder a la información acerca de cómo se realiza el control de la respiración.

El hardware y los procesos generales asociados para el control de la respiración se realizan de la siguiente manera, ver figura 1.15 , se emplea una computadora personal para la generación de patrones con el software MatLab, después se realiza una adquisición y procesamiento con hardware de Biopac y por último se representan los patrones en una segunda computadora por medio del software Acqknowledge.

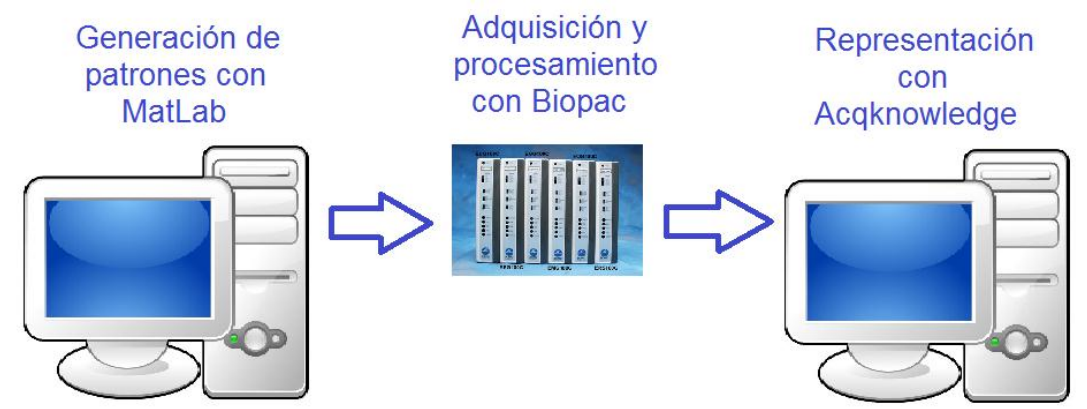

Figura 1.15. Hardware y software del método usado para el control de la respiración.

\section{I.5 Justificación}

Los dos primeros dispositivos reportados en 2001 y 2003 no son más que marcadores de tiempo para frecuencias fijas. No hay parámetro de comparación ya que no se realizaron mediciones acerca de su precisión, exactitud ni certeza. La única referencia es la comparación de métodos que se realizó en estudios piloto en el año 2012 en dónde se comprobó que el error absoluto promedio en frecuencia fue de $0.09 \mathrm{~Hz}$ con respecto al patrón de referencia calculado.

A partir de estos dos dispositivos anteriores surge la necesidad de diseñar la tercera generación que es el GPRV el cual debe poseer las características suficientes para que pueda representar los patrones de los sujetos en tiempo real con una calibración exacta y precisa y además que sea capaz de reproducir patrones respiratorios con precisión, exactitud y certeza muy cercanos al patrón de referencia construido en MatLab para que sea denominado como instrumento. 


\section{I.6 Objetivos}

1- Desarrollar un GPRV con las siguientes características:

a. Capaz de generar patrones respiratorios con frecuencias fijas y amplitudes constantes.

b. Capaz de generar patrones respiratorios con barridos de frecuencia y amplitud.

c. Que posea una entrada analógica para mostrar la respiración de los sujetos bajo prueba.

d. Que tenga más portabilidad que los dos anteriores y el método TFT.

e. El consumo de energía sea menor que el método de referencia.

2- Debe ser capaz de reproducir los siguientes patrones de respiración ${ }^{2}$ en una escala visual vertical de indicadores luminosos con una mejor precisión, exactitud y certeza que los anteriores:

a. Patrón de respiración Amplitud Constante - Frecuencia Constante (ACFC) amplitud del volumen de 1 litro, frecuencia de $0.2 \mathrm{~Hz}$ con tiempo de duración del protocolo de 1 min.

Con este patrón se observará un incremento considerable de la ASR en cada respiración.

b. Patrón de respiración Amplitud Constante - Frecuencia Variable en incremento lineal (ACFVi), amplitud del volumen de 1 litro, frecuencia de 0.15 a $0.6 \mathrm{~Hz}$ con tiempo de duración del protocolo de 1 $\min$.

Con este patrón se observará una relación inversa con respecto al aumento de frecuencia.

c. Patrón de respiración Amplitud Variable en incremento lineal Frecuencia Constante (AViFC), frecuencia de $0.2 \mathrm{~Hz}$ y amplitud variable de 0.2 a 2 litros con tiempo de duración del protocolo de 1 min.

\footnotetext{
${ }^{2}$ Estos patrones son los más utilizados en las investigaciones del Lab. de Fisiología Médica y bajo esas condiciones de frecuencia y amplitud se logra estimular con mayor impacto la rama parasimpática. 
El efecto esperado de este patrón es un aumento en la magnitud de la ASR al incremento del volumen de respiración pero aún es controversial este hecho.

3- Generar la evidencia suficiente que sustente la utilidad fisiológica del GPRV por medio de la obtención experimental de la ASR resultante de los patrones citados en el objetivo anterior ante una muestra de sujetos de experimentación.

\section{I.7 Hipótesis}

1- El control de la respiración por medio del GPRV produce patrones de respiración y ASR con error absoluto menor que los dispositivos metrónomos anteriores.

2- Los patrones de respiración y la ASR producida por el GPRV no tienen diferencias significativas con respecto del procedimiento visual de referencia con TFT. 


\section{CAPÍTULO II METODOLOGÍA}

"Ideas geniales son aquellas de las que lo único que nos sorprende es que no se nos hayan ocurrido antes"

- Noel Clarasó | España 


\section{CAPÍTULO II}

\section{METODOLOGÍA}

\section{II.1 Especificaciones de diseño del GPRV}

Para la evaluación de la ASR es indispensable estimular la rama nerviosa parasimpática por medio de la respiración en intervalos bien definidos en frecuencia [8], se han reportado ampliamente estos efectos. Estos efectos se observan en el comportamiento espectral ante una estimulación en frecuencia respiratoria de 0.2 a $0.4 \mathrm{~Hz}$. Figura 2.1.

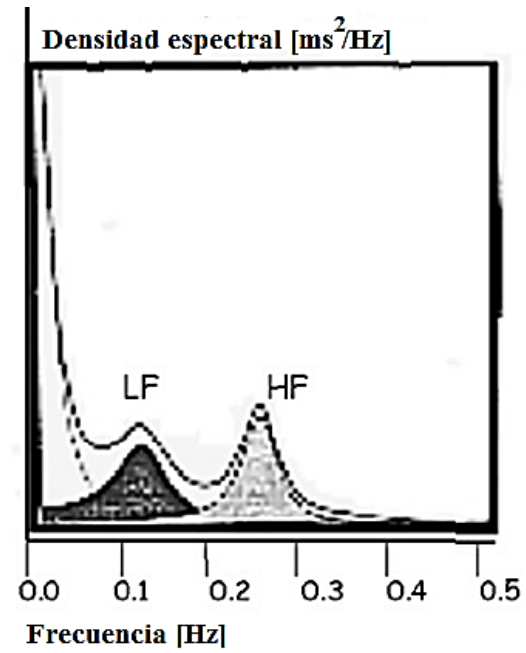

Figura 2.1. Comportamiento espectral de la VFC.

En cuanto a la amplitud los resultados siguen siendo ambiguos pero es comúnmente aceptado que la ASR se incremente en proporción a los incrementos en el volumen respiratorio. 
Por lo tanto para el diseño del GPRV se tomaron en cuenta las siguientes especificaciones:

Fisiológicas

1- Programación de patrones de respiración con frecuencias y amplitudes fijas. Rango de frecuencia de 0.1 a $0.6 \mathrm{~Hz}$, amplitud del volumen de 500 a $3500 \mathrm{ml}$.

2- Programación de patrones de respiración con frecuencias y amplitudes variables.

3- Rango de frecuencia de $0.1 \mathrm{a} 0.6 \mathrm{~Hz}$, amplitud del volumen de $500 \mathrm{a} 3500 \mathrm{ml}$.

4- Resolución en frecuencia de.01, resolución en amplitud de $50 \mathrm{ml}$.

Hardware

1- Dos sistemas embebidos basados en microcontrolador y etapa de potencia para control independiente de LED. Es indispensable que el sistema embebido posea los controladores de LED embebidos en el mismo sistema para que todo el instrumento quedé en un solo montaje.

2- Dos columnas de 70 LED cada uno. Uno de ellos es para mostrar los patrones generados por un sistema embebido. El otro para mostrar la señal de respiración proveniente del sujeto. Para tener una resolución de amplitud de $50 \mathrm{ml}$ se requieren $70 \mathrm{LED}, 3500 / 70=50 \mathrm{ml}$.

3- Entrada analógica. La entrada analógica es indispensable para mostrar la respiración de los sujetos en la segunda columna de 70 LED.

4- Control del instrumento vía remota. Para facilitar al personal que tome las muestras de los estudios.

Software

1- Programación en lenguaje de alto nivel. Facilita la programación ya que las instrucciones son más cercanas al lenguaje utilizado por el humano.

2- Licencia de uso libre. Para que el costo de todo el sistema se reduzca.

\section{II.2 Selección de la base tecnológica}

La toma de decisión para la selección se basó en estudios previos realizados en mi proyecto terminal de ingeniería [18] en donde se evaluaron microcontroladores AVR y PIC de gama baja, media y alta llegando a la conclusión de que los 
microcontroladores AVR son más modernos y cuentan con mejores recursos periféricos además de tener la ventaja de ser programados en entornos de programación de uso libre y por ello un menor costo.

En la tabla 2.1 se muestran los comparativos hechos a los sistemas embebidos con el fin de realizar la selección de la base tecnológica.

Tabla 2.1. Comparativos de cuatro sistemas embebidos basados en microcontrolador en el año 2014.

\begin{tabular}{|c|c|c|c|c|c|c|c|}
\hline & Sistema & $\begin{array}{c}\text { Gestión de } \\
\text { energía }\end{array}$ & $\begin{array}{c}\text { Controlador para } \\
\text { LED }\end{array}$ & $\begin{array}{c}\text { Costo } \\
\mathbf{\$}\end{array}$ & $\begin{array}{c}\text { Lenguaje de alto } \\
\text { nivel }\end{array}$ & IDE & Selección \\
\hline 1 & $\begin{array}{c}\text { PIC } ® 16 \mathrm{~F} 877 \mathrm{~A} \\
\mathrm{KIT}\end{array}$ & $\mathrm{Si}$ & $\mathrm{No}$ & $\begin{array}{c}\$ 7,037 \\
*\end{array}$ & $\mathrm{Si}$ & $\mathrm{Si}$ & \\
\hline 2 & $\mathrm{AVR}^{\mathrm{TM}}$-Arduino & $\mathrm{Si}$ & $\mathrm{No}$ & $\begin{array}{c}\$ 2,400 \\
* *\end{array}$ & $\mathrm{Si}$ & $\mathrm{Si}$ & \\
\hline 3 & $\begin{array}{c}\mathrm{AVR} \mathrm{R}^{\mathrm{TM}}- \\
\text { Lightuino }\end{array}$ & $\mathrm{Si}$ & $\mathrm{Si}$ & $\begin{array}{c}\$ 3,600 \\
\mathrm{n}\end{array}$ & $\mathrm{Si}$ & $\mathrm{Si}$ & $\checkmark$ \\
\hline
\end{tabular}

* El sistema embebido 1 basado en PIC tiene un compilador con costo de licencia y no se incluyen los chips controladores de LED.

** En el sistema embebido 2 basado en AVR el costo del cable de conexión y la tarjeta de expansión controladora de LED no se incluye. El compilador es GNU.

En el sistema embebido lightuino de Andrew Stone se incluyen los chips controladores de LED y el entorno de programación es GNU, compatible con arduino.

Por lo tanto la selección se realiza hacia el sistema Lightuino® de Andrew Stone, debido a que cumple con todos los requerimientos del diseño.

\section{II.2.1 Diagrama principal}

Se propuso con base en la selección del sistema embebido lightuino un diagrama general de diseño del GPRV, figura 2.2.

Encerrados con líneas punteadas se muestran los dos sistemas embebidos lightuino, donde se destaca el corazón del sistema basado en microcontroladores AVR ATMega328 con CAD de 10 bits. Dos módulos controladores de LED Micrel MM5451 con entradas de datos seriales, compatibilidad TTL y 35 salidas 
independientes por cada chip, en total 70 salidas independientes por cada sistema embebido.

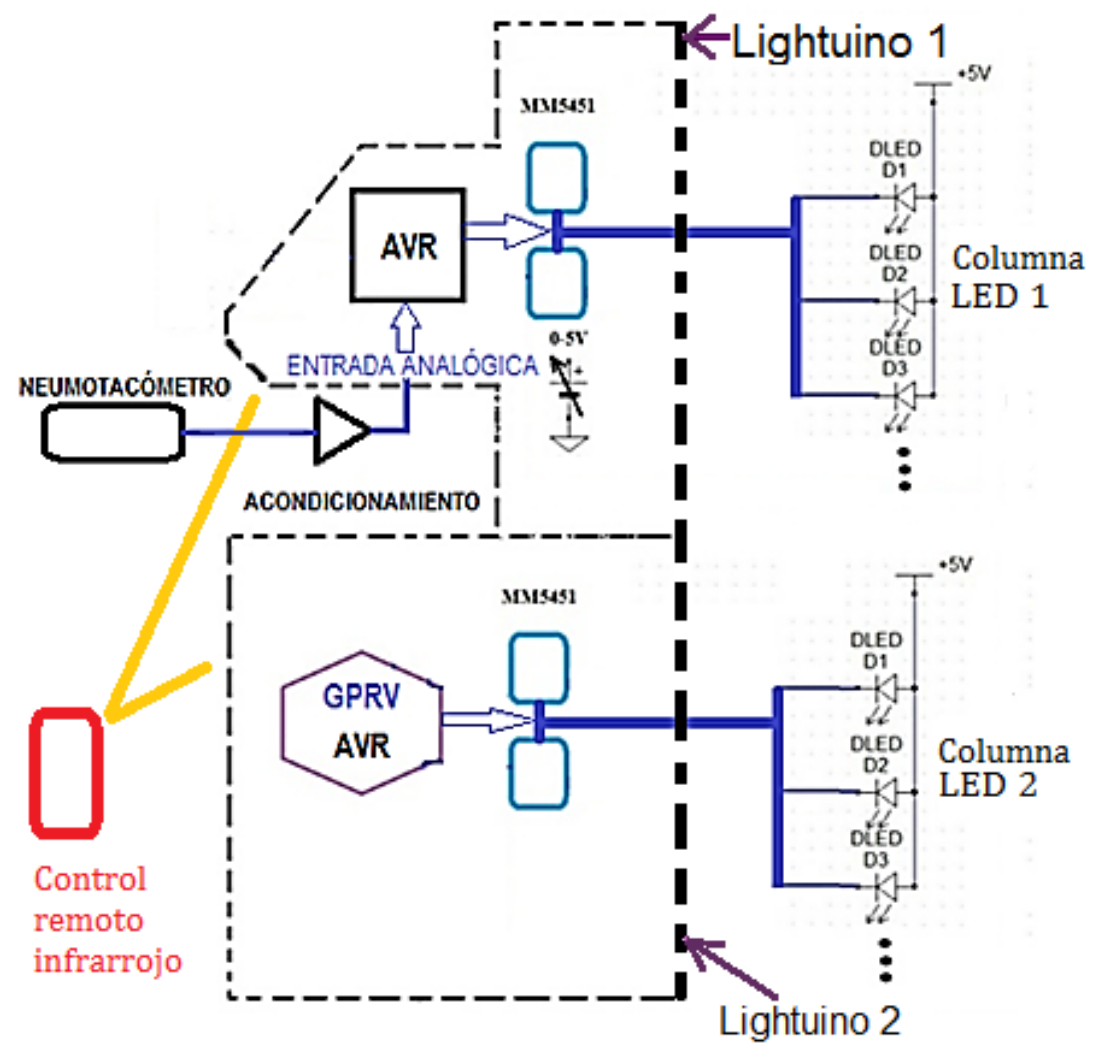

Figura 2.2. Diagrama general del GPRV.

El sistema embebido lightuino 2 se utiliza para mostrar los patrones respiratorios en la columna vertical de LED 2.

El sistema embebido lightuino 1 se utiliza exclusivamente para la entrada analógica suministrada por la circuitería del neumotacómetro la cual debe de ser acondicionada ya que la salida de voltaje lineal típica es de 0 a $1.56 \mathrm{~V}$ para 2 litros y de 0 a $0.78 \mathrm{~V}$ para 1 litro. La entrada para la adquisición del CAD del AVR requiere una entrada de 0 a $5 \mathrm{~V}$.

A cada sistema embebido se le construyó un sistema de visualización conformado por una columna vertical de 70 LED.

Aprovechando que los sistemas lightuino traen embebidos receptores infrarrojos y que se requiere que estos sean controlados remotamente se implementó un sistema de control remoto por infrarrojos, para la activación de los patrones o modo de espera. 


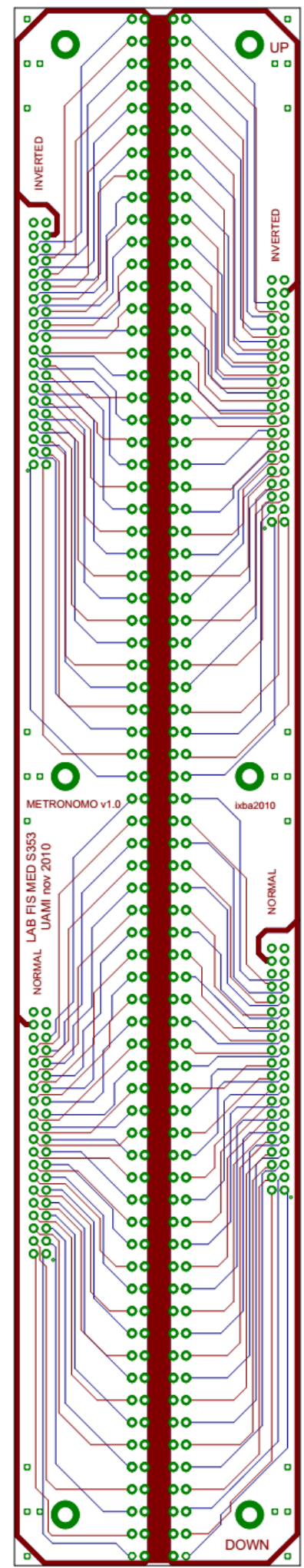

Figura 2.3. Diseño PCB Módulo visual GPRV.
Los módulos externos a los sistemas embebidos se diseñaron y construyeron de la siguiente manera:

1- Un módulo de salida: Módulo visual de dos columnas verticales dispuestas en paralelo de 70 LED, donde cada columna fue diseñada con LED de distinto color para hacer su diferenciación, el módulo visual fue fabricado en las instalaciones de la UAM-I. Se representa en una columna vertical los patrones respiratorios (LED color rojo brillante) y en la otra la respiración del sujeto (LED color verde brillante) figura 2.3.

2- Módulo de acondicionamiento del neumotacómetro: un módulo amplificador de acondicionamiento diseñado in situ para acondicionar la señal de 0 a $1.56 \mathrm{~V}$ para 2 litros a voltajes entre 0 y 5 $\mathrm{V}$ o de 0 a $0.78 \mathrm{~V}$ para 1 litro a voltajes entre 0 y $5 \mathrm{~V}$, requeridos por el CAD del AVR.

La alimentación de todo el sistema se realizó por medio de dos pilas plomo-acido de $9 \mathrm{~V}$ con esto evitamos:

1- El ruido eléctrico de $60 \mathrm{~Hz}$ y otros que pudieran introducirse provenientes de la línea de alimentación de energía.

2- Se dispone de mayor movilidad del instrumento para futuras mediciones bajo otras dinámicas de caracterización de la ASR en donde el sujeto puede estar parado, acostado o en otros ángulos, ya que la respuesta en el cambio de postura presenta grandes cambios en el análisis espectral de la frecuencia cardiaca [37], demostrado así por Pomerantz y cols. en 1985. Figura 2.4.

Se utilizan dos pilas de $9 \mathrm{~V}$ debido a que la alimentación del amplificador LM1458CN debe ser simétrica. 


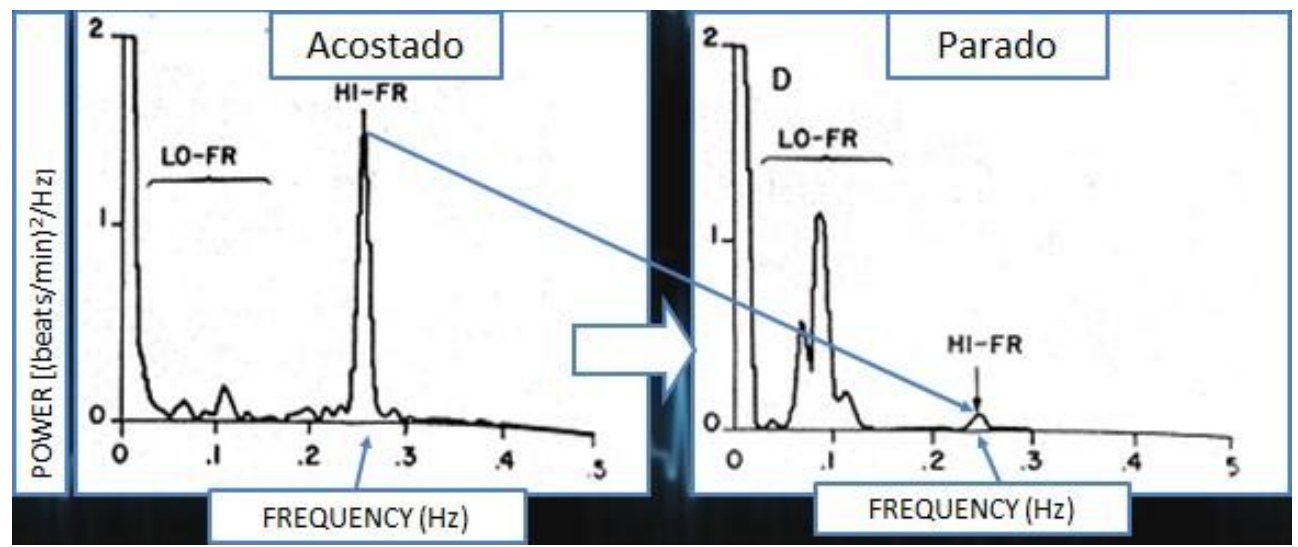

Figura 2.4. Efectos del cambio de postura en al análisis espectral de la frecuencia cardiaca [37].

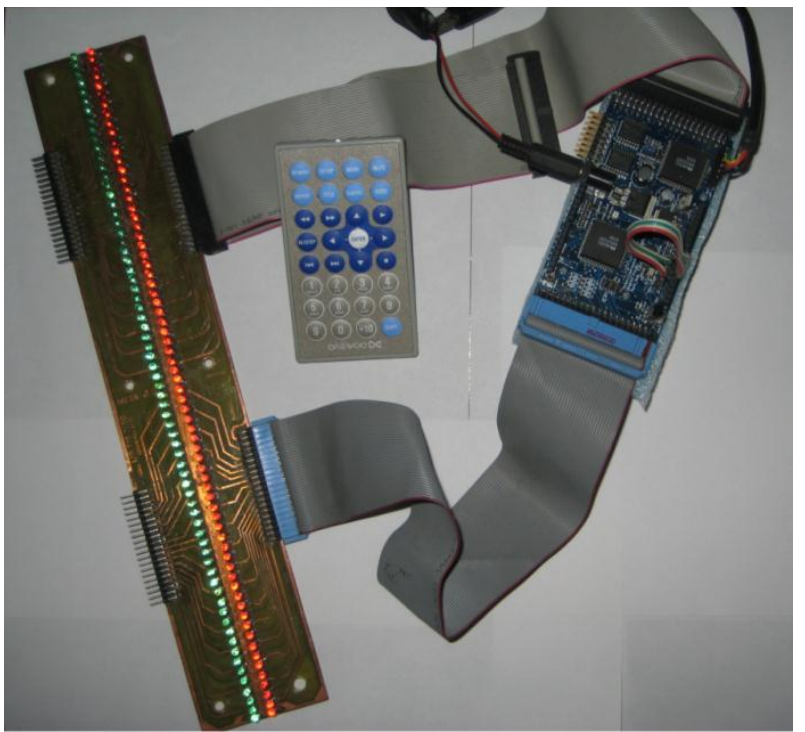

Figura 2.5. GPRV con un módulo lightuino.

La figura 2.5 muestra el GPRV con uno de los dos sistemas embebidos, el módulo de visualización de LED con sus columnas, roja para el generador de patrones y verde para la señal de respiración del sujeto, control remoto infrarrojo y conectores tipo peine de 40 conductores lo que destaca su diseño modular para su fácil integración $\quad \mathrm{y} \quad$ posterior mantenimiento.

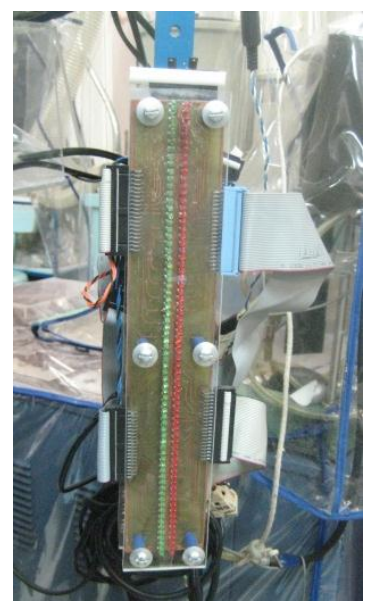

En la figura 2.6 se muestra el GPRV en su diseño completo y funcional, montado en el Laboratorio de Fisiología Médica de la Universidad Autónoma Metropolitana Unidad Iztapalapa.

Figura 2.6. GPRV montado en el Lab. de Fis. Médica. 


\section{II.2.2 Módulo visual}

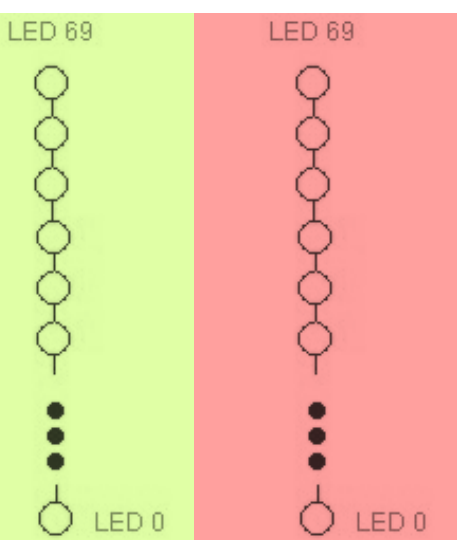

Figura 2.7. Columnas de LED.

Referente al módulo visual se seleccionaron LED's de $3 \mathrm{~mm}$ de cubierta clara que tienen mayor brillo; el hecho de elegir este tipo de cubierta fue para reducir el voltaje de alimentación y por ende su consumo de energía; LED's rojos para el patrón de respiración (columna roja) y verdes para el seguimiento analógico de la respiración de los sujetos (columna verde). Ver figura 2.7.

Se utilizaron las siguientes especificaciones para ambos vectores visuales:

Modulo visual de $31 \mathrm{~cm}$ por $5.7 \mathrm{~cm}$. El tamaño del módulo se definió bajo dos condiciones: las pantallas usadas y el campo de visión del ojo humano en condiciones normales.
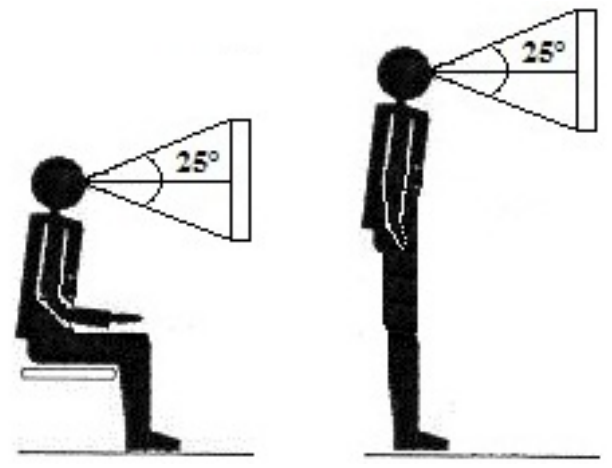

El campo visual de un ser humano es de aproximadamente $60^{\circ}$ por encima y debajo de la horizontal. [19, 20]. En nuestro modelo se considera un desplazamiento ocular de aproximadamente $25^{\circ}$ hacía arriba y hacia abajo a una distancia no mayor de $60 \mathrm{~cm}$. Ver figura 2.8.

Figura 2.8. Campo visual del GPRV.

El tamaño vertical es el que se usa en una pantalla de 26 pulgadas. Con esto se pretende evitar la fatiga en los orbitales [20]. 


\section{II.2.3 Diseño de la etapa de acondicionamiento del neumotacómetro al GPRV}

La salida de voltaje del neumotacómetro puede ajustarse desde aproximadamente 0 hasta $0.78 \mathrm{~V}$ para un rango de volumen de 1 litro. Análogamente para 2 litros el rango de voltaje es de 0 a $1.56 \mathrm{~V}$.

Debido a que la entrada del CAD del microcontrolador AVR tiene un voltaje de referencia a $5 \mathrm{~V}$ fue preciso acondicionar la señal proveniente del neumotacómetro a este valor de referencia.

Para ello fue recomendable implementar una etapa de acondicionamiento con amplificador operacional.

Las frecuencias de la señal de entrada son muy bajas menores a $1 \mathrm{~Hz}$ por lo tanto la velocidad de cambio (slew rate) no fue un factor decisivo.

El mantener por separado un acondicionamiento para el rango de volumen de 1 litro y 2 litros, fue muy importante pues es necesario cambiar entre uno y otro rango rápidamente al estar haciendo las mediciones de cada sujeto. Este factor es entonces decisivo. La tabla 2.2 muestra el resumen del diseño de la etapa de acondicionamiento del neumotacómetro para los dos rangos de experimentación utilizados.

Tabla 2.2. Diseño de la etapa de acondicionamiento del neumotacómetro.

Diseño del amplificador de acondicionamiento.

\begin{tabular}{|c|c|c|c|c|}
\hline $\begin{array}{c}\text { Rangos } \\
\text { dinámicos } \\
\text { en litros }\end{array}$ & $\begin{array}{l}\text { Rango dinámicos } \\
\text { de salida del } \\
\text { neumotacómetro. }\end{array}$ & $\begin{array}{c}\text { Ganancia } \\
\text { de } \\
\text { voltaje } \\
\text { exacta } \\
G_{v} \\
=\frac{V_{\text {smax }}}{V_{\text {emax }}}\end{array}$ & $\begin{array}{c}\text { Ganancia de } \\
\text { voltaje } \\
G_{v} \\
\text { Recomendable }\end{array}$ & $\begin{array}{c}\text { Calculo de las } \mathrm{R} \\
\text { para la ganancia } \\
\text { propuesta } \\
G_{v}=\frac{R 2}{R 1} \\
R 1=4.7 \mathrm{~K}\end{array}$ \\
\hline 0 a 1 litro & $0-0.78 \mathrm{~V}$ & 6.4 & 10 & Valores exactos \\
\hline 0 a 2 litros & $0-1.56 \mathrm{~V}$ & 3.2 & 10 & $R 2=30 K$ \\
\hline \multicolumn{4}{|c|}{$\begin{array}{l}\text { Este acondicionamiento se debe realizar ya que el voltaje de } \\
\text { referencia del CAD es de } 5 \mathrm{~V} \text {. }\end{array}$} & $\begin{array}{c}\text { Valores } \\
\text { recomendables } \\
R 2=R 3=50 K \\
\text { potenciómetro }\end{array}$ \\
\hline
\end{tabular}


Se seleccionó para este diseño el amplificador operacional LM1458CN con una configuración inversora y jumper para intercambio entre protocolos de 1 litro y 2 litros. Ver figura 2.9 selección con jumper 1 litro o 2 litros.

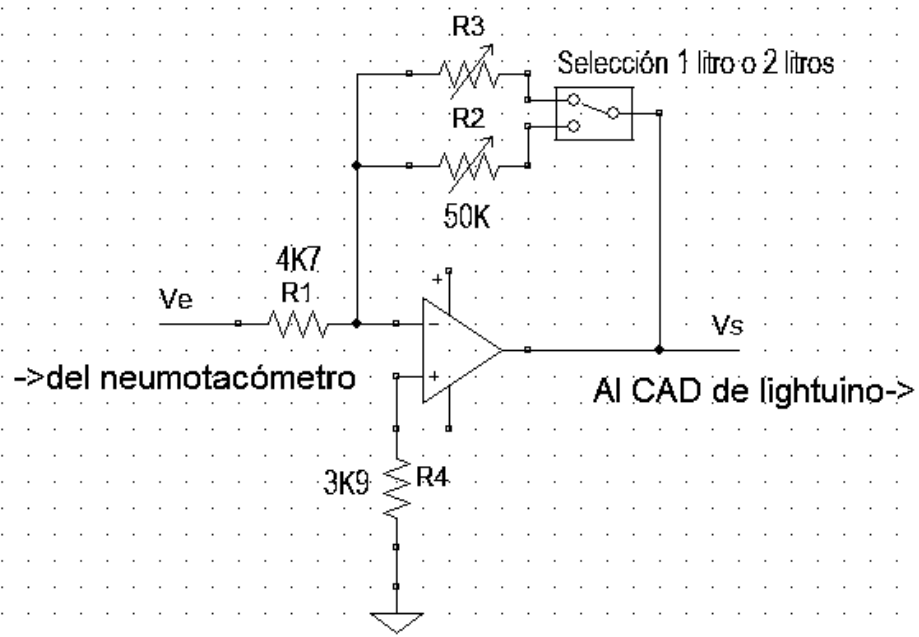

Figura 2.9. Amplificador de acondicionamiento del neumotacómetro.

Las resistencias R2 y R3 se cambiaron por dos resistores variables tipo "preset" de 50K para realizar un ajuste más exacto del valor máximo de referencia.

El valor de R4 se decidió como el promedio de los valores de R2 y R3; R4 = 3K9.

La forma de resolver el ajuste analógico de esta etapa sin la necesidad de tener la presencia de la señal del neumotacómetro fue dejar un jumper que pudiera conectar a una resistencia variable tipo "preset" entre +Vcc y -Vcc a la entrada del amplificador operacional y una vez finalizado el ajuste colocarlo en la posición de señal proveniente del neumotacómetro. Figura 2.10 preset R5. 


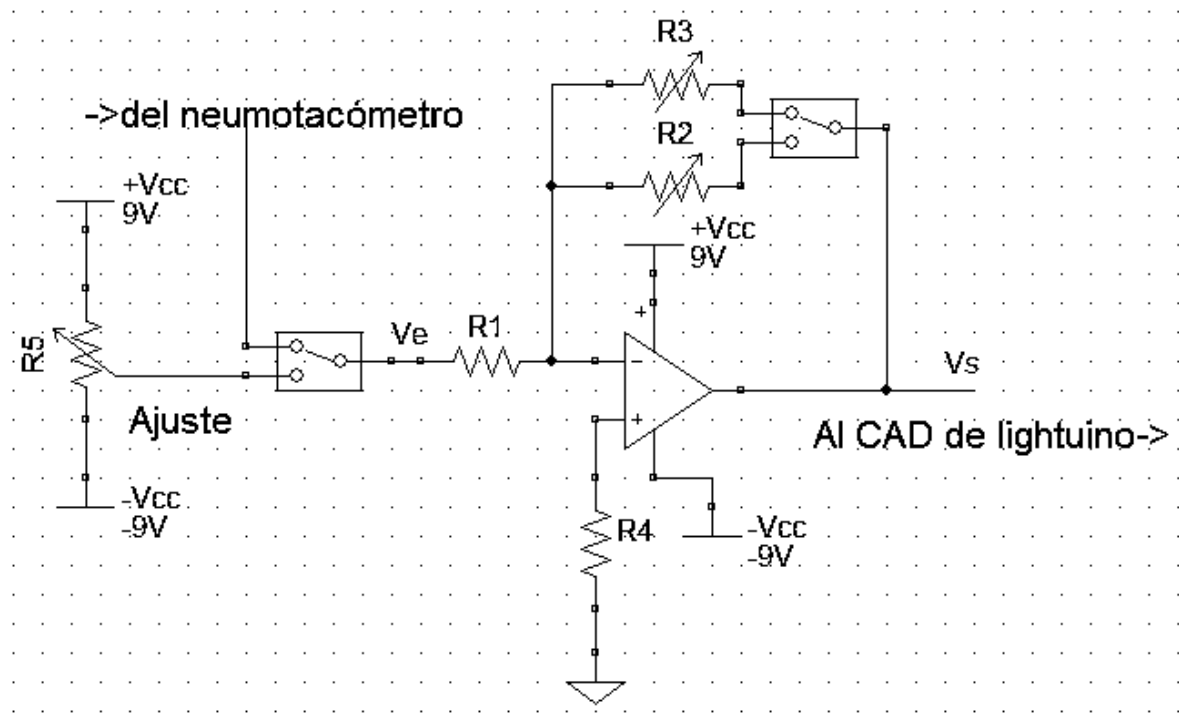

Figura 2.10. Amplificador de acondicionamiento con etapa de ajuste.

De esta manera una vez que se tenga el valor de voltaje máximo del neumotacómetro, el ajuste se puede realizar con un osciloscopio para cualquier valor de amplitud del volumen respiratorio.

\section{II.2.4 Procesamiento de la señal en el sistema embebido}

Para este fin se utilizó el CAD de 10 bits embebido dentro del microcontrolador AVR® de la tarjeta lightuino ${ }^{\mathrm{TM}}$.

El código para realizar esta acción se muestra en el anexo I sección 5.

El código es el encargado de entregar una salida binaria que es mapeada a la columna izquierda de LED (color verde) para la señal del neumotacómetro proveniente de la respiración del sujeto.

\section{II.3 Ajuste de los parámetros fisiológicos al GPRV}

Para el ajuste de parámetros fisiológicos al GPRV se consideraron dos características importantes: la frecuencia y la amplitud del volumen respiratorio (AVOR). Para nuestro modelo los cambios en volumen de aire aspirado representan cambios en amplitud en la columna de indicadores luminosos. Ver figura 2.11. 


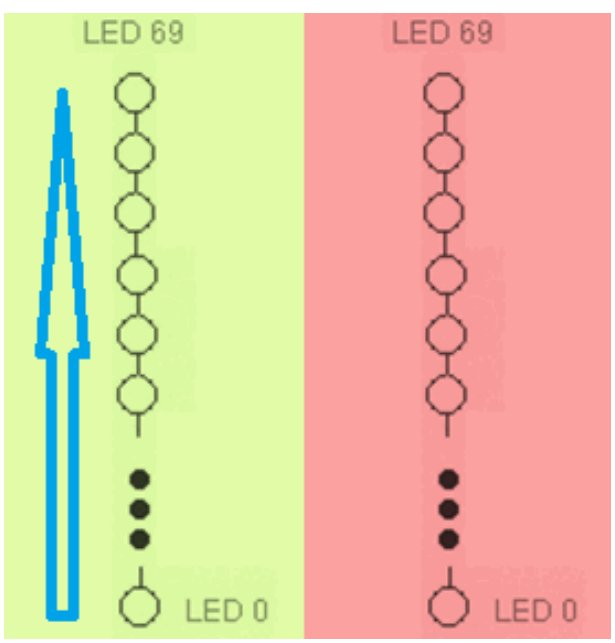

La figura 2.11 muestra que a cada incremento del volumen respiratorio se encienden mayor cantidad de LED's. De tal manera que cuando todos los LED's se encuentran encendidos esto representa un volumen de respiración equivalente a 1 o 2 litros. Cada LED mapea el volumen respiratorio de acuerdo al voltaje del neumotacómetro.

Figura 2.11. Mapeo del volumen respiratorio a la columna de LED.

\section{II.3.1 Ajuste de las funciones programadas del parámetro fisiológico frecuencia respiratoria}

La frecuencia de la respiración espontánea en sujetos sanos es de aproximadamente 12 respiraciones por minuto (12 rpm) [21].

De esta manera se establecieron dos supuestos:

1- Todas las respiraciones presentan el mismo periodo.

2- El tiempo de inhalación es igual al tiempo de exhalación.

Análisis:

Hay 12 respiraciones $(r)$ en $60 s$.

El factor de conversión (ecuación 2.1) es:

$12 r=60 s$

El tiempo de una respiración, es decir un ciclo (ecuación 2.2) es:

$$
t_{r}=1 r \frac{60 s}{12 r}=5 s
$$


El tiempo de inhalación es igual al tiempo de exhalación (ecuación 2.3)

$$
\therefore t_{\text {inspiración }}=t_{\text {espiración }}=t_{r / 2}=2.5 \mathrm{~s}-\cdots-\cdots---{ }^{2.3}
$$

Así se estableció el tiempo base para el patrón en la columna roja.

El tiempo de subida y de bajada es $2.5 \mathrm{~s}$ desde el LED 1 hasta el 70 en condiciones normales de respiración.

Ahora es necesario obtener el tiempo de encendido de cada LED en la columna roja:

Si encienden en el vector (columna) 70 LED's cada $2.5 s$.

Entonces el factor de conversión (ecuación 2.4) es:

$$
70 L E D=2.5 s
$$

$\therefore$ el tiempo de encendido por LED (ecuación 2.2.4-5) es:

$$
t_{L E D}=1 L E D \frac{t_{r} / 2}{70 L E D}----------2.5
$$

Sustituyendo los valores en la ecuación 2.5:

$$
t_{L E D}=1 L E D \frac{2.5 \mathrm{~s}}{70 \text { LED }}=.035 \overline{714285} \mathrm{~s}=35 . \overline{714285} \mathrm{~ms}
$$

Este valor $\left(t_{L E D}\right)$ resultado de la ecuación 2.5 es el retraso en milisegundos (ms) que debe llevar la programación para cada LED en la función de retraso "delay()" para este caso en particular.

La entrada de usuario para el GPRV se definió en Hertz ya que es común referirse a respuestas en frecuencia cuando se realiza el análisis espectral de la ASR. Se ha encontrado evidencia de una banda de frecuencias comprendidas entre $0.15 \mathrm{y}$ 
$0.40 \mathrm{~Hz}$, figura 2.12. A esta banda se le atribuye la estimulación sobre la rama parasimpática o vagal por medio de los movimientos respiratorios [22].

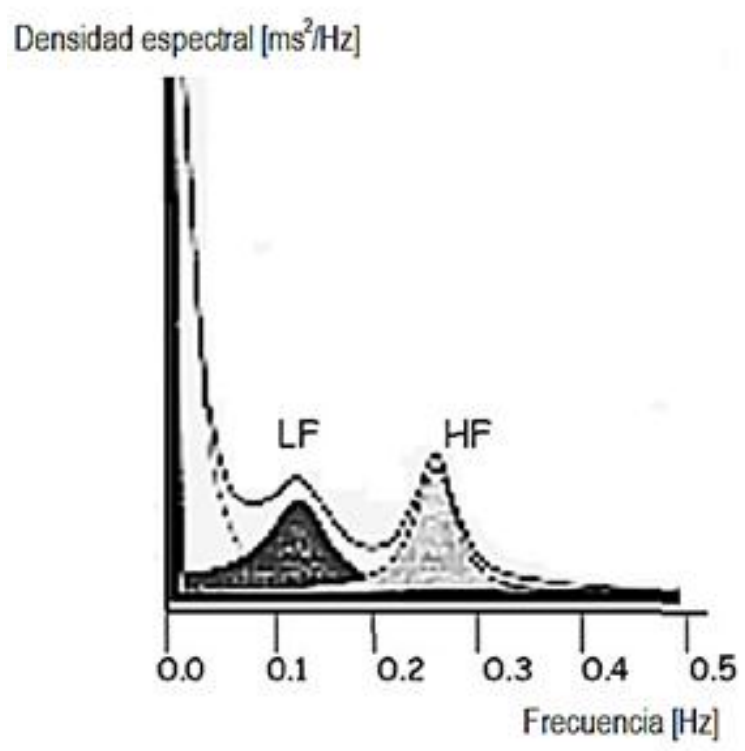

Figura 2.12. Comportamiento espectral de la ASR.

LF (Low Frequency : Baja Frecuencia) -> 0.04-0.15 Hz (Simpático-Vagal); HF(High Frequency : Alta Frecuencia) $->$ 0.15-0.50 Hz (Vagal-Respiratorio).

Se encontró una relación para el parámetro de entrada de usuario en $\mathrm{Hz}$ y el parámetro de la función delay() en ms, el análisis se describe a continuación.

Cuando se mide la cantidad de respiraciones debido a su larga duración se emplea el minuto como referencia.

La frecuencia en Hertz es el reciproco de este número de respiraciones (Nrpm) con su correspondiente factor de conversión de minutos a segundos. Ver ecuación 2.6.

$$
\begin{aligned}
& f=N r p m / 60 s \\
& \therefore t=60 \mathrm{~s} / \mathrm{Nrpm}
\end{aligned}
$$

En la ecuación 2.3 se establece el supuesto de que el tiempo de inhalación o subida es igual al tiempo de exhalación o bajada. Es decir, el tiempo que toma una respiración dividido entre dos. Ver ecuación 2.8. 


$$
\frac{t}{2}=t_{1 / 2}=30 \mathrm{~s} / \mathrm{Nrpm}---------2.8
$$

Análogamente:

$$
2 f=f_{1 / 2}=N r p m / 30 s------2.9
$$

Generalizamos la ecuación $2.5\left(t_{L E D}\right)$ en la ecuación 2.10.

$$
t_{L E D}=\frac{t_{1} / 2}{V_{L E D}}
$$

Donde $V_{L E D}$ es el tamaño del vector o columna de LED's, siendo una constante con valor de 70 .

Al sustituir el valor de $V_{L E D}$ y la ecuación 2.8 en la ecuación 2.10, se consigue la ecuación 2.11 para cualquier valor de entrada en respiraciones por minuto (Nrpm):

$$
t_{L E D}=\frac{30 \mathrm{~s}}{\operatorname{Nrpm}(70)}
$$

La entrada de usuario debe ser en Hertz, entonces de la ecuación 2.9 se resuelve para Nrpm:

$$
N r p m=2 f(30 \mathrm{~s})-------------2.12
$$

Al sustituir la ecuación 2.12 en la ecuación 2.11, simplificar y convertir el tiempo en segundos a milisegundos se consigue la ecuación 2.13 para cualquier valor de entrada en Hertz:

$$
t_{L E D}=\frac{1}{f} c
$$

Donde $c=$ constante adimensional de ajuste para la función delay $=\frac{50}{7}$

La ecuación 2.13 se usó entonces para realizar la programación de la entrada de usuario en Hz. Ver anexo I para más detalles.

Con esta ecuación se cubre el rango dinámico de 0.1 a $0.6 \mathrm{~Hz}$ necesario para ambas bandas de frecuencias citadas en la figura 2.12 . 
En la tabla 2.3 se muestra en su columna Nrpm los valores de interés referentes a la cantidad de respiraciones por minuto.

Tabla 2.3. Frecuencias a programar en el GPRV.

\begin{tabular}{|c|c|}
\hline $\begin{array}{c}\text { Respiraciones por minuto } \\
\text { Nrpm }\end{array}$ & $\begin{array}{c}\text { Frecuencia } \\
\text { Hz }\end{array}$ \\
\hline 9 & 0.15 \\
\hline 10 & 0.17 \\
\hline 11 & 0.18 \\
\hline 12 & 0.20 \\
\hline 13 & 0.22 \\
\hline 14 & 0.23 \\
\hline 15 & 0.25 \\
\hline 16 & 0.27 \\
\hline 17 & 0.28 \\
\hline 18 & 0.30 \\
\hline 19 & 0.32 \\
\hline 20 & 0.33 \\
\hline 21 & 0.35 \\
\hline 22 & 0.37 \\
\hline 23 & 0.38 \\
\hline 24 & 0.40 \\
\hline 25 & 0.42 \\
\hline 26 & 0.43 \\
\hline 27 & 0.45 \\
\hline 28 & 0.47 \\
\hline 29 & 0.48 \\
\hline 30 & 0.5 \\
\hline 31 & 0.52 \\
\hline 32 & 0.53 \\
\hline 33 & 0.55 \\
\hline 34 & 0.57 \\
\hline 35 & 0.58 \\
\hline 36 & 0.6 \\
\hline
\end{tabular}

La barra vertical en verde muestra el rango de valores considerados como normales en un adulto. 
Con amarillo se muestra la frecuencia aproximada donde existe un máximo en la amplitud de la densidad de energía para las frecuencias altas. Y las frecuencias en azul representan el rango donde se presentan los mayores efectos fisiológicos de la ASR. Este conjunto de frecuencias se tomó como rango dinámico para el GPRV.

\section{II.3.1.1 Calibración del GPRV en frecuencia}

Para realizar la calibración del GPRV en frecuencia se utilizó como instrumento de medición un osciloscopio Tektronix modelo TDS1001 y la interfaz del monitor serial de arduino. El osciloscopio se conectó en el último LED (LED 70) de la columna que muestra los patrones respiratorios, con esto se obtienen los tiempos de la mitad del periodo de cada respiración, figura 2.13a y 2.13b. En el caso del monitor arduino se utilizaron funciones ya definidas que contabilizan el tiempo desde un punto inicial en el código que se ejecuta hasta un punto final, para realizar el comparativo entre las mediciones del osciloscopio y las del monitor de arduino se tomó el tiempo de un periodo, es decir desde que inicia la secuencia de encendido en el primer LED o LED inferior de la columna roja hasta que termina la secuencia de encendido en el último LED o LED superior de la columna roja.

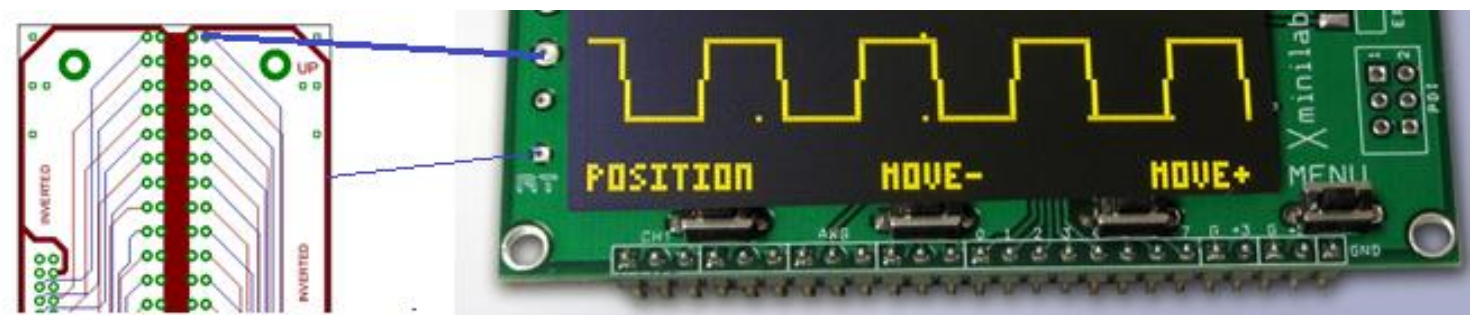

Figura 2.13a Conexión del osciloscopio al GPRV.

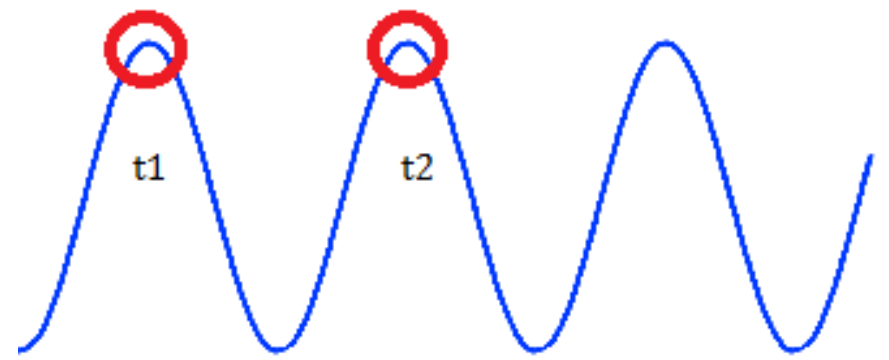

Figura 2.13b tiempos tomados con el osciloscopio del patrón del GPRV.

De esta manera tomando la diferencia de $\mathrm{t} 1 \mathrm{y} \mathrm{t} 2$, figura $2.13 \mathrm{~b}$, se obtiene el periodo con el cuál es posible calcular la frecuencia. 
Para cada frecuencia del rango dinámico $(0.15$ a $0.4 \mathrm{~Hz})$, tabla 2.4 , se tomaron 13 mediciones, a partir de estas mediciones se obtuvieron los valores promedio y se calcularon los errores absolutos, tabla 2.5.

Tabla 2.4. Rango dinámico del GPRV.

\begin{tabular}{|c|c|}
\hline $\begin{array}{c}\text { Respiraciones por minuto } \\
\text { Nrpm }\end{array}$ & $\begin{array}{c}\text { Frecuencia } \\
\text { Hz }\end{array}$ \\
\hline 9 & 0.15 \\
\hline 10 & 0.17 \\
\hline 11 & 0.18 \\
\hline 12 & 0.20 \\
\hline 13 & 0.22 \\
\hline 14 & 0.23 \\
\hline 15 & 0.25 \\
\hline 16 & 0.27 \\
\hline 17 & 0.28 \\
\hline 18 & 0.30 \\
\hline 19 & 0.32 \\
\hline 20 & 0.33 \\
\hline 21 & 0.35 \\
\hline 22 & 0.37 \\
\hline 23 & 0.38 \\
\hline 24 & 0.40 \\
\hline
\end{tabular}

Tabla 2.5. Cálculo de los errores de los valores promedio de cada frecuencia.

\begin{tabular}{|c|c|c|c|c|}
\hline $\begin{array}{l}\text { Frecuencia [Hz] } \\
\text { calculada }\end{array}$ & $\begin{array}{c}\text { Frecuencia } \\
\text { [Hz] medida } \\
\text { con } \\
\text { Osciloscopio } \\
\text { Valores } \\
\text { promedio }\end{array}$ & $\begin{array}{c}\text { Frecuencia } \\
\text { [Hz] medida } \\
\text { por } \\
\text { Software } \\
\text { Valores } \\
\text { promedio }\end{array}$ & & \\
\hline$X n$ & Yno & Yns & $\begin{array}{l}\text { error abs } \\
\text { |Yno-Xn| }\end{array}$ & $\begin{array}{l}\text { error abs } \\
\mid \text { Yns-Xn| }\end{array}$ \\
\hline 0.150 & 0.152 & 0.151 & 0.002 & 0.001 \\
\hline 0.167 & 0.167 & 0.168 & 0.000 & 0.002 \\
\hline 0.183 & 0.180 & 0.181 & 0.003 & 0.001 \\
\hline 0.200 & 0.200 & 0.201 & 0.000 & 0.001 \\
\hline 0.217 & 0.219 & 0.221 & 0.002 & 0.002 \\
\hline 0.233 & 0.226 & 0.227 & 0.007 & 0.001 \\
\hline 0.250 & 0.249 & 0.251 & 0.001 & 0.002 \\
\hline 0.267 & 0.267 & 0.271 & 0.000 & 0.004 \\
\hline 0.283 & 0.283 & 0.281 & 0.000 & 0.002 \\
\hline 0.300 & 0.300 & 0.292 & 0.000 & 0.008 \\
\hline 0.317 & 0.317 & 0.318 & 0.000 & 0.001 \\
\hline 0.333 & 0.333 & 0.333 & 0.000 & 0.000 \\
\hline 0.350 & 0.350 & 0.351 & 0.000 & 0.001 \\
\hline 0.367 & 0.367 & 0.368 & 0.000 & 0.001 \\
\hline 0.383 & 0.383 & 0.378 & 0.000 & 0.005 \\
\hline 0.400 & 0.400 & 0.387 & 0.000 & 0.013 \\
\hline
\end{tabular}


Observando los errores absolutos se procedió a la calibración para cada frecuencia hasta tener el mejor valor, es decir el valor con menos diferencia entre el patrón de frecuencia calculado y la medición realizada con el osciloscopio y el monitor de arduino. Este proceso se realizó midiendo los tiempos de ejecución de las instrucciones y optimizando el código del programa, y en algunos casos colocando retrasos en puntos específicos del código.

En la figura 2.14 se muestra la recta referente al patrón Xn en color azul con rombos del mismo color en las frecuencias deseadas. Los cuadrados en color rojo Yno referentes a las mediciones hechas por el osciloscopio después de la calibración y su recta de ajuste por mínimos cuadrados con el mismo color. Se muestra también con puntos color verde Yns las mediciones hechas por el monitor serial de arduino después de la calibración y su respectiva recta de ajuste.

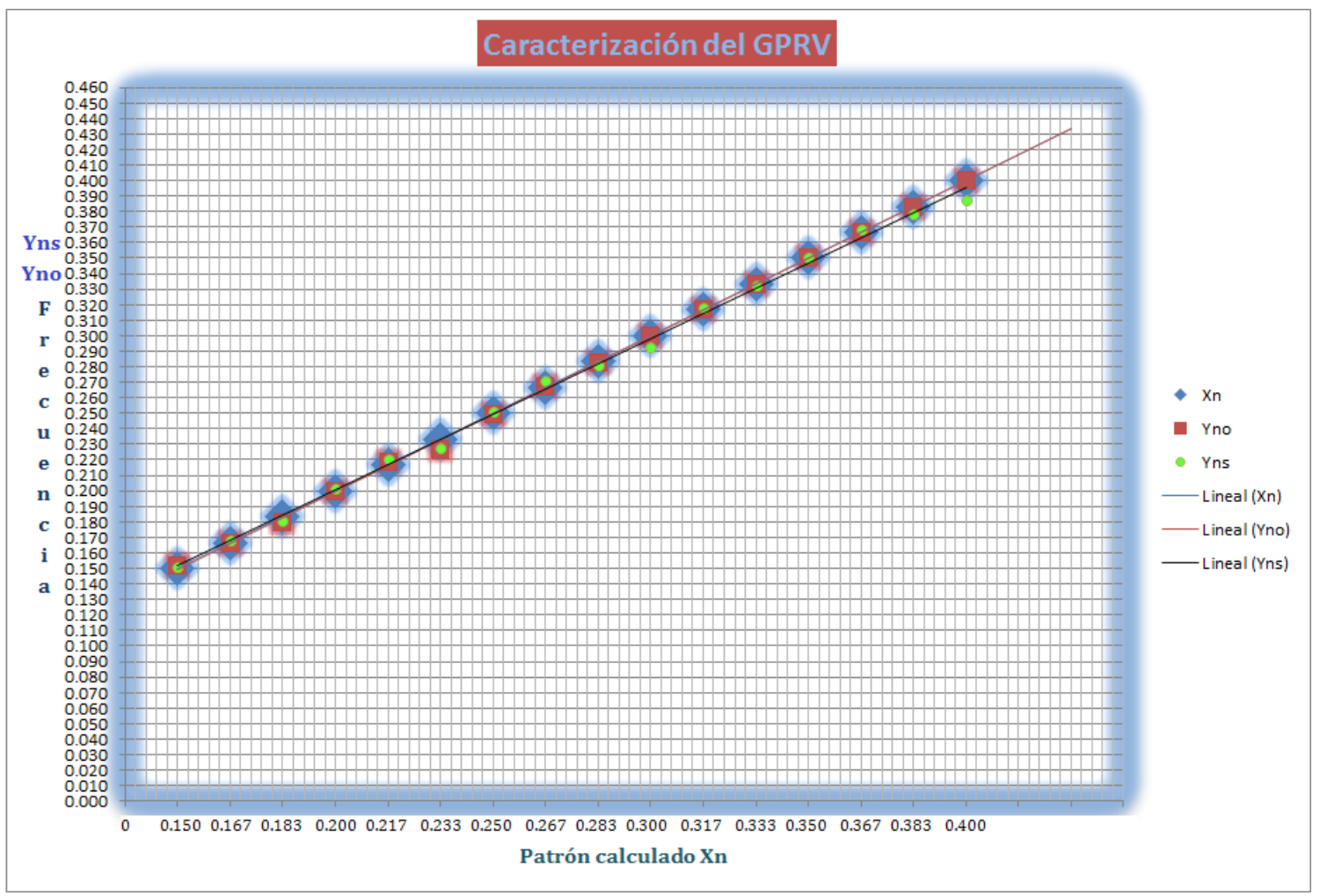

Figura 2.14 Patrón Xn lineal, tendencia y ajuste Yno (mediciones del osciloscopio) y tendencia y ajuste Yns (mediciones por monitor arduino) después de la calibración del GPRV.

Se observa que las mediciones hechas por el monitor serial de arduino son confiables apenas 0.002 milésimas de separación con respecto al error absoluto promedio de las mediciones hechas por el osciloscopio. También se observa una 
ligera tendencia a separarse de los valores del patrón Xn conforme se incrementa la frecuencia, sin embargo para estás frecuencias tan bajas no es significativo.

\section{II.3.2 Ajuste de las funciones programadas del parámetro fisiológico amplitud del volumen respiratorio}

El siguiente objetivo referente al ajuste del parámetro fisiológico AVOR (amplitud del volumen respiratorio) se basó en la teoría descrita por Guyton Arthur C. y Hall John E. en su libro Fisiología Médica 11ae ed. Unidad VII, capítulo 37 [21], y en un estudio piloto previo realizado en el Laboratorio de Fisiología Médica de la UAM Iztapalapa.

La amplitud del volumen de la respiración humana se determina por medio de un método denominado espirometría. Este método indica los cambios en amplitud del volumen pulmonar por medio de un espirograma. [21]

La teoría define los cambios de la amplitud del volumen respiratorio en cuatro volúmenes y cuatro capacidades. Ver figura 2.15.

En mujeres los volúmenes y capacidades son menores en un 20 a $25 \%$ que los hombres.

En el estudio piloto realizado en el Laboratorio se observaron máximos entre 1,500 $\mathrm{ml}$ y $2,000 \mathrm{ml}$ para varones y 1,000 a 1,500 ml en mujeres.

La información extraída del libro de Fisiología Médica (Guyton y Hall) y las observaciones del estudio previo en laboratorio se tomaron para decidir el rango de la amplitud del volumen respiratorio (AVOR).

El rango del GPRV fue de 0 a 3,500 $\mathrm{ml}$ a partir del volumen corriente qué es de aproximadamente $500 \mathrm{ml}$. Ver figura 2.15. 


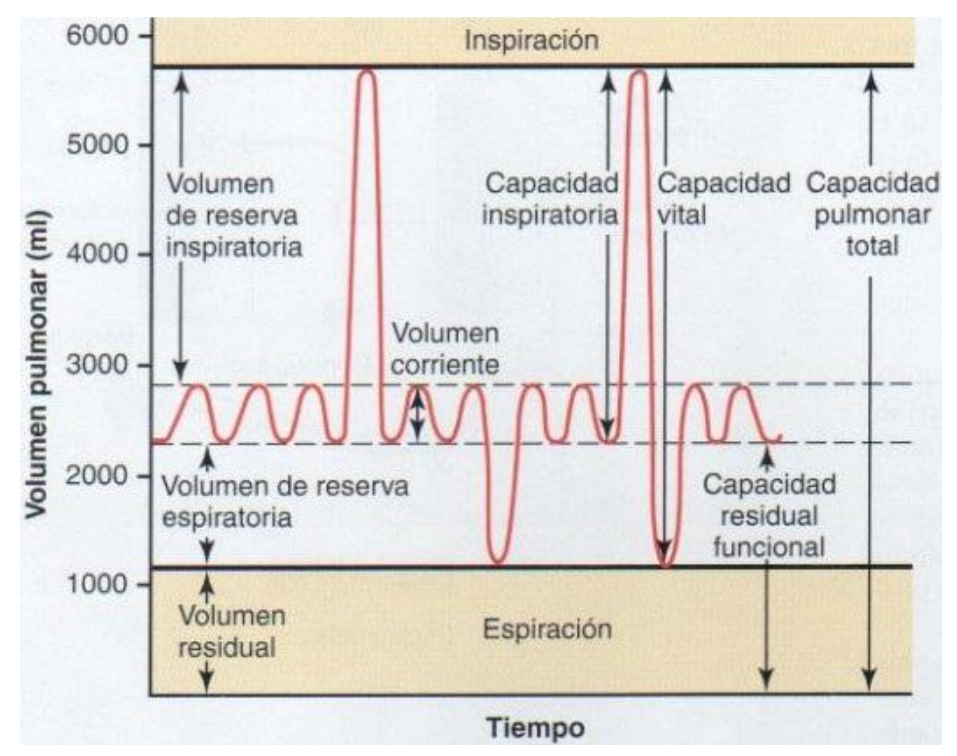

Figura 2.15. Volúmenes y capacidades pulmonares (Fisiología Médica, Guyton, Hall).

La resolución (res) del metrónomo es entonces de $50 \mathrm{ml}$ por LED como lo indica la ecuación 2.14.

$$
\begin{aligned}
& \qquad \text { res }=\frac{C I}{V_{L E D}}+\cdots \\
& C I=\text { capacidad inspiratoria }=3,500 \mathrm{ml} \\
& V_{L E D}=70 \mathrm{LED}
\end{aligned}
$$

Sustituyendo los valores en la ecuación 2.14:

$$
r e s=\frac{3,500 \mathrm{ml}}{70 \mathrm{LED}}=50 \mathrm{ml} / \mathrm{LED}
$$

Debido a que se trabajó con amplitudes diferentes es necesario resolver la ecuación de tiempo en términos de la amplitud ya que la velocidad de movimiento (encendido de los LED en la columna roja) debe ajustarse según la amplitud seleccionad, para más detalles ver anexo II. Entonces la ecuación 2.13 se resuelve para la amplitud, ésta queda en términos de la frecuencia y la amplitud:

$$
\begin{aligned}
& t_{L E D}=\frac{1}{f} c=-\cdot-\cdot---- \\
& c=\frac{1}{2\left(V_{L E D}\right)} 1000=\frac{500}{V_{L E D}}
\end{aligned}
$$


Por conveniencia se redefinió la constante $V_{L E D}$ que ahora en el caso de AVOR variable, $V_{L E D}$ se convierte en una variable, renombrada como: $A_{L E D}$ (Amplitud del volumen respiratorio por $L E D$ ) y $c_{1}$ es la nueva constante de ajuste para la ecuación 2.13.

$$
\begin{aligned}
& V_{L E D}=A_{L E D}=\text { amplitud de la columna de } L E D^{\prime} s \\
& c_{1}=500 \\
& \therefore t_{L E D}=\frac{1}{f}\left(\frac{C_{1}}{A_{L E D}}\right)-------------2.15
\end{aligned}
$$

De esta manera la ecuación 2.15 es fundamental en la programación del GPRV.

\section{II.3.2.1 Calibración del parámetro amplitud del volumen respiratorio}

En el caso de la calibración para la columna roja esta se realizó con la implementación de la ecuación 2.15 en el código del programa para generar los patrones de amplitud del volumen en sus dos modalidades: amplitud del volumen constante y amplitud del volumen variable en incremento lineal, ver figura $2.16 \mathrm{y}$ 2.17 .

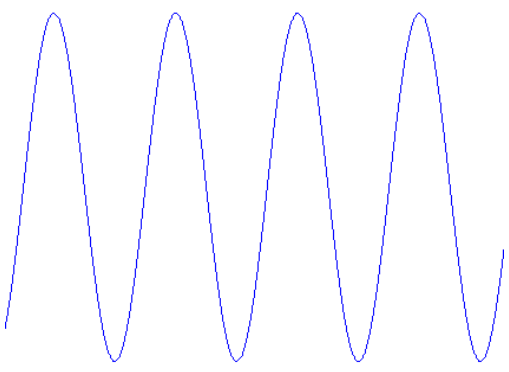

Figura 2.16. Amplitud del volumen constante.

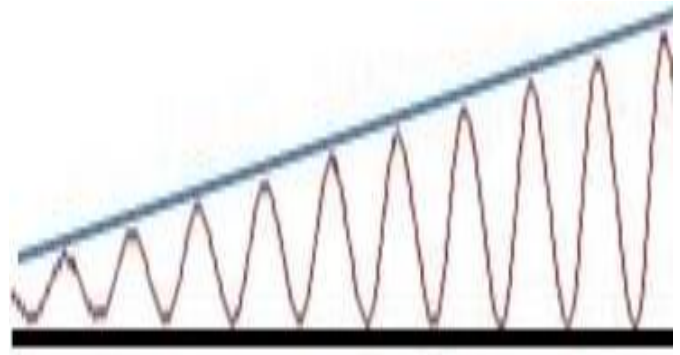

Figura 2.17. Amplitud del volumen variable en incremento lineal. 
Para calibración de la entrada analógica se siguió el procedimiento siguiente:

1- Se calibra la salida del neumotacómetro con un patrón de volumen de aire a 1 litro para voltajes de 0 a $0.78 \mathrm{~V}$, con cambios lineales.

a. Se usa el hardware de biopac y su software de adquisición Acqknowledge.

2- Se calibra la salida del neumotacómetro con un patrón de volumen de aire a 2 litros para voltajes entre 0 a $1.56 \mathrm{~V}$.

a. Se usa el hardware de biopac y su software de adquisición Acqknowledge.

3- Se calibra el canal analógico del sistema embebido lightuino con un voltaje de entrada de $0.78 \mathrm{~V}$ para el caso de 1 litro por medio del potenciómetro de ajuste R5 mostrado en la figura 2.10. Con el potenciómetro R2 se ajusta la ganancia hasta obtener $5 \mathrm{~V}$ a la salida del módulo de acondicionamiento. Estos 5V son los requeridos como voltaje de referencia del CAD del AVR.

a. Se usa como instrumento de medición un osciloscopio Tektronix modelo TDS1001 con uno de sus canales conectado a la entrada del circuito de acondicionamiento (nodo Ve) y el segundo canal conectado a la salida de la etapa de acondicionamiento nodo Vs, mostrados en la figura 2.9.

4- Se calibra el canal analógico del sistema embebido lightuino con un voltaje de entrada de $1.56 \mathrm{~V}$ para el caso de 2 litros por medio del potenciómetro de ajuste R5 mostrado en la figura 2.10. Con el potenciómetro R3 se ajusta la ganancia hasta obtener $5 \mathrm{~V}$ a la salida del módulo de acondicionamiento. Estos 5V son los requeridos como voltaje de referencia del CAD del AVR.

a. Se usa como instrumento de medición un osciloscopio Tektronix modelo TDS1001 con uno de sus canales conectado a la entrada del circuito de acondicionamiento (nodo Ve) y el segundo canal conectado a la salida de la etapa de acondicionamiento nodo Vs, mostrados en la figura 2.10. 


\section{II.4 Medición de energía consumida}

Se realizaron mediciones del consumo de corriente en los tres patrones respiratorios durante el tiempo en que todos los LED del sistema estaban encendidos, con un promedio de $1.94 \mathrm{~A}$ de todas las mediciones, por lo tanto la potencia consumida aproximada es de:

$$
P_{c}=9 \mathrm{~V}(1.94 \mathrm{~A})=17.46 \mathrm{~W}
$$

\section{II.5 Patrones de respiración}

\section{II.5.1 Patrón de respiración Amplitud Constante, Frecuencia Constante (ACFC)}

Este patrón es el más simple y consiste en establecer un valor de amplitud para el volumen de la respiración entre 500 y $3500 \mathrm{ml}$; se parte de 500 ya que el volumen corriente es de aproximadamente $500 \mathrm{ml}$; un valor de frecuencia que se encuentre entre 0.14 y $0.60 \mathrm{~Hz}$ que equivale a un rango entre 9 y $36 \mathrm{rpm}$ y un valor de duración en segundos. Figura 2.18.

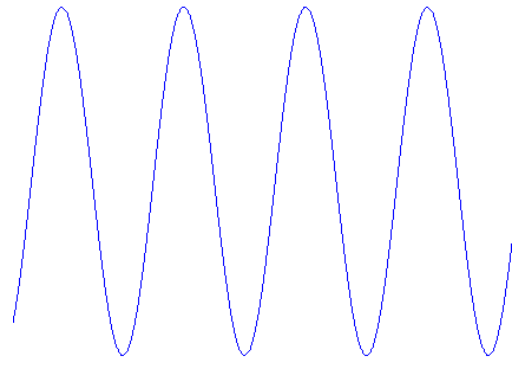

Figura 2.18. Patrón ACFC.

Los parámetros a programar son:

1- Amplitud (A) en ml.

2- Frecuencia (F) en Hz.

3- Tiempo (T) en min.

\section{II.5.2 Patrón de respiración Amplitud Variable, Frecuencia Constante (AVFC)}

En este patrón existen dos casos:

a) Amplitud variable en incremento, frecuencia constante (AViFC) ver figura 2.19.

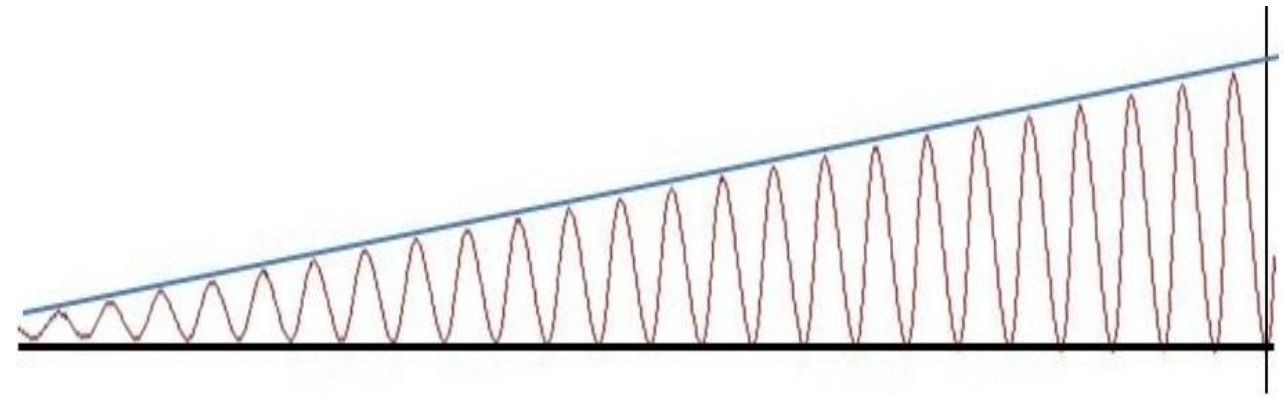

Figura 2.19. Patrón AViFC. 
b) Amplitud variable en incremento, frecuencia constante (AViFC) ver figura 2.20 .

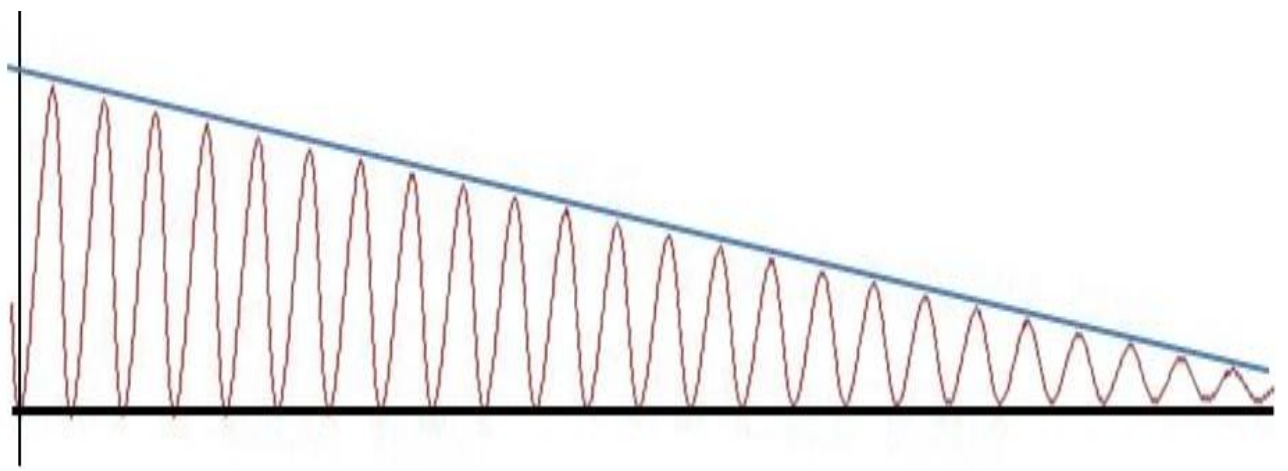

Figura 2.20. Patrón AVdFC.

Los parámetros a programar son:

1- Amplitud (A) en ml.

2- Frecuencia (F) en Hz.

3- Tiempo (T) en min.

Ver anexo I para más detalles sobre el diseño y programación.

\section{II.5.3 Patrón Amplitud Constante, Frecuencia Variable (ACFV)}

En este patrón existen dos casos:

a) Amplitud constante, frecuencia variable en incremento (ACFVi), figura 2.21.

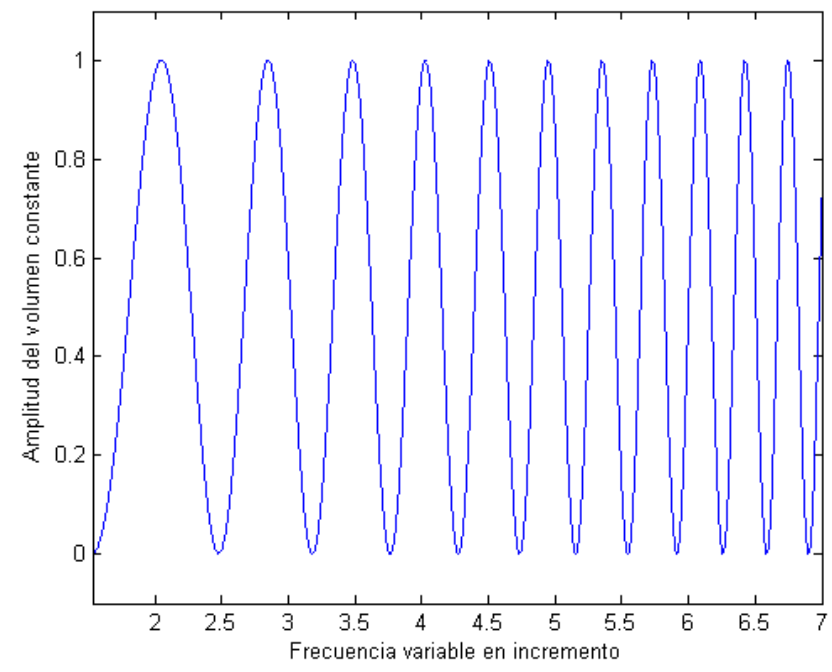

Figura 2.21. Patrón ACFVi. 
b) Amplitud constante, frecuencia variable en decremento (ACFVd), figura 2.22 .

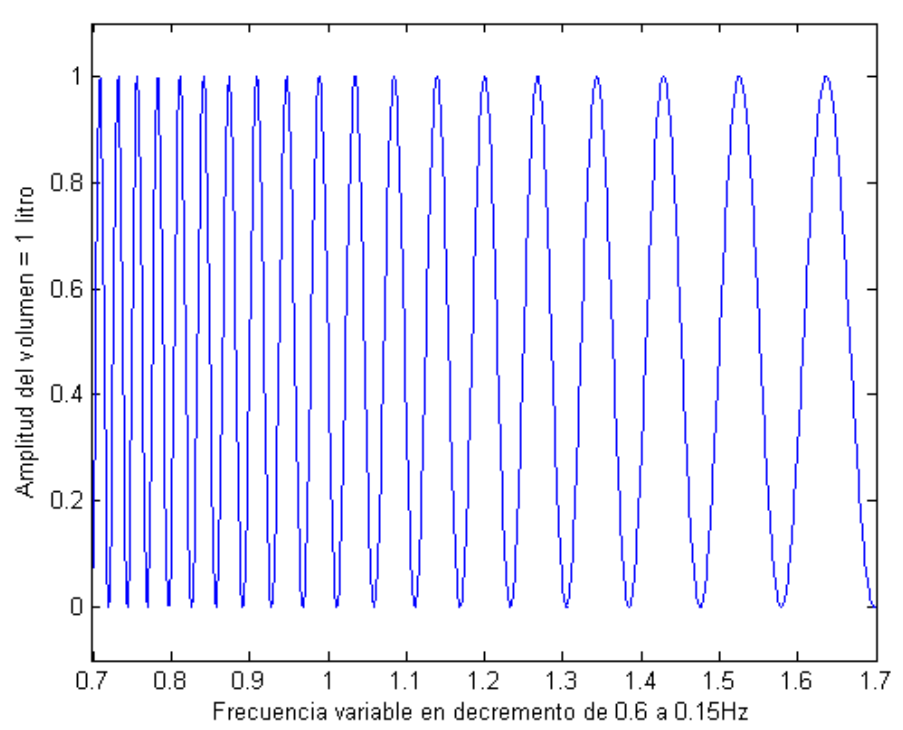

Figura 2.22. Patrón ACFVd.

Los parámetros a programar son:

1- Amplitud (A) en ml.

2- Frecuencia inicial y final (Fi, $\mathrm{Ff}$ ) en $\mathrm{Hz}$.

3- Tiempo de duración del patrón (T) en segundos.

\section{II.6 Validación del GPRV}

La validación del GPRV como técnica para evaluar la ASR, se llevó a cabo por medio de la realización de un diseño EXPERIMENTAL donde se midieron los errores absolutos en amplitud y frecuencia de respiración a respiración de tres patrones generados por MatLab ACFC, ACFVi y AViFC. La figura 2.23 muestra la forma de medición de los errores absolutos en amplitud y frecuencia de las respiraciones de los sujetos con respecto a los máximos en amplitud del patrón Xn y en frecuencia a partir de los periodos del patrón Xn generado en MatLab. 


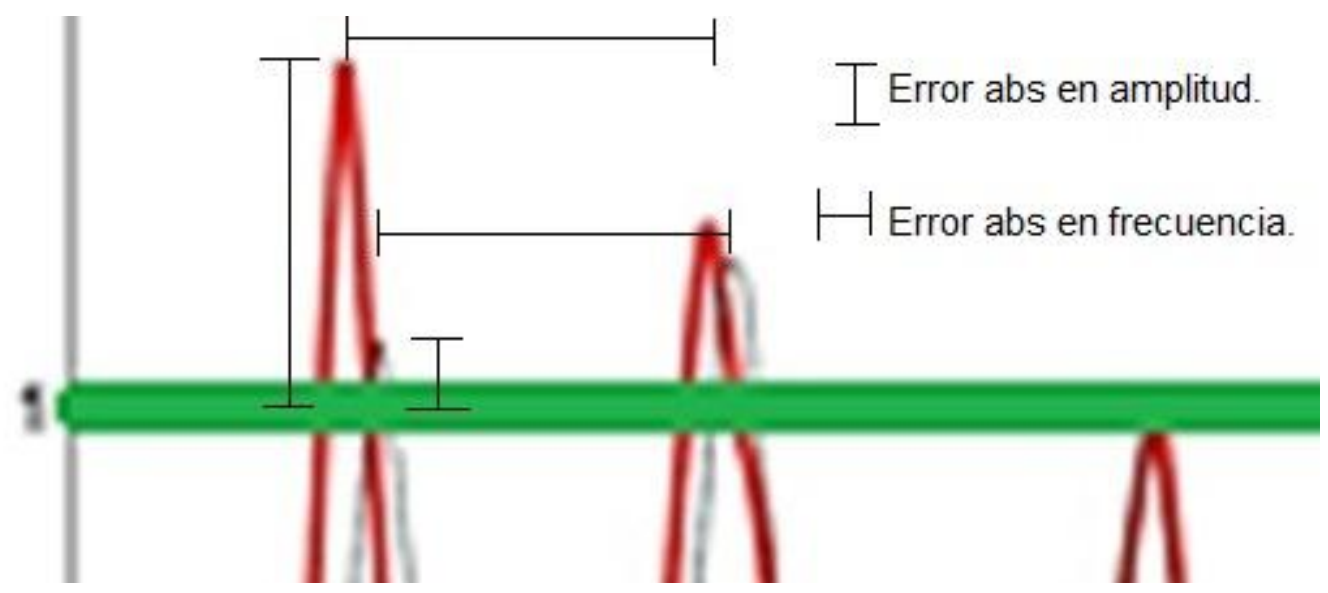

Figura 2.23. Errores absolutos respiración a respiración en amplitud del volumen y frecuencia respiratoria para el patrón ACFC, amplitud de 1 litro, frecuencia de $0.2 \mathrm{~Hz}$. En rojo se muestran las respiraciones de los sujetos bajo experimentación utilizando las columnas de LED del GPRV y en gris las respiraciones de los mismos sujetos con seguimiento visual en la pantalla de TFT.

Debido a que las dos generaciones de dispositivos anteriores al GPRV sólo son marcadores de frecuencia, nunca fueron medidos ni calibrados y su error absoluto promedio en frecuencia fue de \pm 0.19 con respecto al patrón Xn, mientras que el GPRV presentó ya calibrado un error absoluto promedio en frecuencia de \pm 0.001 con respecto a Xn; se decidió comparar los errores y desviaciones estándar solamente entre el GPRV y el método diseñado por Guillen Alejandra que consiste en la generación de patrones en MatLab mostrados en una pantalla TFT de 32" y su correspondiente seguimiento visual por los sujetos bajo experimentación.

\section{II.6.1 Diseño experimental}

\section{II.6.1.1 Sujetos de experimentación}

A todos los sujetos de la muestra aleatoria se les informó por escrito en qué consistía el protocolo, así como sus posibles riesgos, para que dieran su consentimiento informado conforme a la Declaración de Helsinki de la Asociación Médica Mundial. 
Se estudiaron 11 sujetos sanos, de género indistinto, con una muestra por "cuota y conveniencia", es decir de la muestra aleatoria se eligieron a los primeros sujetos que cumplieron con los requisitos de inclusión, que se mencionan en los siguientes párrafos.

Los requisitos de inclusión fueron:

1- Clínicamente sanos por historia clínica.

2- $\quad$ Sedentarios y no fumadores.

3- $\quad$ Sujetos entre 18 y 26 años.

4- $\quad$ Medidas antropométricas: talla de 1.60 a $1.75 \mathrm{~m}$, peso entre 57 y 74 .

5- $\quad$ Estado de salud confirmado por ECG de reposo y espirometría.

6- $\quad$ Normotensos y sin enfermedades respiratorias.

Los requisitos de exclusión fueron:

1- $\quad$ Sujetos menores a 18 y mayores a 26 años.

2- $\quad$ Con padecimientos crónicos.

3- Bajo régimen de medicamentos por algún padecimiento temporal.

4- $\quad$ Con peso superior a 74 o inferior a $57 \mathrm{~kg}$.

Los requisitos de rechazo fueron:

1- Bajo tratamiento de alguna enfermedad.

2- Con enfermedades respiratorias o cardiovasculares.

3- Con ingesta previa de medicamentos.

4- Mujeres con periodo menstrual.

\section{II.6.1.2 Protocolo de esfuerzo respiratorio}

1- Se les solicitó previo a la prueba:

a. No haber tomado café.

b. Desayunar ligero (1h antes del estudio, como mínimo).

c. No haber realizado ejercicio intenso. 
d. No haber tomado fármacos.

e. Que hayan dormido al menos $8 \mathrm{~h}$ la noche anterior.

2- Se usaron los siguientes patrones de respiración:

a. Patrón de respiración ACFC, amplitud de 1 litro, frecuencia de $0.2 \mathrm{~Hz}$ y tiempo de duración de 1 minuto. Figura 2.24.

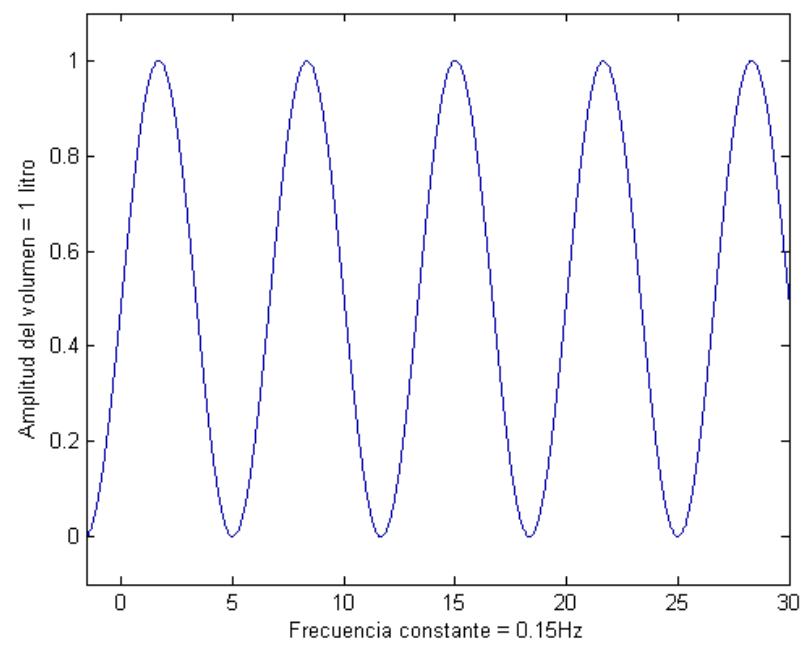

Figura 2.24. Patrón ACFC (1 litro, 0.15 Hz).

b. Patrón de respiración de ACFVi, amplitud de 1 litro, frecuencia variable de 0.15 a $0.6 \mathrm{~Hz}$ y tiempo de duración de 1 minuto. Figura 2.25 .

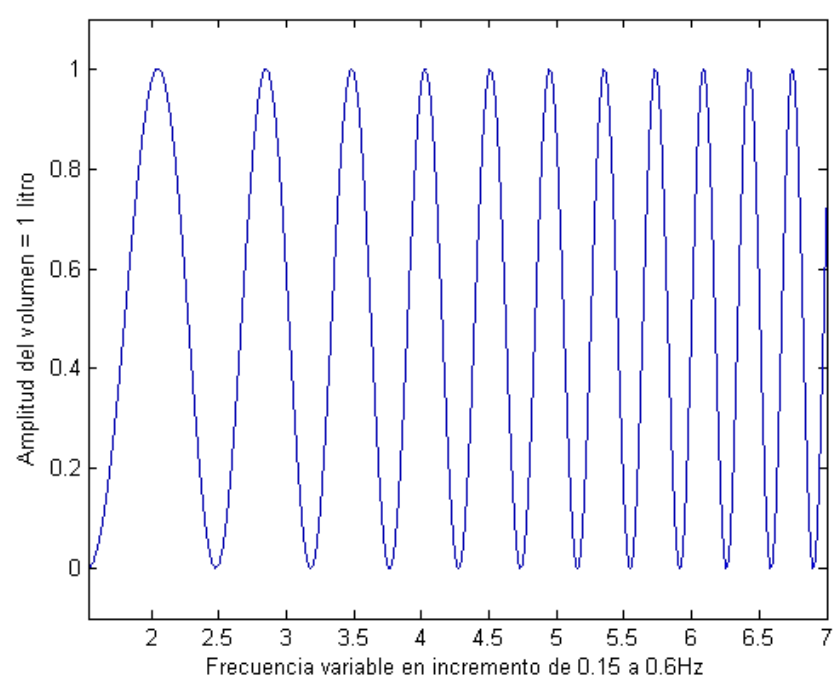

Figura 2.25. Patrón ACFVi (1 litro, 0.15 a 0.6 Hz). 
c. Patrón de respiración $\mathrm{AViFC}$, frecuencia de $0.2 \mathrm{~Hz}$ y amplitud variable de 0.2 a 2 litros. Figura 2.26 y tiempo de duración de 1 minuto.

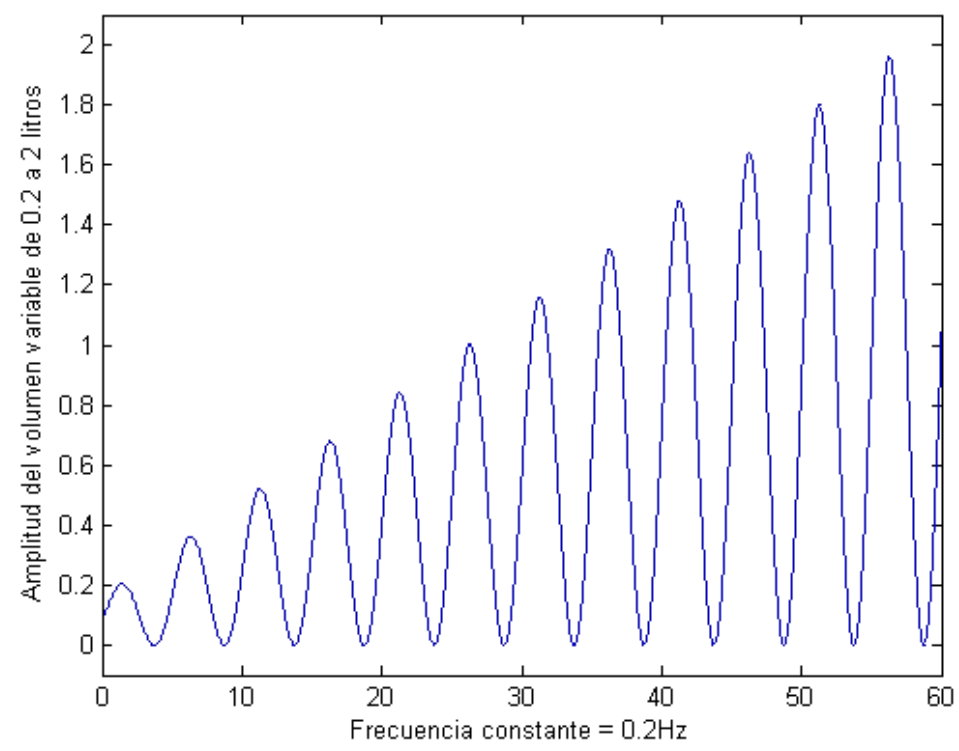

Figura 2.26. Patrón AViFC (0.2 a 2 litros, 0.2 Hz).

Entre cada maniobra de esfuerzo respiratorio se dejaron dos minutos con la finalidad de recuperar la función del sujeto.

Se utilizaron estos patrones de respiración debido a que como se mencionó en los antecedentes presentan un máximo en la densidad espectral de potencia y una mayor estimulación de la rama parasimpática además de ser ampliamente utilizados para poder caracterizar la ASR [5, 23, 24]. 


\section{II.7 Registro y adquisición}

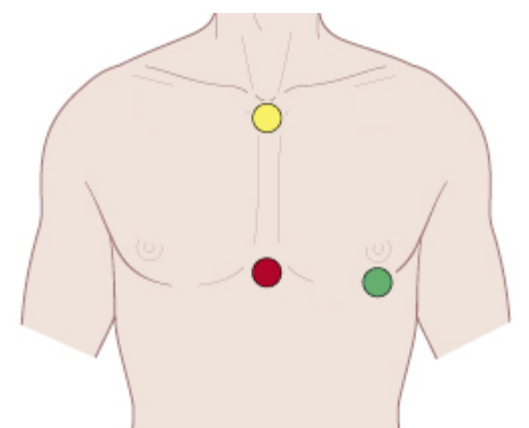

Figura 2.27. Derivación bipolar
El ECG se obtuvo por medio de la derivación bipolar torácica CM5. Se coloca el electrodo positivo (verde) en el sitio de la derivación V5, el electrodo negativo (amarillo) en el manubrio esternal y el electrodo de referencia (rojo) en el apéndice xifoides, como se muestra en la figura 2.27 . CM5.

Intencionalmente se usa esta derivación porque minimiza los artefactos causados por el movimiento y además maximiza las ondas R.

El acondicionamiento se realizó por medio de un amplificador BIOPAC modelo ECG100C, como el mostrado en la figura 2.28.

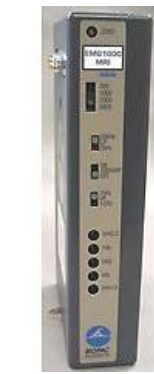

Figura BIOPAC ECG100.

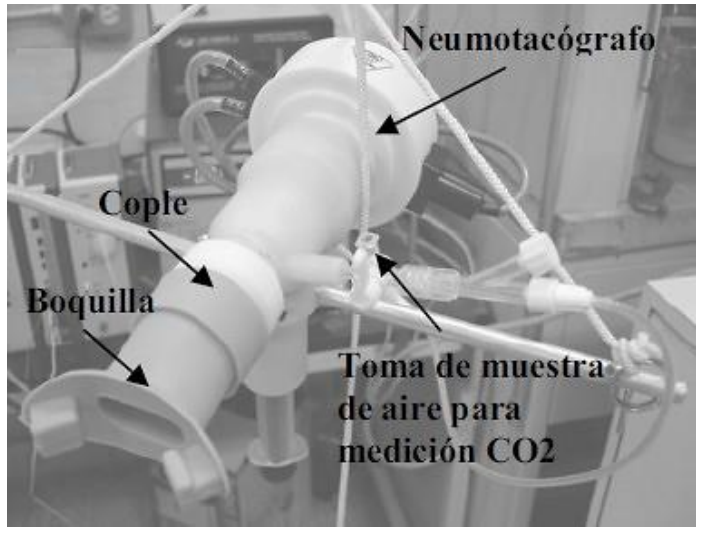

Figura 2.29. Neumotacómetro.
El volumen corriente se registró con el empleo de un neumotacómetro 3813 Hans Rudolph (figura 2.29), un transductor de presión diferencial MP45 Validyne y un demodulador de acarreo CD19A Validyne. 
La señal analógica se introdujo en un módulo BIOPAC $^{\circledR}$ MP100 vía el módulo HTL100C. Se utilizaron las herramientas del programa Acqknowledge para mostrar las señales. Figura 2.30 .

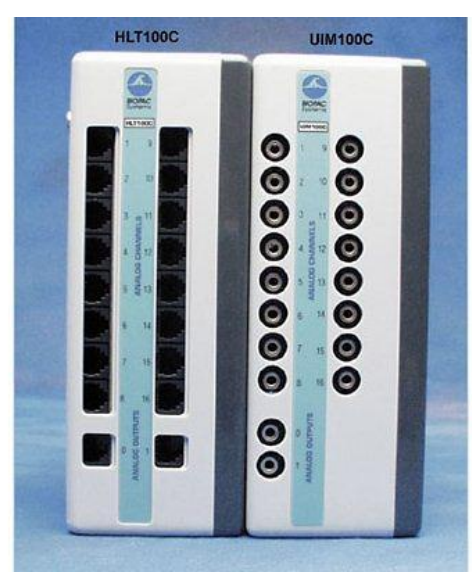

Figura 2.30. Módulos MP100 y HTL100C.

Todas las señales se muestrearon a $500 \mathrm{~Hz}$ y se almacenaron para su procesamiento fuera de línea.

\section{II.8 Procesamiento de señales}

El tacograma es una opción que provee el procesamiento del software Acqnowledge (BIOPAC ${ }^{\circledR}$ ), a partir de éste se realiza el siguiente procedimiento:

1) Eliminación de la tendencia.

2) A partir de la anterior se construyeron las series de tiempo cuyo rango de variación es la ASR. 


\section{CAPÍTULO III RESULTADOS Y DISCUSIÓN}

"El asunto es el problema; la forma, la solución". 


\section{CAPÍTULO III}

\section{RESULTADOS}

\section{III.1 Costos del sistema embebido}

Tabla 3.1 relación beneficio-costo del sistema embebido lightuino.

\begin{tabular}{|l|r|}
\hline Beneficios & $\begin{array}{r}\text { Cuantificación } \\
\text { \$MN }\end{array}$ \\
\hline $\begin{array}{l}\text { Sistema embebido con base en microcontrolador y } \\
\text { controlador de LED. }\end{array}$ & 3600 \\
\hline Software compilador IDE sin costo de licencia. & 5006 \\
\hline Compatible con software arduino. & 1000 \\
\hline Compatible con shields de arduino. & 1000 \\
\hline Totales & 10606 \\
\hline Contra-beneficios & 1800 \\
\hline Costo del sistema embebido & 6000 \\
\hline Costos de programación & 7800 \\
\hline Totales & 1.4 \\
\hline Relación beneficio-costo B/C = Beneficios/Costos & \\
\hline
\end{tabular}

La tabla 3.1 muestra los beneficios y contra-beneficios o costos más importantes del sistema seleccionado. Se obtiene una relación beneficio-costo simple de 1.4 lo que indica que la selección fue buena. Una relación $B / C>1$ indica que un proyecto es aceptado financieramente. 
Tabla 3.2 Comparación de costos

del sistema embebido lightuino

y el kit PIC $® 16 F 877 A$

\begin{tabular}{|l|c|}
\hline Sistema & Costo $\$$ \\
\hline PIC $^{\circledR} 16$ F877A KIT & $\$ 7,037$ \\
\hline Lightuino $^{\circledR}$ & $\$ 1,800$ \\
\hline Diferencia en costo & $\mathbf{\$ 5 , 2 3 7}$ \\
\hline
\end{tabular}

La tabla 3.2 muestra una diferencia del 75\% más en costo para un kit de desarrollo con PIC respecto del sistema embebido lightuino.

\section{III.2 Cifras de mérito del GPRV}

Se logró tener la posibilidad de programar múltiples secuencias de patrones respiratorios con frecuencias fijas y variables (barridos en incremento y decremento lineal) y amplitudes constantes y variables (barridos en incremento y decremento lineal) con un error absoluto promedio en frecuencia de 0.003 y 0.001 en amplitud.

Tabla 3.3. Caracterización del GPRV.

\begin{tabular}{|c|c|c|}
\hline \multirow{2}{*}{$\begin{array}{l}\text { Condiciones de } \\
\text { temperatura ambiente } \\
\text { Alimentación 9V }\end{array}$} & $\mathrm{F}=0.15 \mathrm{a} 0.40$ & $\begin{array}{c}\mathrm{A}=500 \text { a } 3500 \mathrm{ml} \\
\text { Resolución de } 50 \mathrm{ml} / \mathrm{LED}\end{array}$ \\
\hline & $\begin{array}{c}\text { Frecuencia } \\
\mathrm{Hz}\end{array}$ & $\begin{array}{l}\text { Amplitud } \\
\text { Litros }\end{array}$ \\
\hline Rango 9 a 24 rpm & 0.15 a 0.40 & 0.5 a 2 \\
\hline $\begin{array}{l}\text { Alcance o Span } \\
24-9 \text { rpm = } 15\end{array}$ & $0.40-0.15=\mathbf{0 . 2 5}$ & $2-0.5=1.5$ \\
\hline $\begin{array}{l}\text { Error absoluto } \\
\text { promedio } \\
\text { (medición con } \\
\text { osciloscopio) } \\
\text { error abs = |Yno }-\mathrm{Xn} \mid \\
\end{array}$ & $\begin{array}{c} \pm 0.002 \text { con respecto al rango } \\
\text { dinámico }\end{array}$ & $\begin{array}{c} \pm 0.003 \text { con respecto al rango } \\
\text { dinámico } \\
x[\text { litros }]=\frac{2 l(1.558 \mathrm{~V})}{1.56 \mathrm{~V}}=1.997 l \\
2 l-1.997 l=0.003 l\end{array}$ \\
\hline $\begin{array}{c}\begin{array}{c}\text { Desviación estándar } \\
\text { (medición con } \\
\text { osciloscopio) }\end{array} \\
d s=\sqrt{\frac{\sum\left(Y_{n o}-\overline{Y_{n o}}\right)^{2}}{(n-1)}}\end{array}$ & $\begin{array}{c} \pm 0.080 \text { con respecto al rango } \\
\text { dinámico }\end{array}$ & \pm 0.001 \\
\hline $\begin{array}{c}\text { Precisión } \\
e P_{n k}=Y_{n k}-Y_{n}\end{array}$ & $\pm 0.2 \%$ & $\pm 0.4 \%$ \\
\hline $\begin{array}{c}\text { Exactitud } \\
e E_{n k}=Y_{n k}-X_{n}\end{array}$ & $\pm 0.3 \%$ & $\pm 0.7 \%$ \\
\hline
\end{tabular}


La tabla 3.3 muestra el rango, alcance o span, error absoluto promedio y errores absolutos promedio de precisión y exactitud

Las cifras de mérito del GPRV ya calibrado en amplitud y frecuencia se muestran en la tabla 3.4, 3.5 Y 3.6.

Tabla 3.4. Cifras de mérito del GPRV, patrón ACFC.

\begin{tabular}{|c|c|c|}
\hline \multicolumn{3}{|c|}{$\begin{array}{c}\text { Condiciones de temperatura ambiente. } \\
\text { Alimentación 9V } \\
\text { Protocolo: ACFC } \\
\text { A = 3500 ml }\end{array}$} \\
$\mathrm{F}=0.15$ a $0.40 \mathrm{~Hz}$ (Rango) \\
\hline $\begin{array}{c}\text { Cifras de mérito } \\
\text { Precisión promedio } \\
1-\sigma_{\varepsilon_{P}}\end{array}$ & $\begin{array}{c}0.992 \\
\text { con respecto al Rango }\end{array}$ & 0.991 \\
\hline $\begin{array}{c}\text { Exactitud promedio } \\
1-\sigma_{\varepsilon_{E}}\end{array}$ & $\begin{array}{c}0.985 \\
\text { con respecto al Rango }\end{array}$ & 0.986 \\
\hline $\begin{array}{c}\text { Certeza } \\
1-\left|\varepsilon_{C}\right|\end{array}$ & 0.998 & 0.996 \\
\hline
\end{tabular}

Tabla 3.5. Cifras de mérito del GPRV, patrón ACFVi.

\begin{tabular}{|c|c|c|}
\hline \multicolumn{3}{|c|}{$\begin{array}{c}\text { Condiciones de temperatura ambiente. } \\
\text { Alimentación 9V } \\
\text { Protocolo: ACFVi } \\
\text { A = } 1000 \mathrm{ml}\end{array}$} \\
$\mathrm{F}=0.15 \mathrm{a} 0.40 \mathrm{~Hz}$ (Rango) \\
\hline Cifras de mérito & Frecuencia & Amplitud \\
\hline $\begin{array}{c}\text { Precisión promedio } \\
1-\sigma_{\varepsilon_{P}}\end{array}$ & $\begin{array}{c}0.996 \\
\text { con respecto al Rango }\end{array}$ & 0.994 \\
\hline $\begin{array}{c}\text { Exactitud promedio } \\
1-\sigma_{\varepsilon_{E}}\end{array}$ & $\begin{array}{c}0.988 \\
\text { con respecto al Rango }\end{array}$ & 0.987 \\
\hline $\begin{array}{c}\text { Certeza } \\
1-\left|\varepsilon_{C}\right|\end{array}$ & 0.998 & 0.997 \\
\hline
\end{tabular}

Tabla 3.6. Cifras de mérito del GPRV, patrón AViFC.

\begin{tabular}{|c|c|c|}
\hline \multicolumn{3}{|c|}{ Condiciones de temperatura ambiente. } \\
Alimentación 9V \\
Protocolo: AViFC \\
$\mathrm{A}=200 \mathrm{a} 1000 \mathrm{ml}$ \\
$\mathrm{F}=0.15$ a $0.40 \mathrm{~Hz}$ (Rango) \\
\hline Cifras de mérito & Frecuencia & Amplitud \\
\hline $\begin{array}{c}\text { Precisión promedio } \\
1-\sigma_{\varepsilon_{P}}\end{array}$ & $\begin{array}{c}0.995 \\
\text { con respecto al Rango }\end{array}$ & 0.993 \\
\hline $\begin{array}{c}\text { Exactitud promedio } \\
1-\sigma_{\varepsilon_{E}}\end{array}$ & $\begin{array}{c}0.987 \\
\text { con respecto al Rango }\end{array}$ & 0.987 \\
\hline $\begin{array}{c}\text { Certeza } \\
1-\left|\varepsilon_{C}\right|\end{array}$ & 0.997 & 0.998 \\
\hline \multicolumn{3}{|c|}{$\mid$} \\
\hline
\end{tabular}




\section{III.3 Energía consumida}

La potencia consumida aproximada del GPRV fue de 17.46W.

Si la comparamos con el método de TFT que tiene un consumo aproximado de $120 \mathrm{~W}$ por computadora podemos decir que el GPRV presenta un menor consumo de energía.

\section{III.4 Portabilidad}

La portabilidad es la facilidad con que se puede transportar un equipo, entonces el GPRV con dimensiones de $33 \mathrm{~cm}$ de alto por $5 \mathrm{~cm}$ de ancho y con un peso total considerando las baterías de $1.2 \mathrm{~kg}$, presenta una alta portabilidad con respecto a los dos anteriores marcadores de tiempo y por supuesto a las dos computadoras personales y la pantalla de TFT de 32 ".

\section{III.5 Comparación de técnicas de caracterización por GPRV y computadora}

La figura 3.1 muestra la superposición del patrón de respiración ACFC de uno de los sujetos por el método de referencia (gris) y por el método del GPRV (rojo). 


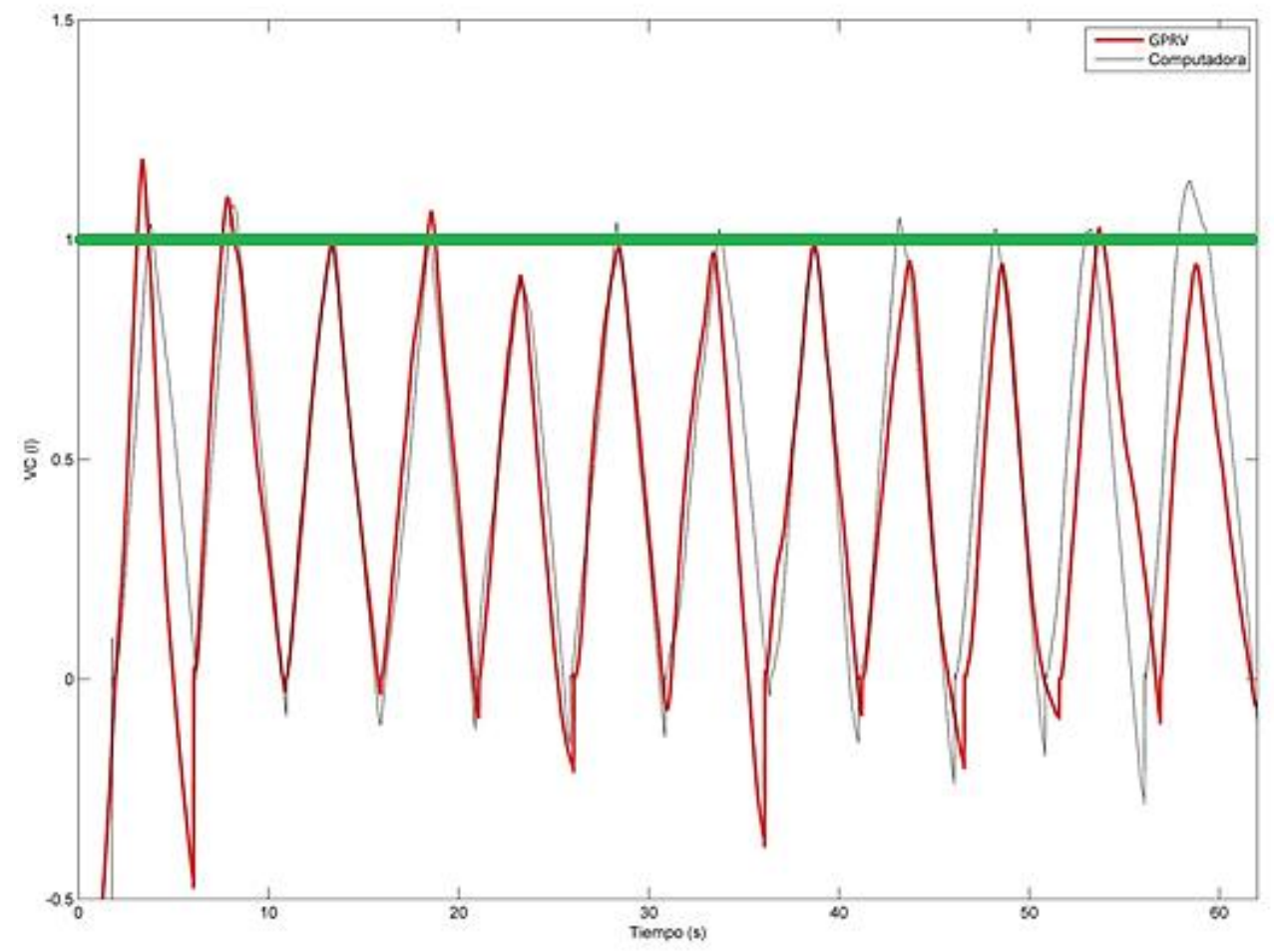

Figura 3.1. Superposición del patrón ACFC. Método de computadora y GPRV.

El seguimiento del sujeto en frecuencia y amplitud es similar al método por computadora (visualización y seguimiento en pantalla TFT) y se confirma en los resultados de la tabla 3.7 .

\begin{tabular}{l|l|l}
\hline $\begin{array}{c}\text { Tabla 3.7. Valores medios de los errores } \\
\text { absolutos para el protocolo ACFC. }\end{array}$ \\
\hline \multicolumn{2}{c}{ ACFC } \\
\hline & errF & errA \\
\hline Computadora & 0.03 & 0.034 \\
\hline ds & 0.03 & 0.03 \\
\hline GPRV & 0.02 & 0.042 \\
\hline ds & 0.02 & 0.046 \\
\hline & & \\
\hline Dif (respecto a ds) & $1 \%$ & $1.6 \%$ \\
\hline
\end{tabular}

La tabla 3.7 muestra los valores promedio de los errores absolutos para el protocolo ACFC, en frecuencia (errF) y en amplitud del volumen (errA), sus desviaciones estándar (ds), así como la diferencia en porcentaje respecto de la ds. La figura 3.2 muestra la superposición del patrón de respiración ACFVi de uno de los sujetos por el método de computadora (gris) y por el método del GPRV (rojo). 


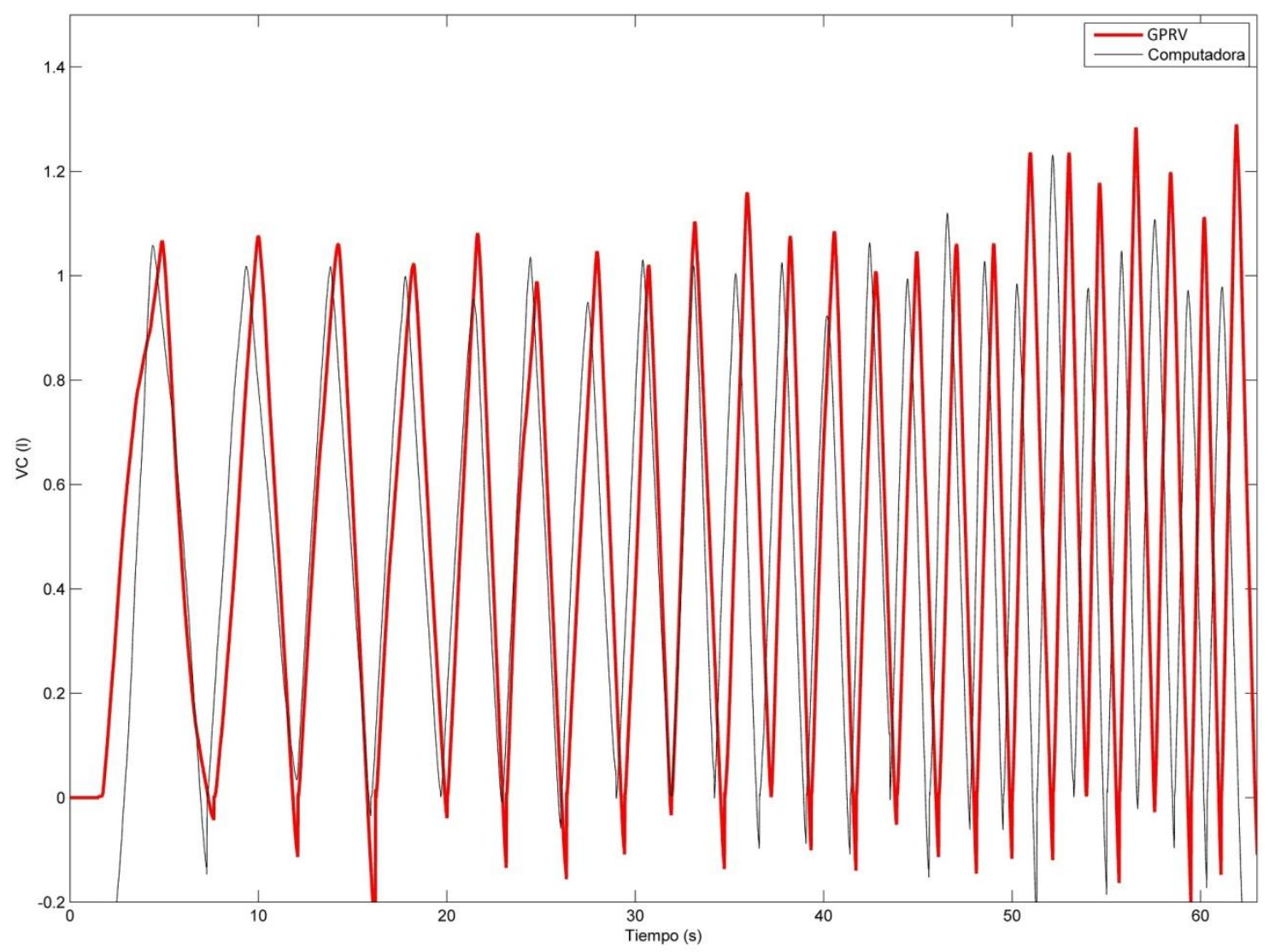

Figura 3.2. Superposición del patrón ACFVi. Método de computadora y GPRV.

Nuevamente el seguimiento del sujeto es muy similar al método de referencia confirmado en la tabla 3.8.

\begin{tabular}{|c|c|c|}
\hline \multicolumn{3}{|c|}{$\begin{array}{l}\text { Tabla 3.8. Valores medios de los errores } \\
\text { absolutos para el protocolo ACFVi. }\end{array}$} \\
\hline & \multicolumn{2}{|c|}{ ACFVi } \\
\hline & errF & errA \\
\hline Computadora & 0.082 & 0.057 \\
\hline $\mathrm{ds}$ & 0.059 & 0.045 \\
\hline GPRV & 0.076 & 0.077 \\
\hline ds & 0.052 & 0.062 \\
\hline Dif (respecto a ds) & $0.7 \%$ & $1.7 \%$ \\
\hline
\end{tabular}

La tabla 3.8 muestra los valores promedio de los errores absolutos para el protocolo ACFVi, en frecuencia (errF) y en amplitud del volumen (errA), sus desviaciones estándar (ds), así como la diferencia en porcentaje respecto de la ds.

Finalmente la figura 3.3 muestra la superposición del patrón de respiración AViFC de uno de los sujetos por el método de computadora (gris) y por el método del GPRV (rojo). 


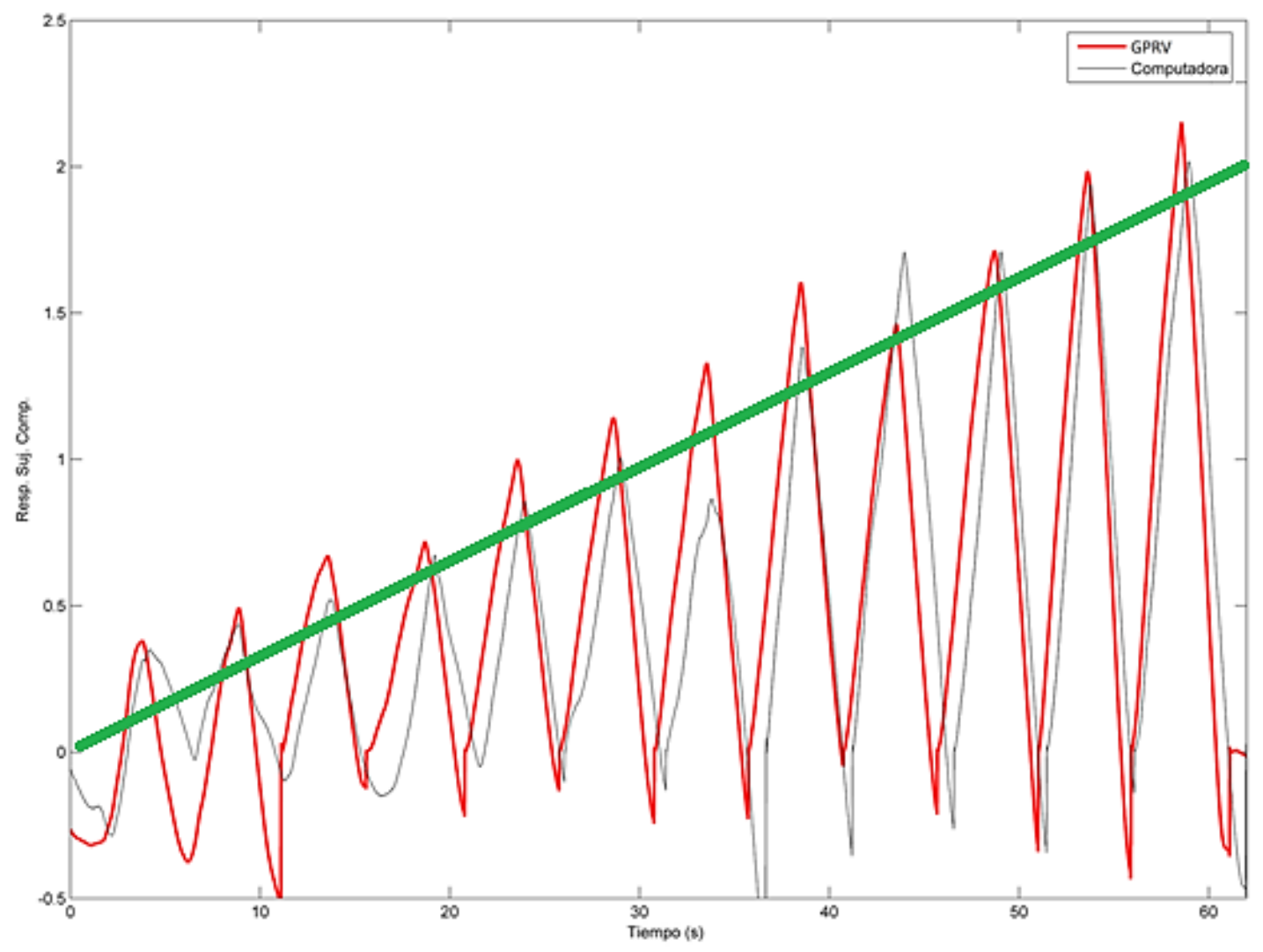

Figura 3.3. Superposición del patrón AViFC. Método de computadora y GPRV.

El seguimiento del sujeto muestra también que es muy parecido al método de referencia y se confirma cuantitativamente en la tabla 3.9.

\begin{tabular}{l|l|l}
\hline $\begin{array}{c}\text { Tabla 3.9. Valores medios de los errores } \\
\text { absolutos para el protocolo AViFC. }\end{array}$ \\
\hline \multicolumn{2}{c}{ AViFC } \\
\hline & errF & errA \\
\hline Computadora & 0.03 & 0.057 \\
\hline ds & 0.02 & 0.062 \\
\hline GPRV & 0.02 & 0.084 \\
\hline ds & 0.02 & 0.075 \\
\hline & & \\
\hline Dif (respecto a ds) & $0 \%$ & $1.3 \%$ \\
\hline
\end{tabular}

La tabla 3.9 muestra los valores medios de los errores absolutos para el protocolo AViFC, en frecuencia (errF) y en amplitud del volumen (errA), sus desviaciones estándar (ds), así como la diferencia en porcentaje respecto de la ds.

En la tabla 3.8 se listan los valores promedio del error (error en frecuencia y amplitud: errF, errA) con sus correspondientes desviaciones estándar (ds) para ambos procedimientos (método de computadora con visualización en pantalla TFT 
y método con GPRV). Se cuantifican las diferencias (dif) con respecto a ds de ambos procedimientos como porcentaje en la última fila de la tabla 3.10.

Tabla 3.10. Valores medios de los errores absolutos para los tres protocolos.

\begin{tabular}{|c|c|c|c|c|c|c|}
\hline & \multicolumn{2}{|c|}{ ACFC } & \multicolumn{2}{|c|}{ ACFVi } & \multicolumn{2}{|c|}{ AViFC } \\
\hline & errF & errA & errF & errA & errF & errA \\
\hline Computadora & 0.03 & 0.034 & 0.082 & 0.057 & 0.03 & 0.057 \\
\hline ds & 0.03 & 0.03 & 0.059 & 0.045 & 0.02 & 0.062 \\
\hline GPRV & 0.02 & 0.042 & 0.076 & 0.077 & 0.02 & 0.084 \\
\hline ds & 0.02 & 0.046 & 0.052 & 0.062 & 0.02 & 0.075 \\
\hline Dif (respecto a ds) & $1 \%$ & $1.6 \%$ & $0.7 \%$ & $1.7 \%$ & $0 \%$ & $1.3 \%$ \\
\hline
\end{tabular}

La comparación de ambos métodos reveló:

1- El valor promedio del error de ambos procedimientos fue similar en las tres maniobras.

2- El error fue pequeño y similar entre los dos procedimientos.

Las figuras 3.4, 3.5 y 3.6 muestran la dinámica de la ASR de los tres protocolos de respiración realizados por medio del GPRV.

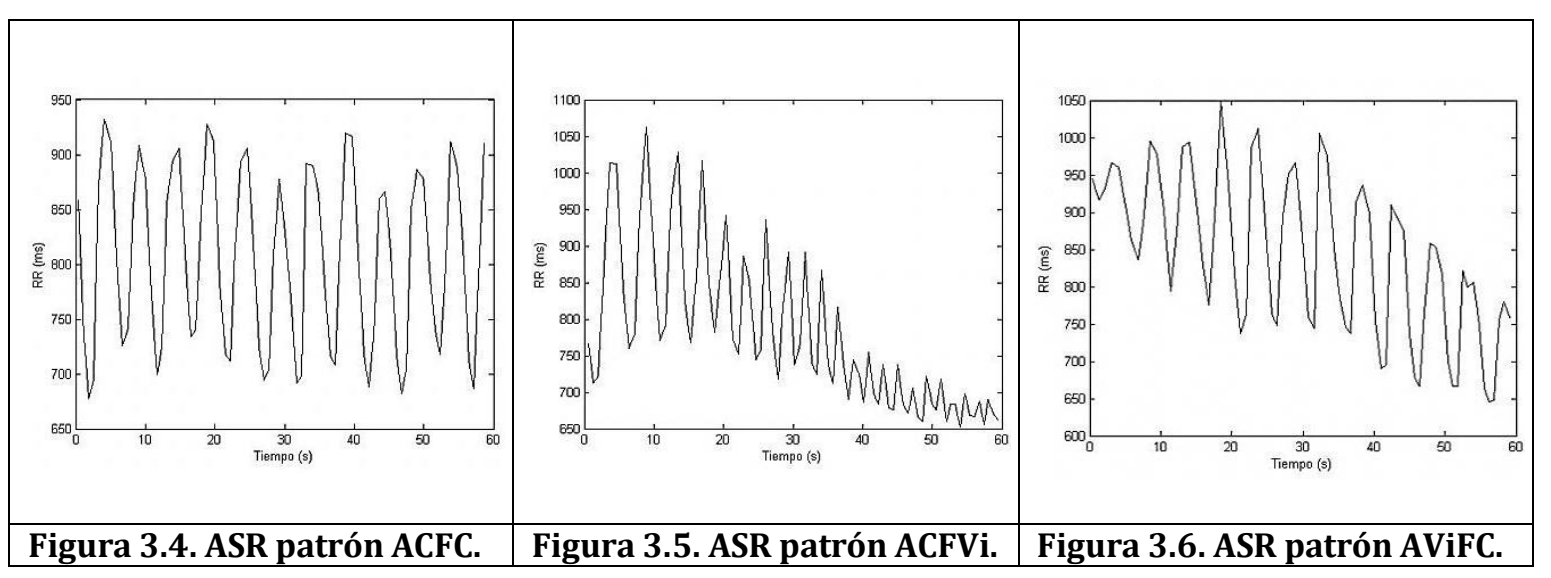


El análisis demostró:

1- Los valores medios de la ASR fueron muy similares entre las tres maniobras, hecho que se muestra en la tabla 3.11.

Tabla 3.11. Valores medios de la ASR.

\begin{tabular}{cccc}
\hline & $\begin{array}{c}\text { FR constante, VC } \\
\text { constante }\end{array}$ & $\begin{array}{c}\text { FR creciente, VC } \\
\text { constante }\end{array}$ & $\begin{array}{c}\text { FR constante, VC } \\
\text { creciente }\end{array}$ \\
\hline Computadora & $146 \pm 42$ & $86 \pm 33$ & $141 \pm 45$ \\
GPRV & $145 \pm 63$ & $87 \pm 28$ & $132 \pm 56$
\end{tabular}

2- Con relación a la maniobra ACFVi (figura 3.2) la ASR disminuyó con el incremento de la frecuencia. Resultado acorde con el procedimiento de referencia publicado por Guillen Mandujano y Carrasco Sosa en "Interactive Effects of Simultaneously Varying Respiratory Frequency and Tidal Volume on Respiratory Sinus Arrhythmia". Computing in Cardiology; 39:165-168, 2012 [5].

3- En la maniobra AViFC la ASR resultó ser muy parecida en ambos procedimientos. El efecto en los cambios en el VC sobre la ASR es controversial. Comúnmente se acepta que la magnitud de la ASR se incrementa proporcionalmente a los incrementos en VC (Grossman y Taylor 2007). Sin embargo, otros autores (Guillén-Mandujano y Carrasco-Sosa 2014) han documentado que existe un límite de VC más allá del cual la amplitud de la ASR decae, y que los cambios debidos a modificaciones voluntarias crecientes y decrecientes del VC son ambiguos y presentan histéresis.

La linealidad de esta relación se soporta en el hecho de que la amplitud de la ASR es normalizada por el VC; un procedimiento extensivamente realizado utilizando varias técnicas [25]. 


\section{DISCUSIÓN}

\section{III.6 Principales Hallazgos}

1- La relación beneficio costo de 1.4 demuestra que el sistema embebido empleado a pesar de sus limitaciones en hardware y software con respecto de las computadoras es financieramente aceptable.

2- Las cifras de mérito después de la calibración indican que la calibración en frecuencia y amplitud cumplieron con el objetivo de tener mejoras considerables respecto de los dos marcadores de tiempo anteriores.

3- La energía consumida por el GPRV permite alimentar con pilas recargables lo que aumenta su portabilidad.

4- La programación del GPRV logró reproducir adecuadamente patrones respiratorios en amplitud y frecuencia constante y variable por medio de una columna vertical visual de LED. Dichos patrones son necesarios para la estimulación de la actividad vagal cardiaca.

5- Las funciones programadas en el GPRV ajusta adecuadamente los valores al patrón de referencia sin la necesidad de hardware excesivo como con el método de computadora y pantalla TFT. Y sin el uso de licencias de software costosas.

6- No es necesaria una etapa extra de procesamiento y generación de un patrón analógico.

7- Los patrones de respiración aplicados en la experimentación mostraron ser bastante eficientes a pesar del hardware y software limitado y debido a que los sujetos no fueron entrenados previamente.

8- Las tres maniobras experimentales no presentaron diferencias significativas en cuanto al valor promedio del error con respecto al método de computadora.

9- La relación entre el VC y la ASR manifestaron un comportamiento lineal característico. 


\section{III.7 Aportes al conocimiento fisiológico}

1. El GPRV como estimulador de la modulación vagal mostró ser adecuado al incrementar o decrementar la función de la rama vagal cardiaca.

2. La estimulación adecuada podría arrojar patrones normales en sujetos sanos que en la clínica serían de gran utilidad al diagnóstico oportuno de algunos padecimientos cardiacos. 


\section{CAPÍTULO IV CONCLUSIONES}

"Es una cosa bastante repugnante el éxito. Su falsa semejanza con el mérito engaña a los hombres"

- Victor Hugo | Francia 


\section{CAPÍTULO IV}

\section{CONCLUSIONES}

1. Las evidencias encontradas en la presente tesis son contundentes y satisfactorias al hecho de que el GPRV coadyuvó en la simplificación en hardware, software, costos y portabilidad en la estimulación de la rama vagal cardiaca.

2. Esta simplificación permitió observar linealidad en la relación de la ASR y el incremento en el VC.

3. El desempeño del GPRV brindó mejores resultados que en los dos dispositivos anteriores y similares a los obtenidos por medio del método de computadora.

\section{INVESTIGACIONES POSTERIORES}

La automatización en el proceso de coordinación entre el método de computadora y el GPRV puede reducir considerablemente el error entre el patrón y el seguimiento respiratorio del sujeto.

Para que el GPRV sea completamente portable es indispensable diseñar un neumotacómetro inalámbrico y utilizar otro sistema embebido con mayor poder de procesamiento y con sistema operativo embebido. 


\section{OTRAS POSIBLES ÁREAS DE BENEFICIO}

1- Puede ser posible usar la programación del GPRV para minimizar los efectos negativos que producen las máquinas de respiración artificial y con ello coadyuvar en el mejoramiento de la calidad de vida de los pacientes sujetos a este procedimiento que sufren de lesiones en la pleura debido a una respiración sin variaciones en frecuencia y amplitud.

2- Es factible usar en sesiones cortas de tiempo los patrones de respiración para mejorar el intercambio de gases pulmonares a nivel alveolar.

3- Un uso en la recuperación de pacientes con pérdidas de coordinación motriz debido a fallos en un mecanismo neuronal en el cerebelo puede ser factible. 


\section{Lista de tablas y figuras}

\section{Capítulo I}

\section{$\underline{\text { Figuras }}$}

Figura 1.1. Caracterización de la ASR mininvasiva.

Figura 1.2. Sistema embebido Lightuino.

Extraída de:

http://www.toastedcircuits.com/html/product/Lightuino_3.0.html

Figura 1.3. Importancia del control de la respiración.

Extraída de:

http://amf-semfyc.com/web/article_ver.php?id=1077

Figura 1.4. Intercambio de gases pulmonares a nivel alveolar.

Extraída de:

http://recursostic.educacion.es/ciencias/biosfera/web/alumno/3ESO/diges/contenidos10.htm

Figura 1.5. Montaje experimental de Hirsch y Bishop [2].

Figura 1.6. Influencia de la frecuencia respiratoria sobre la ASR [3].

Figura 1.7. Dispositivo electrónico con barra de LED.

Figura 1.8. Patrones respiratorios visuales experimentales superpuestos sobre los generados [5].

Figura 1.9. Patrones similares del periodo de la respiración y la ASR de diferentes tareas de esfuerzo respiratorio [6].

Figura 1.10. Modelo simulado de la relación entre la ASR y las descargas del nervio vago [5].

Figura 1.11. Influencia de los NGPR sobre la ASR.

Figura 1.12. Construcción de la serie de tiempo VFC.

Figura 1.13. Diagnosis de padecimientos cardiovasculares.

Extraída de:

Origen desconocido.

Figura 1.14. Comportamiento espectral de la VFC.

Figura 1.15. Hardware y software del método usado para el control de la respiración.

\section{Capítulo II}

$\underline{\text { Tablas }}$

Tabla 2.1. Comparativos de cuatro sistemas embebidos basados en microcontrolador en el año 2014.

Tabla 2.2. Diseño de la etapa de acondicionamiento del neumotacómetro.

Tabla 2.3. Frecuencias a programar en el GPRV.

Tabla 2.4. Rango dinámico del GPRV.

Tabla 2.5. Cálculo de los errores de los valores promedio de cada frecuencia. 


\section{$\underline{\text { Figuras }}$}

Figura 2.1. Comportamiento espectral de la VFC.

Figura 2.2. Diagrama general del GPRV.

Figura 2.3. Diseño PCB Módulo visual GPRV.

Figura 2.4. Efectos del cambio de postura en al análisis espectral de la

frecuencia cardiaca [37].

Figura 2.5. GPRV con un módulo lightuino.

Figura 2.6. GPRV montado en el Lab. de Fis. Médica.

Figura 2.7. Columnas de LED.

Figura 2.8. Campo visual del GPRV.

Figura 2.9. Amplificador de acondicionamiento del neumotacómetro.

Figura 2.10. Amplificador de acondicionamiento con etapa de ajuste.

Figura 2.11. Mapeo del volumen respiratorio a la columna de LED.

Figura 2.12. Comportamiento espectral de la ASR.

Figura 2.13a Conexión del osciloscopio al GPRV.

Figura 2.13b tiempos tomados con el osciloscopio del patrón del GPRV.

Figura 2.14 Patrón Xn lineal, tendencia y ajuste Yno (mediciones del osciloscopio) y tendencia y ajuste Yns (mediciones por monitor arduino) después de la calibración del GPRV.

Figura 2.15. Volúmenes y capacidades pulmonares

Extraída de:

Fisiología Médica, Guyton, Hall.

Figura 2.16. Amplitud del volumen constante.

Figura 2.17. Amplitud del volumen variable en incremento lineal.

Figura 2.18. Patrón ACFC.

Figura 2.19. Patrón AViFC.

Figura 2.20. Patrón AVdFC.

Figura 2.21. Patrón ACFVi.

Figura 2.22. Patrón ACFVd.

Figura 2.23. Errores absolutos respiración a respiración en amplitud del volumen y frecuencia respiratoria para el patrón ACFC, amplitud de 1 litro, frecuencia de $0.2 \mathrm{~Hz}$.

Figura 2.24. Patrón ACFC (1 litro, $0.15 \mathrm{~Hz})$.

Figura 2.25. Patrón ACFVi (1 litro, 0.15 a 0.6 Hz).

Figura 2.26. Patrón AViFC (0.2 a 2 litros, 0.2 Hz).

Figura 2.27. Derivación bipolar CM5.

Figura 2.28. BIOPAC ECG100.

Extraída de:

http://biopac-sys.jp/application/images/mri_emgamp.jpg

Figura 2.29. Neumotacómetro.

Figura 2.30. Módulos MP100 y HTL100C.

Extraída de:

http://www.lintoninst.co.uk/Portals/0/productimages/485_18262.jpg 


\section{Capítulo III}

Tablas

Tabla 3.1 relación beneficio-costo del sistema embebido lightuino.

Tabla 3.2 Comparación de costos del sistema embebido lightuino y el kit PIC $₫$ 16F877A.

Tabla 3.3. Caracterización del GPRV.

Tabla 3.4. Cifras de mérito del GPRV, patrón ACFC.

Tabla 3.5. Cifras de mérito del GPRV, patrón ACFVi.

Tabla 3.6. Cifras de mérito del GPRV, patrón AViFC.

Tabla 3.7. Valores medios de los errores absolutos para el protocolo ACFC.

Tabla 3.8. Valores medios de los errores absolutos para el protocolo ACFVi.

Tabla 3.9. Valores medios de los errores absolutos para el protocolo AViFC.

Tabla 3.10. Valores medios de los errores absolutos para los tres protocolos.

Tabla 3.11. Valores medios de la ASR.

\section{$\underline{\text { Figuras }}$}

Figura 3.1. Superposición del patrón ACFC. Método de computadora y GPRV.

Figura 3.2. Superposición del patrón ACFVi. Método de computadora y GPRV.

Figura 3.3. Superposición del patrón AViFC. Método de computadora y GPRV.

Figura 3.4. ASR patrón ACFC.

Figura 3.5. ASR patrón ACFVi.

Figura 3.6. ASR patrón AViFC. 


\section{Referencias Bibliográficas}

[1] Carrasco Sosa S., Gaitán González M. J., González Camarena R., Yañez Suárez O., Ríos Amezcua E., "Dispositivo electrónico para el control de la frecuencia respiratoria: Impacto en la reproducibilidad de la variabilidad de la frecuencia cardiaca". Revista Mexicana de Ingeniería Biomédica; Vol XXII, Núm. 2, pp 61-66, 2001.

[2] Castellanos Rodríguez A., Lobos Bejarano J. M., "Variantes de la normalidad". GdT Enfermedades cardiovasculares de la semFYC, AMF 2012;8(10):547-548, España 2012.

[3] Fumihiko Yasuma, MD, FCCP and Jun-ichiro Hayano, MD, FCCP, "Respiratory Sinus Arrhythmia- Why Does the Heartbeat Synchronize With Respiratory Rhythm". Chest 125:683-690, 2004.

[4] Cabrales Neira Mauricio F., Vanegas Cadavid Diego I., "Manual de métodos diagnósticos en electrofisiología cardiovascular". Sociedad Colombiana de Cardiología y Cirugía Cardiovascular, 15-20, 93-97, 2006.

[5] Angelone A, Coulter N. "Respiratory sinus arrhythmia: A frequency dependent phenomenon". J Appl Physiol, 19:479-482, 1964.

[6] J. A. Hirsch and B. Bishop, "Respiratory sinus arrhythmia in humans: how breathing pattern modulates heart rate". Am J Physiol Heart Circ Physiol 241: H620-H629, 1981.

[7] Brown TE, Beightol LA, Koh J, Eckberg DL, “Important influence of respiration on human R-R interval power spectra is largely ignored". J Appl Physiol 75(5): 2310-2317, 1993.

[8] Guillen Mandujano A., Carrasco Sosa S., "Interactive Effects of Simultaneously Varying Respiratory Frequency and Tidal Volume on Respiratory Sinus Arrhythmia". Computing in Cardiology; 39:165-168, 2012.

[9] Grossman P, Taylor E., “Toward understanding respiratory sinus arrhythmia: relations to cardiac vagal tone, evolution and biobehavioral functions". Biol Psychol; 74:263-85, 2007.

[10] Coello Paola, “Indicador de Actividad Vagal basado en la Arritmia Sinusal Respiratoria", UAM-I, p19, 2007

[11] Task force of the European Society of Cardiology and the North American Society of Pacing and Electrophysiology. "Heart rate variability, standards of measurement, physiological interpretation and clinical use”. Circulation 93:1043-1065, 1996. 
[12] Cabrales Neira Mauricio F., Vanegas Cadavid Diego I., "Manual de métodos diagnósticos en electrofisiología cardiovascular". Sociedad Colombiana de Cardiología y Cirugía Cardiovascular, 15-20, 93-97, 2006.

[13] Echeverry Julian D., Guarnizo Lemus C., Orosco G. Álvaro A., “Análisis de la densidad espectral de potencia en registro MER". Scientia et technica XIII, 035:123-126, 2007.

[14] A. A. Silva, M. A. Irmão, “Comparison of Time-Frequency Representations Applied in simulation and Faults Analysis in Geared Systems". Información tecnológica La Serena, (15)5:17-22, 2004.

[15] Chávez Armando, Tesis Licenciatura: “Evaluación de Microcontroladores”, UAM-I, 2006.

[16] Pomeranz B, Macaulay RJ, Caudill MA, Kutz I, Adam D, Gordon D, Kilborn KM, Barger AC, Shannon DC, Cohen RJ. "Assessment of autonomic function in humans by heart rate spectral analysis". Am J Physiol 248: H151-H153, 1985.

[17] Smythies J., "A note on the concept of the visual field in neurology, psychology, and visual neuroscience". Perception 25 (3): 369-71, 1996.

[18] Jay W. M., “Visual field defects”. American family physician 24 (2): 138-42, 1981.

[19] Guyton Arthur C., Hall John E., “Tratado de fisiología médica”. XI edición, Elsevier Health Sciences, 7:471-482, 2011.

[20] Ortiz Guzman Johan E., Mendoza Romero D., Calderón Carlos A., Urbina A., “Análisis de los componentes espectrales de la variabilidad de la frecuencia cardíaca en hombres jóvenes entrenados: comparación del entrenamiento aeróbico $\mathbf{y}$ anaeróbico". Apunts Medicina d l'Esport, Consell Català de l'Esport, 47:174:41-47, 2011.

[21] Taylo J. A., Myers C. W., Halliwill J. R., Seidel H., Eckberg D. L., "Sympathetic restraint of respiratory sinus arrhythmia: implications for vagal-cardiac tone assessment in humans". Am. J. Physiol heart circ. Physiol, 280, H2804-H2814, 2001.

[22] Larsen P. D., Tzeng Y. C., Sin P. Y., Galletly D. C., "Respiratory sinus arrhythmia in conscous humans during spontaneous respiration". Physiol neurobiol, 174, 111-118, 2010.

[23] Rosado Muñoz A., “Desarrollo de técnicas de detección de fibrilación ventricular basadas en algoritmos de Tiempo-Frecuencia". Universidad de Valencia España, 2:1-3, 2009. 
[24] Saul J. P., Cohen R. J., "Respiratory sinus arrhythmia”. In: Levy M. N., Schwartz P. J., Editores; Vagal Control of the Heart: Experimental Basis and Clinical Implications, Futura Publishing Co. (EU), p. 511-535, 1994.

[25] Malik M. “Heart rate variability”. Current Opinion in Cardiology 13:36-44, 1998.

[26] La Rovere MT, Pinna GD, Maestri R, Mortara A, Capomalla S, Febo O, Ferrari R, Franchini M, Gnemmi M, Opasich C, Riccardi PG, Traversi E, Cobelli F. “Short-Term Heart Rate Variability Strongly Predicts Sudden Cardiac Death in Chronic Heart Failure Patients". Circulation 107:565-570, 2003.

[27] Timbs John. “The year-book of facts in science and art". Lockwood \& Co., 1856.

[28] Kobayashi $\mathrm{H} .$, "Normalization of respiratory sinus arrhythmia by factoring in tidal volume”. Appl. Human Sci., 17, 207-213, 1998.

[29] Eckberg DL. “The human respiratory gate”. J Physiol 548(2):339-352, 2003.

[30] Navarro Gomez M. V., "Enfermería medico quirurgica", 2a edición Elsevier España, ISBN 8445809555, 9788445809556, p. 32-33, 2000.

[31] Palma Jose Luis, "Electrocardiografía de holter: bases prácticas y aplicaciones clínicas”, Capitel editores, ISBN 8474870232, 9788474870237, p. 92, 1993.

[32] Micki Cuppett, Katie M Walsh. "Medicina general aplicada al deporte". EditorElsevier España. ISBN8481749915, 9788481749915. p.183, 2007.

[33] Weissmann, P; Risk, M. R; Giunta, G; Sánchez, Ramiro A; Ramírez, Agustín José. "Control baroreflejo de la frecuencia cardíaca y la variabilidad de la presión arterial: medición a través del análisis computarizado de la señal de presión arterial en sujetos normotensos y pacientes hipertensos". Rev Argent cardiol 65(supl. 3):39-43, 1997.

[34] Novak V, Novak P, De Champlain J, Le Blanc AR, Martin R, Nadeau R. "Influence of respiration on heart rate and blood pressure fluctuations". J Appl Physiol 74:617- 626, 1993.

[35] Proakis J, Manolakis D. “Tratamiento digital de señales, principios, algoritmos y aplicaciones". Prentice Hall México, 3aㅡ ed.

[36] Ghausi-Laker, “Modern filter design”. Prentice Hall México, 1981.

[37] Rodriguez Hernández Miguel P., Cañizares Baña F., Arteaga Gattorno J. L., “El respeto por las personas en la investigación médica un principio básico de bioética". CENTROMED Red telemática de salud, Gaceta médica espirituana, vol. 6 año 3, Cuba 2007. 


\section{Fuentes electrónicas de consulta}

[1] Kits PIC®, Custom Computer Services (CCS) http://www.ccsinfo.com.

[2] Sistemas Arduino, ElectroLabo http://electrolabo.com. 


\section{ANEXO I \\ Ajuste de parámetros fisiológicos}

\section{AI 1Ajuste de parámetros en frecuencia}

La frecuencia de la respiración espontánea en sujetos sanos es de aproximadamente 12 respiraciones por minuto (12 rpm). De esta manera se estableció el supuesto de que todas las respiraciones presentan el mismo ciclo, por lo tanto:

Si hay 12 respiraciones $(r)$ en $60 \mathrm{~s}$.

El factor de conversión (ecuación 1) es:

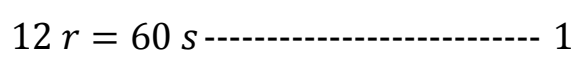

El tiempo de una respiración (ecuación 2) es:

$$
t_{r}=1 r \frac{60 s}{12 r}=5 s
$$

A una respiración le toma aproximadamente cinco segundos en completarse. Esto condujó a establecer otro supuesto, el tiempo de inhalación es igual al tiempo de exhalación, por lo tanto la ecuación 3 refleja este supuesto:

$$
\therefore t_{\text {inspiración }}=t_{\text {espiración }}=t_{r / 2}=2.5 \mathrm{~s} \text {------------- } 3
$$

Estas deducciones son de gran utilidad puesto que establecieron el tiempo base que debe tener el patrón en la columna roja. De tal manera entonces debe tomarle $2.5 \mathrm{~s}$ al indicador subir desde el primero hasta el último en condiciones normales de respiración.

Una vez que se obtuvo el tiempo de inspiración o subida y viceversa, es necesario obtener el tiempo de encendido de cada LED en la columna o vector.

Si enciende en el vector (columna) $70 L E D^{\prime}$ s cada $2.5 s$.

Entonces el factor de conversión (ecuación 4) es:

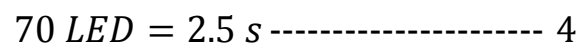


$\therefore$ el tiempo de encendido por LED (ecuación 5) es:

$$
t_{L E D}=1 L E D \frac{t_{r} / 2}{70 \text { LED }}
$$

Sustituyendo los valores en la ecuación 5:

$$
t_{L E D}=1 L E D \frac{2.5 \mathrm{~s}}{70 L E D}=.035 \overline{714285} \mathrm{~s}=35 . \overline{714285} \mathrm{~ms}
$$

Este valor $\left(t_{L E D}\right)$ resultado de la ecuación 5 fue sumamente importante pues determinó el retraso en milisegundos (ms) que debe llevar la programación para cada LED en la función delay() para este caso en particular.

La función usada para obtener el retraso es la función delay() disponible en el entorno Arduino, esta función admite valores en formato largo de tipo doble.

La entrada de usuario se definió en Hertz (Hz) por conveniencia.

La entrada de usuario es en $\mathrm{Hz}$ y el parámetro de la función delay() es en ms, entonces se encontró una relación para el parámetro de entrada de la función delay(), el análisis se describe a continuación.

Cuando se mide la cantidad de respiraciones debido a su larga duración se emplea el minuto como referencia. En tal circunstancia la frecuencia en Hertz es el reciproco de este número de respiraciones con su correspondiente factor de conversión de minutos a segundos. Ver ecuación 6.

$$
\begin{aligned}
& f=N r p m / 60 s \text {--------------------- } 6 \\
& \therefore t=60 \mathrm{~s} / \mathrm{Nrpm}
\end{aligned}
$$

La ecuación de tiempo de retraso por cada LED para la función delay() se obtiene al tomar la mitad del proceso respiratorio, i.e. el tiempo de subida (inhalación) o el tiempo de bajada (exhalación) en el vector.

En la ecuación 3 se establece el supuesto de que el tiempo de inhalación o subida es igual al tiempo de exhalación o bajada. Es decir, el tiempo que toma una respiración dividido entre dos. Ver ecuación 8.

$$
\frac{t}{2}=t_{1 / 2}=30 \mathrm{~s} / \mathrm{Nrpm}-\cdot-\cdot-\cdot---8
$$


Análogamente:

$$
2 f=f_{1 / 2}=N r p m / 30 s \text {------- } 9
$$

De esta manera generalizamos la ecuación para el tiempo de retraso por cada LED $\left(t_{L E D}\right)$. La ecuación 10 generaliza la ecuación 5 referente a un caso particular.

$$
t_{L E D}=\frac{t_{1 / 2}}{V_{L E D}}
$$

Donde $V_{L E D}$ es el tamaño del vector o columna de LED's, siendo una constante con valor de 70.

Al sustituir el valor de $V_{L E D}$ y la ecuación 8 en la ecuación 10, se consigue la ecuación 11 para cualquier valor de entrada en número de respiraciones por minuto (Nrpm):

$$
t_{L E D}=\frac{30 \mathrm{~s}}{\operatorname{Nrpm}(70)}
$$

La entrada de usuario debe ser en Hertz, entonces de la ecuación 9 se resuelve para Nrpm:

$$
N r p m=2 f(30 s)--\cdot-\cdot--------12
$$

Al sustituir la ecuación 12 en la ecuación 11, simplificar y convertir el tiempo en segundos a milisegundos se consigue la ecuación 13 para cualquier valor de entrada en Hertz:

$$
\begin{aligned}
& t_{L E D}=\frac{30 \mathrm{~s}}{2 f(30 \mathrm{~s})(70)} \\
& t_{L E D}=\frac{1}{2 f(70)}=\frac{1}{f} \frac{1}{140}=\frac{1}{f}\left(\frac{1000}{140}\right)=\frac{1}{f} \frac{50}{7} \\
& t_{L E D}=\frac{1}{f} c \text {--------------------- } 13
\end{aligned}
$$

Donde $c=$ constante adimensional de ajuste para la función delay $=\frac{50}{7}$

La ecuación 13 es correcta respecto a las unidades según el siguiente análisis dimensional:

$$
[s]=\frac{1}{\left[\frac{1}{s}\right]}=[s]
$$

La ecuación 13 se usó entonces para realizar la programación de la entrada de usuario en Hz. 
Esta ecuación resolvió el problema planteado en el objetivo referente a tener una amplia programación de frecuencias. Se cubre incluso la banda de frecuencias bajas.

La figura 1 representa la densidad espectral de potencia de un sujeto sano en posición de pie. Esta figura muestra el intervalo de frecuencias típico que se consideró para el diseño del dispositivo.

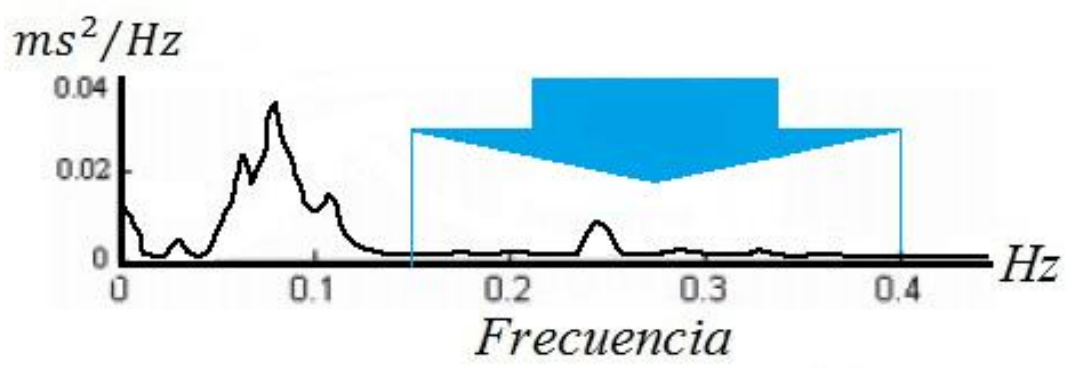

Figura 1. Rango de frecuencias típico.

La ecuación 13 permite programar cualquier frecuencia dentro y fuera de este rango típico. Sin embargo para que estas frecuencias sean consistentes con el proceso fisiológico estas deben ajustarse a un patrón respiratorio determinado.

\section{AI 2 Ajuste del parámetro fisiológico amplitud del volumen respiratorio}

La amplitud del volumen de la respiración humana se determina por medio de un método denominado espirometría. Este método indica los cambios en amplitud del volumen pulmonar por medio de un espirograma.

La teoría define los cambios de la amplitud del volumen respiratorio en cuatro volúmenes y cuatro capacidades.

Los volúmenes pulmonares son:

- Volumen corriente (VC): es el volumen inspirado o espirado en condiciones de respiración normal. Aproximadamente $500 \mathrm{ml}$ en un varón adulto.

- Volumen de reserva espiratoria (VRI): volumen inspirado adicional al VC. Aproximadamente 3,000 ml en un varón adulto.

- Volumen de reserva espiratoria (VRE): volumen espirado forzado después del VC. Aproximadamente 1,100 ml en un varón adulto.

- Volumen residual (VR): volumen que permanece después de una espiración forzada adicional al VC. Aproximadamente 1,200 ml en un varón adulto. 
Las capacidades pulmonares son combinaciones de los volúmenes descritos anteriormente para ayudar a entender mejor los ciclos respiratorios.

Las capacidades pulmonares son:

- Capacidad inspiratoria (CI): cantidad máxima de aire inspirado a partir del nivel espiratorio normal. Aproximadamente 3,500 ml en un varón adulto.

$$
C I=V C+V R I
$$

- Capacidad residual funcional (CRF): $C R F=V R E+V R$ cantidad de aire que permanece al final de una espiración normal. Aproximadamente 2,300 ml en un varón adulto.

- Capacidad vital (CV): $C V=V R I+V C+V R E$ cantidad máxima de aire espirado después de hacer una inspiración máxima de los pulmones. Aproximadamente 4,600 ml en un varón adulto.

- Capacidad pulmonar total (CPT): $C P T=C V+V R$ volumen máximo de expansión de los pulmones.

En mujeres los volúmenes y capacidades son menores en un 20 a $25 \%$.

En el estudio piloto realizado en el Laboratorio se observaron máximos entre 2,000 ml y $3,000 \mathrm{ml}$ para varones y 1,000 a $2,000 \mathrm{ml}$ en mujeres.

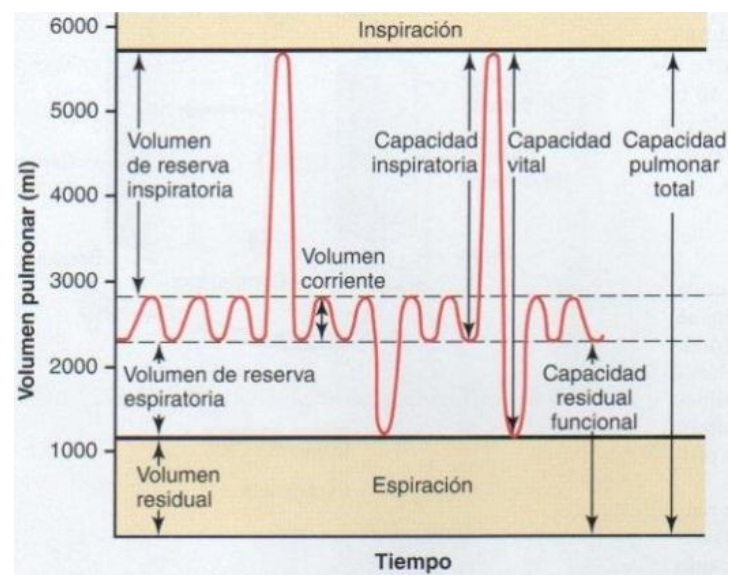

La información extraída del libro de Fisiología Médica (Guyton y Hall) y las observaciones del estudio previo en laboratorio se tomaron para decidir el rango de la amplitud del volumen respiratorio (AVOR).

El rango del GPRV fue de 0 a 3,500 $\mathrm{ml}$ a partir del volumen corriente. Esto quiere decir que sólo se está considerando la capacidad inspiratoria. Ver figura 2 y ecuación 14.

Figura 2. Volúmenes y capacidades pulmonares (Fisiología Médica, Guyton, Hall). 
La resolución (res) del GPRV es entonces de 50 ml por LED como lo indica la ecuación 15.

$$
\begin{aligned}
& \qquad \text { res }=\frac{C I}{V_{L E D}}+15 \\
& C I=\text { capacidad inspiratoria }=3,500 \mathrm{ml} \\
& V_{L E D}=70 \text { LED }
\end{aligned}
$$

Sustituyendo los valores en la ecuación15:

$$
r e s=\frac{3,500 \mathrm{ml}}{70 \mathrm{LED}}=50 \mathrm{ml} / \mathrm{LED}
$$

No existió ambigüedad referente a mantener la frecuencia constante mientras la amplitud es variable. La ecuación 13 resuelve las posibles ambigüedades pues al expandirla ésta queda en términos de la frecuencia y la amplitud:

$$
\begin{aligned}
& t_{L E D}=\frac{1}{f} c= \\
& c=\frac{1}{2\left(V_{L E D}\right)} 1000=\frac{500}{V_{L E D}}
\end{aligned}
$$

Por conveniencia se redefine la constante $V_{L E D}$ que ahora en el caso de amplitud respiratoria variable, $V_{L E D}$ se convierte en una variable, renombrada como: $A_{L E D}$ (Amplitud del volumen respiratorio) y $c_{1}$ es la nueva constante de ajuste para la ecuación 13.

$$
\begin{gathered}
V_{L E D}=A_{L E D}=\text { amplitud del vector de } L E D^{\prime} S \\
\qquad c_{1}=500 \\
\therefore t_{L E D}=\frac{1}{f}\left(\frac{C_{1}}{A_{L E D}}\right)
\end{gathered}
$$

De esta manera la ecuación 13 y sobretodo la 16 son fundamentales en la programación del GPRV.

La ecuación 16 sólo determina el valor máximo deseado en amplitud pero no realiza el decremento en amplitud. Entonces se establece un modelo para realizar el decremento de forma lineal por medio de una recta.

El modelo planteado para la reducción lineal en amplitud se muestra en la figura 3 y en la ecuación 17.

$$
y=m t+b
$$




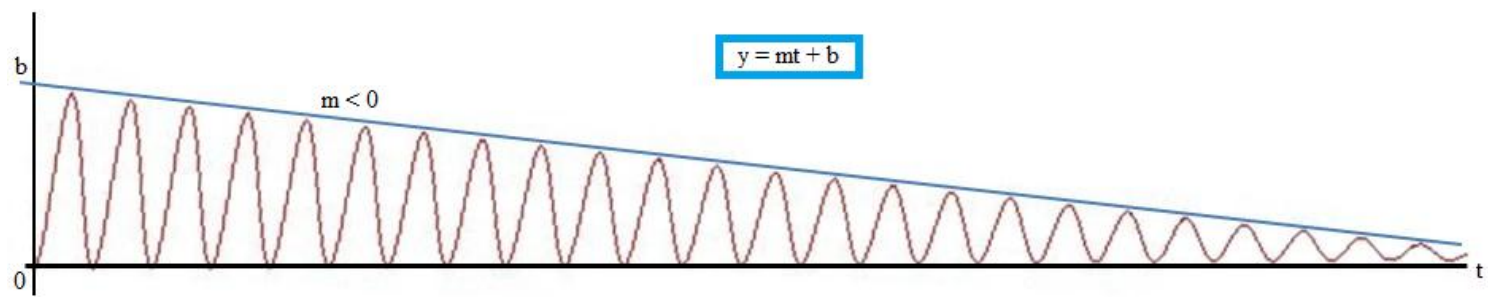

Figura 3. Modelo para decremento de la respiración.

El decremento lineal de la respiración implica que la recta de ajuste tenga una pendiente negativa como se indica en la figura 2.5. La ordenada al origen es entonces el máximo valor del vector de LED's $\left(V_{L E D}=70\right)$. Por lo tanto la ecuación 17 se particulariza para el caso de decremento lineal en:

$$
y=-m t+70
$$

De tal forma que sólo restó calcular el valor de la pendiente:

Para ello se estableció una reducción de $50 \mathrm{ml}$ y otra de $100 \mathrm{ml}$ por cada respiración, esto debido a que los protocolos de esfuerzo respiratorio deben ser no mayores a 6 minutos por razones de fatiga y posibles hiperventilaciones del sujeto.

Entonces con base en la figura 3 y bajo condiciones normales de respiración; 12 respiraciones por minuto; las condiciones iniciales y finales de la variable independiente $t$ y de la variable dependiente amplitud A son las siguientes:

$$
\begin{aligned}
& t_{0}=0 \mathrm{~s} \\
& t_{f}=? \mathrm{~s} \\
& A_{0}=3,500 \mathrm{ml} \\
& A_{f}=0 \mathrm{ml}
\end{aligned}
$$

El tiempo final $t_{f}$ depende de la relación supuesta entre la frecuencia respiratoria por minuto $\mathrm{Nrpm}$, la amplitud $A_{L E D}$ y el tiempo $t$ para condiciones normales de respiración. Observar la tabla 3.3.2-1 que muestra la relación entre $t_{f}, N r p m$ y $A_{L E D}$ para una reducción de $50 \mathrm{ml}$ por respiración. 
Tabla 1. Relación entre las variables $\mathrm{Nrpm}, A_{L E D}$ y $t$ para una reducción en amplitud de $50 \mathrm{ml}$ por respiración.

\begin{tabular}{|l|l|l|}
\hline Nrpm & A [ml] & $\mathbf{t}[\mathbf{s}]$ \\
\hline \hline 1 & 3,500 & 5 \\
\hline 2 & 3,450 & 10 \\
\hline 3 & 3,400 & 15 \\
\hline 4 & 3,350 & 20 \\
\hline 5 & 3,300 & 25 \\
\hline$\ldots$ & $\ldots$ & $\ldots$ \\
\hline N & n & n \\
\hline
\end{tabular}

En la tabla 1 se observa una relación entre las secuencias de las columnas $N r p m, A_{L E D}$ y $t$, dicha relación indica una progresión geométrica. Esta progresión geométrica tiene el comportamiento de la ecuación 18.

$$
a_{n}=a_{1}+(n-1) \mathrm{d}---\cdot------------18
$$

Dónde:

$d$ = una constante resultado de la diferencia de dos términos sucesivos cualesquiera de la secuencia $A_{L E D}$. Y puede ser $d>0, d=0$ ó $d<0$.

$n=$ la posición de cada valor numérico de la secuencia $A_{L E D}$ que para este caso representaría la sucesión de respiraciones Nrpm.

$a_{1}=$ el primer elemento de la sucesión de $A(3,500 \mathrm{ml})$ o de la sucesión de $t$ (5 s).

$a_{n}=$ el elemento por saber en la n-ésima respiración.

Entonces para la 4ta respiración:

$d=\left(A_{4-} A_{3}\right)=3,350 \mathrm{ml}-3,400 \mathrm{ml}=-50 \mathrm{ml}$.

$n=N r p m=4$.

$a_{1}=A_{1}=3,500 \mathrm{ml}$.

$a_{4}=A_{4}$ ?

$$
\begin{aligned}
& a_{4}=3,500+(4-1)(-50) \\
& a_{4}=A_{4}=3,350 \mathrm{ml}
\end{aligned}
$$


Para obtener el valor final $t_{f}$ la ecuación de progresión geométrica debe ser cero lo cual también indica que la amplitud debe estar en su valor mínimo. Esto se resolvió por medio de una ecuación de primer grado que se obtuvo del segundo término de la ecuación 18 . Si este segundo término es igual al primero por el signo negativo de la constante $d$ la ecuación 18 resulta en cero.

$$
\begin{array}{ll} 
& (n-1) \mathrm{d}=-3,500 \mathrm{ml} \\
\text { Conozco d, entonces: } & (n-1)(-50)=-3,500 \mathrm{ml} \\
\text { Resolví para n: } & n=\frac{3,500 \mathrm{ml}}{50 \mathrm{ml}}+1=71 \\
\text { Si } n=71 & a_{71}=3,500 \mathrm{ml}+(71-1)(-50 \mathrm{ml}) \\
a_{71}=3,500 \mathrm{ml}-3,500 \mathrm{ml}=0 \mathrm{ml}=\text { Amplitud cero }
\end{array}
$$

Análogamente para la secuencia de valores de la columna t de la tabla 1, se aplicó el mismo razonamiento y se obtuvó el $t_{f}$ :

$d=10 s-5 s=5 s$.

$n=71$.

$a_{1}=5 s$.

$a_{71}=$ ?

$$
\begin{gathered}
a_{71}=5 s+(71-1)(5 s) \\
a_{71}=5 s+350 s=355 s=t_{f}=5.916 \mathrm{~min}
\end{gathered}
$$

Retomando el análisis del modelo $y=-m t+70$ :

$$
\begin{aligned}
& t_{0}=0 \mathrm{~s} \\
& t_{f}=355 \mathrm{~s} \\
& A_{0}=70 \mathrm{LED} \\
& A_{f}=0 \mathrm{LED}
\end{aligned}
$$

Se calculó la pendiente m: $\quad \quad \quad m=\frac{A_{f}-A_{0}}{t_{f}-t_{0}}=\frac{0-70 L E D}{355-0 \mathrm{~s}}=-0.197183099 \mathrm{LED} / \mathrm{s}$ 
Las unidades de la pendiente son consistentes con el modelo $y=-m t+70$, según el siguiente análisis dimensional:

$$
[L E D]=\frac{[L E D]}{[s]}[s]+[L E D]=[L E D]
$$

Sustituyendo los valores en el modelo y cambiando la variable independiente $\mathrm{t}$ por la ecuación 16 se obtuvó:

$$
\begin{gathered}
y=-.19718309 t_{L E D}+70 \\
y=-.19718309 \frac{1}{f}\left(\frac{C_{1}}{A_{L E D}}\right)+70
\end{gathered}
$$

Donde

$$
\begin{aligned}
& c_{1}=500 \\
& f=\text { frecuencia en } \mathrm{Hz} \text { deseada } \\
& A_{L E D}=\text { Amplitud deseada en } \mathrm{ml}
\end{aligned}
$$

Para el caso de la reducción en amplitud de $100 \mathrm{ml}$ la tabla 3.3.2-2 muestra dichos cambios en su columna A [ml].

Tabla 2. Relación entre las variables $\mathrm{Nrpm}, A_{L E D}$ y $t$ para una reducción en amplitud de $100 \mathrm{ml}$ por respiración.

\begin{tabular}{|l|l|l|}
\hline Nrpm & A [ml] & $\mathbf{t}[\mathbf{s}]$ \\
\hline \hline 1 & 3,500 & 5 \\
\hline 2 & 3,400 & 10 \\
\hline 3 & 3,300 & 15 \\
\hline 4 & 3,200 & 20 \\
\hline 5 & 3,100 & 25 \\
\hline$\ldots$ & $\ldots$ & $\ldots$ \\
\hline $\mathrm{N}$ & $\mathrm{n}$ & $\mathrm{N}$ \\
\hline
\end{tabular}

Usé el mismo modelo:

$$
y=m t+b
$$

Y la ecuación de progresión geométrica: $a_{n}=a_{1}+(n-1) \mathrm{d}$--------- 18

Por lo tanto:

$$
\begin{aligned}
d=3,300 m l-3,400 m l & =-100 m l . \\
& (n-1) \mathrm{d}=-3,500 m l
\end{aligned}
$$




$$
\begin{array}{ll}
\text { Se conoce } d \text {, entonces: } & (n-1)(-100 \mathrm{ml})=-3,500 \mathrm{ml} \\
\text { Se resuelve para } n: & n=\frac{3,500 \mathrm{ml}}{100 \mathrm{ml}}+1=36 \\
\text { Si } n=36 & a_{36}=3,500 \mathrm{ml}+(36-1)(-100 \mathrm{ml}) \\
a_{71}=3,500 \mathrm{ml}-3,500 \mathrm{ml}=0 \mathrm{ml}=\text { Amplitud cero }
\end{array}
$$

Análogamente para la secuencia de valores de la columna t de la tabla 2, se aplicó el mismo razonamiento y se obtuvo el $t_{f}$ :

$d=10 s-5 s=5 s$.

$n=36$.

$a_{1}=5 s$.

$a_{71}=$ ?

$$
\begin{gathered}
a_{71}=5 s+(36-1)(5 s) \\
a_{71}=5 s+175 s=180 s=t_{f}=3 \mathrm{~min}
\end{gathered}
$$

Retomando el análisis del modelo $y=-m t+70$ :

$$
\begin{aligned}
& t_{0}=0 \mathrm{~s} \\
& t_{f}=180 \mathrm{~s} \\
& A_{0}=70 \mathrm{LED} \\
& A_{f}=0 \mathrm{LED}
\end{aligned}
$$

Se calculó la pendiente m: $\quad \quad \quad m=\frac{A_{f}-A_{0}}{t_{f}-t_{0}}=\frac{0-70 \mathrm{LED}}{180-0 \mathrm{~s}}=-0.39 \mathrm{LED} / \mathrm{s}$

Sustituyendo los valores en el modelo se obtuvó:

$$
y=-.39 \frac{1}{f}\left(\frac{C_{1}}{A_{L E D}}\right)+70
$$

Todo el análisis anterior es similar para el caso de incremento en amplitud. La tabla 3 muestra los cambios en la secuencia A para $50 \mathrm{ml}$. 
Tabla 3. Relación entre las variables Nrpm, $A_{L E D}$ y $t$ para un incremento en amplitud de $50 \mathrm{ml}$ por respiración.

\begin{tabular}{|l|l|l|}
\hline Nrpm & A [ml] & $\mathbf{t}[\mathbf{s}]$ \\
\hline \hline 1 & 0 & 5 \\
\hline 2 & 50 & 10 \\
\hline 3 & 100 & 15 \\
\hline 4 & 150 & 20 \\
\hline 5 & 200 & 25 \\
\hline$\ldots$ & $\ldots$ & $\ldots$ \\
\hline N & n & n \\
\hline
\end{tabular}

Comprobando para la $4^{\text {ta }}$ respiración:

$$
a_{n}=a_{1}+(n-1) \mathrm{d}---\cdot-\cdot--\cdot-------18
$$

$d=\left(A_{4-} A_{3}\right)=150 \mathrm{ml}-100 \mathrm{ml}=50 \mathrm{ml}$.

$n=N r p m=4$.

$a_{1}=A_{1}=0 \mathrm{ml}$.

$a_{4}=A_{4}$ ?

$$
\begin{aligned}
& a_{4}=0+(4-1)(50) \\
& a_{4}=A_{4}=150 \mathrm{ml}
\end{aligned}
$$

El valor final $t_{f}$ es cuando la amplitud es máxima:

$$
\begin{aligned}
& 3500=0+(n-1) \mathrm{d} \\
& (n-1) \mathrm{d}=3,500 \mathrm{ml}
\end{aligned}
$$

Conozco d, entonces:

$$
(n-1)(50)=3,500 m l
$$

Resolví para n:

$$
n=\frac{3,500 m l}{50 m l}+1=71
$$

Si $n=71$

$$
a_{71}=0 m l+(71-1)(50 m l)
$$

$a_{71}=0 m l+3,500 m l=3,500 m l=$ Amplitud máxima 
Entonces $t_{f}$ es:

$d=10 s-5 s=5 s$.

$n=71$.

$a_{1}=5 s$.

$a_{71}=$ ?

$$
\begin{gathered}
a_{71}=5 s+(71-1)(5 s) \\
a_{71}=5 s+350 s=355 s=t_{f}=5.916 \mathrm{~min}
\end{gathered}
$$

Por lo tanto el análisis del modelo $y=m t+70$ :

$$
\begin{aligned}
& t_{0}=0 \mathrm{~s} \\
& t_{f}=355 \mathrm{~s} \\
& A_{0}=0 \mathrm{LED} \\
& A_{f}=70 \mathrm{LED}
\end{aligned}
$$

La pendiente m es: $\quad \quad \quad \quad \quad=\frac{A_{f}-A_{0}}{t_{f}-t_{0}}=\frac{70-0 L E D}{355-0 \mathrm{~s}}=0.197183099 \mathrm{LED} / \mathrm{s}$

Sustituyendo los valores en el modelo y cambiando la variable independiente $\mathrm{t}$ por la ecuación 16 se obtuvo:

$$
\begin{gathered}
y=.19718309 t_{L E D}+70 \\
y=.19718309 \frac{1}{f}\left(\frac{C_{1}}{A_{L E D}}\right)+70
\end{gathered}
$$

Donde

$$
\begin{aligned}
& c_{1}=500 \\
& f=\text { frecuencia en } \mathrm{Hz} \text { deseada } \\
& A_{L E D}=\text { Amplitud deseada en } \mathrm{ml}
\end{aligned}
$$

Análogamente el incremento cada $100 \mathrm{ml}$ se muestra en la tabla 4 y en los resultados de las ecuaciones mostradas en seguida de la tabla 4. 
Tabla 4. Relación entre las variables $\mathrm{Nrpm}, A_{L E D}$ y $t$ para un incremento en amplitud de $50 \mathrm{ml}$ por respiración.

\begin{tabular}{|l|l|l|}
\hline Nrpm & A [ml] & $\mathbf{t}[\mathbf{s}]$ \\
\hline \hline 1 & 0 & 5 \\
\hline 2 & 100 & 10 \\
\hline 3 & 200 & 15 \\
\hline 4 & 300 & 20 \\
\hline 5 & 400 & 25 \\
\hline$\ldots$ & $\ldots$ & $\ldots$ \\
\hline N & n & n \\
\hline
\end{tabular}

Comprobando para la $4^{\text {ta }}$ respiración:

$$
a_{n}=a_{1}+(n-1) \mathrm{d}
$$

$d=\left(A_{4-} A_{3}\right)=300 \mathrm{ml}-200 \mathrm{ml}=100 \mathrm{ml}$.

$n=N r p m=4$.

$a_{1}=A_{1}=0 \mathrm{ml}$.

$a_{4}=A_{4}$ ?

$$
\begin{aligned}
& a_{4}=0+(4-1)(100) \\
& a_{4}=A_{4}=300 \mathrm{ml}
\end{aligned}
$$

El valor final $t_{f}$ es cuando la amplitud es máxima:

$$
\begin{aligned}
& 3500=0+(n-1) \mathrm{d} \\
& (n-1) \mathrm{d}=3,500 \mathrm{ml}
\end{aligned}
$$

Se conoce d, entonces: $\quad(n-1)(100)=3,500 \mathrm{ml}$

Se resolvió para n:

$$
n=\frac{3,500 \mathrm{ml}}{100 \mathrm{ml}}+1=71
$$

Si $n=36$

$$
a_{36}=0 m l+(71-1)(50 m l)
$$

$$
a_{36}=0 \mathrm{ml}+3,500 \mathrm{ml}=3,500 \mathrm{ml}=\text { Amplitud máxima }
$$


Entonces $t_{f}$ es:

$d=10 s-5 s=5 s$.

$n=36$.

$a_{1}=5 s$.

$a_{71}=$ ?

$$
\begin{gathered}
a_{71}=5 s+(36-1)(5 s) \\
a_{71}=5 s+175 s=180 s=t_{f}=5.916 \mathrm{~min}
\end{gathered}
$$

Por lo tanto el análisis del modelo $y=m t+70$ :

$$
\begin{aligned}
& t_{0}=0 \mathrm{~s} \\
& t_{f}=180 \mathrm{~s} \\
& A_{0}=70 \mathrm{LED} \\
& A_{f}=0 \mathrm{LED}
\end{aligned}
$$

La pendiente m es: $\quad \quad m=\frac{A_{f}-A_{0}}{t_{f}-t_{0}}=\frac{70-0 \mathrm{LED}}{180-0 \mathrm{~s}}=0.39 \mathrm{LED} / \mathrm{s}$

Sustituyendo los valores en el modelo y cambiando la variable independiente $\mathrm{t}$ por la ecuación 16 se obtuvo:

$$
\begin{gathered}
y=.39 t_{L E D}+70 \\
y=.39 \frac{1}{f}\left(\frac{C_{1}}{A_{L E D}}\right)+70
\end{gathered}
$$

Donde

$$
\begin{aligned}
& c_{1}=500 \\
& f=\text { frecuencia en } \mathrm{Hz} \text { deseada } \\
& A_{L E D}=\text { Amplitud deseada en } \mathrm{ml}
\end{aligned}
$$

Con estos análisis se finaliza el argot matemático. La función 18 puede entonces ser programada para cualquier valor de frecuencia o amplitud del volumen respiratorio. 


\section{AI 3 Simulación de patrones en MatLab}

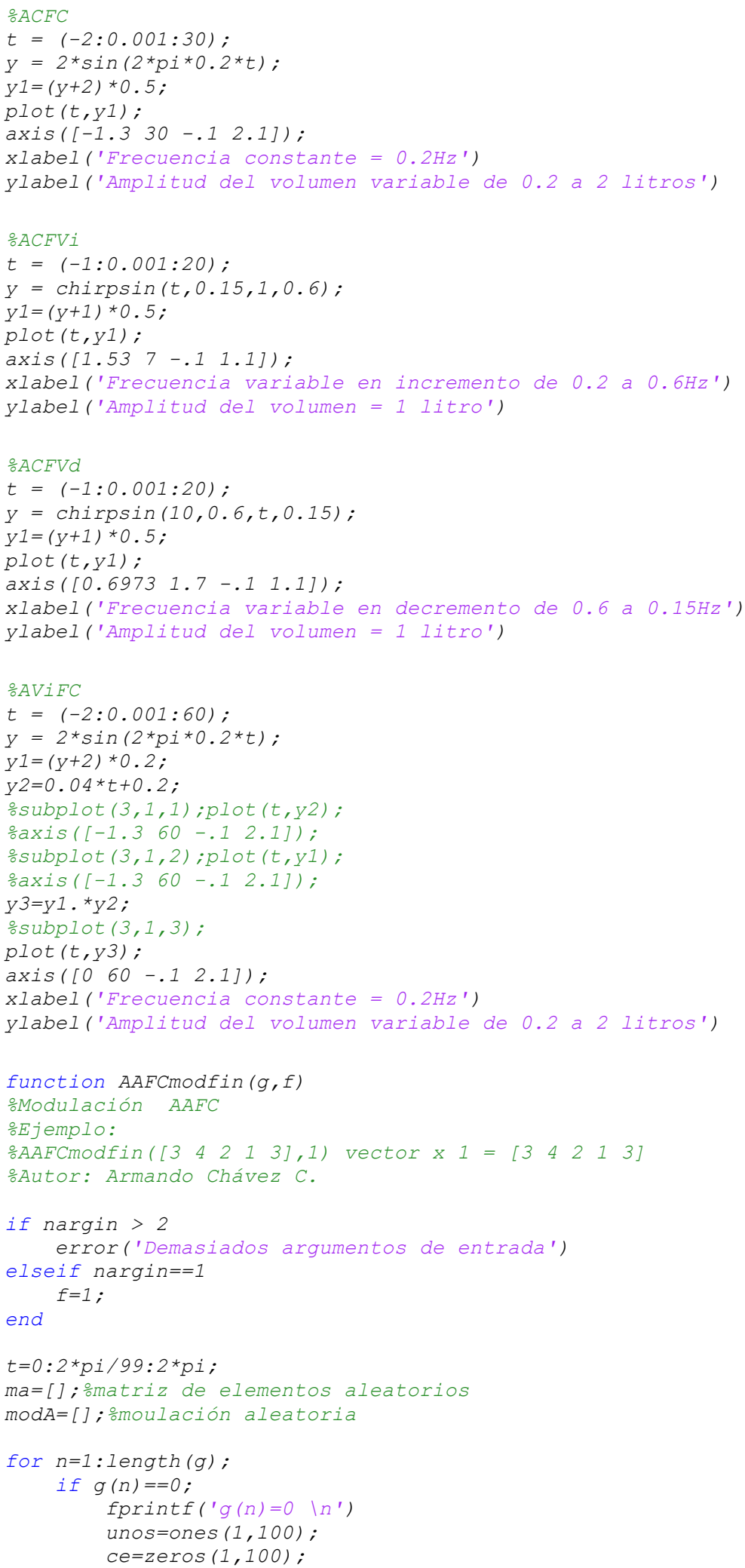




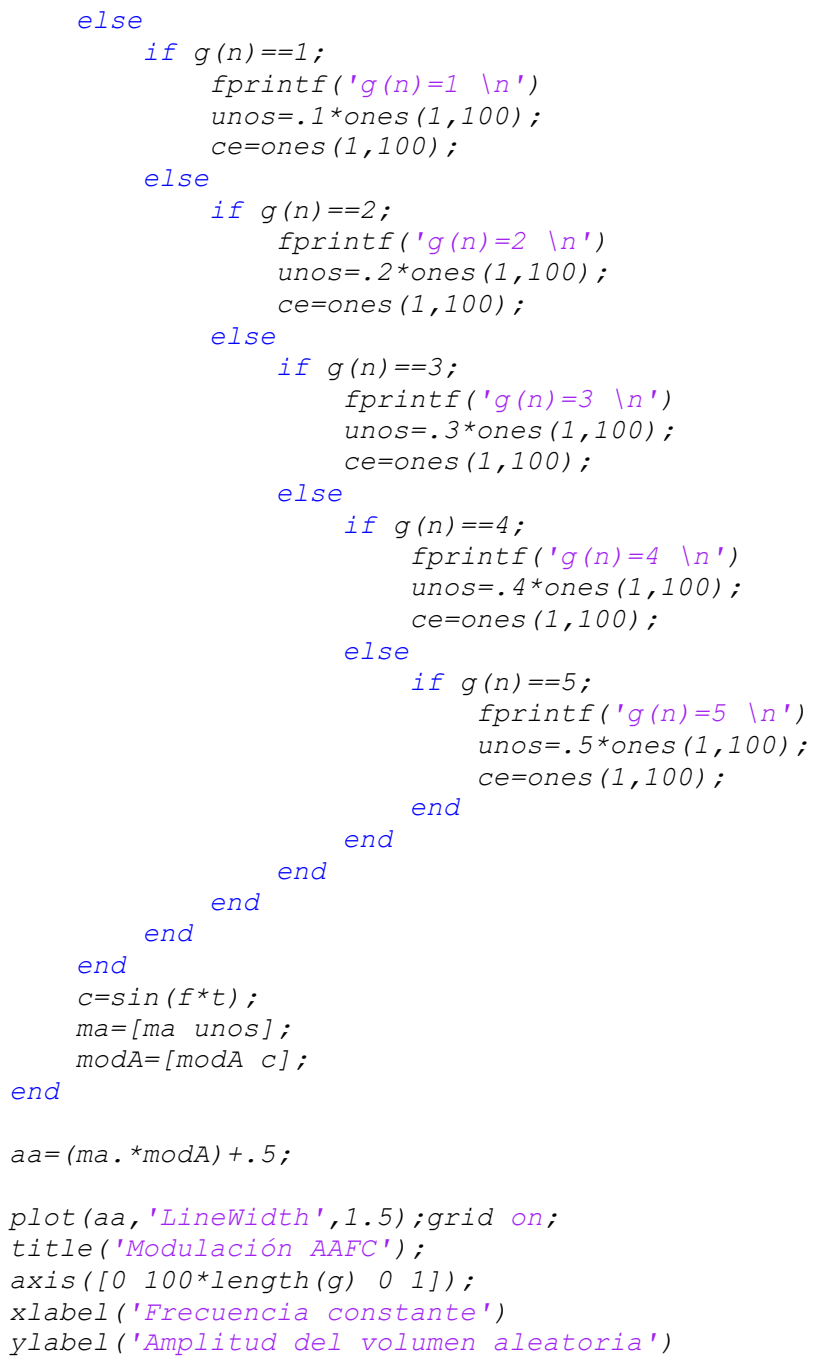

\section{AI 3.1 Otros patrones rspiratorios}

\section{AI 3.1.1 Patrón Amplitud Variable, Frecuencia Variable (AVFV)}

En este patrón existen cuatro casos:

a) Amplitud Variable en decremento, Frecuencia Variable en decremento (AVdFVd)

b) Amplitud Variable en decremento, Frecuencia Variable en incremento (AVdFVi)

c) Amplitud Variable en incremento, Frecuencia Variable en incremento (AViFVi)

d) Amplitud Variable en incremento, Frecuencia Variable en decremento (AViFVd) 
Los parámetros a programar son:

1- Amplitud del volumen inicial y final (Ai, Af) en ml.

2- Frecuencia de respiración inicial y final (Fi, Ff) en $\mathrm{Hz}$.

3- Tiempo de duración del patrón (T) en segundos.

\section{AI 3.1.2 Patrón Amplitud Aleatoria, Frecuencia Constante (AAFC)}

Consiste en generar un patrón aleatorio en amplitud manteniendo una frecuencia constante.

Figura 2.20.

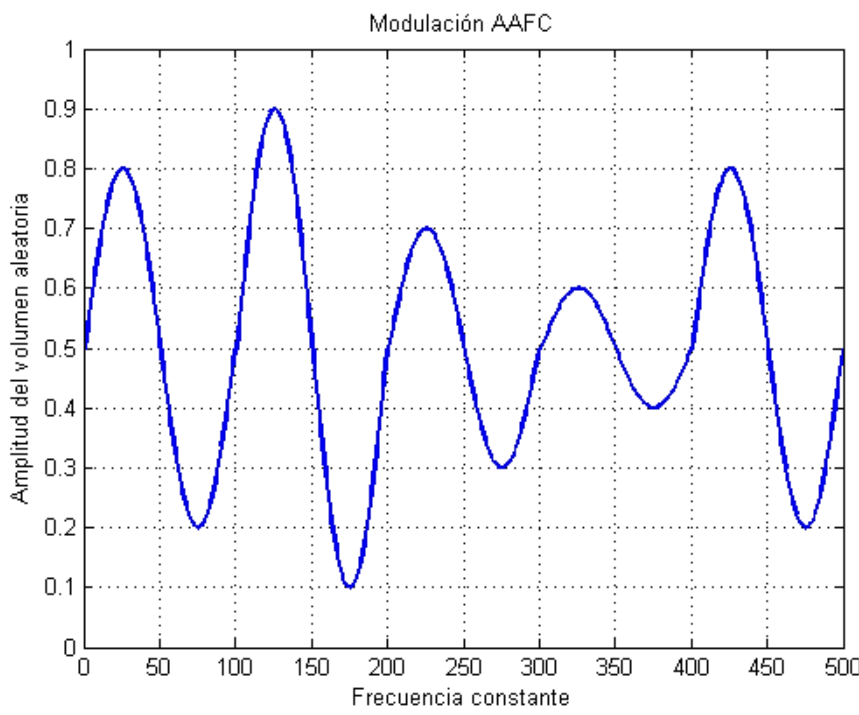

Figura 2.20. Patrón AAFC.

Los parámetros a programar son:

1- Amplitud del volumen (A) en ml.

2- Frecuencia de respiración (F) en $\mathrm{Hz}$.

3- Tiempo de duración del patrón (T) en min.

Todos los patrones se simularon previamente en Matlab ${ }^{\circledR}$, los códigos se pueden observar en el anexol.

\section{AI 4 Patrones respiratorios}

Se muestra el código completo de todos los patrones programado en $\mathrm{C}$ bajo el entorno Arduino. 


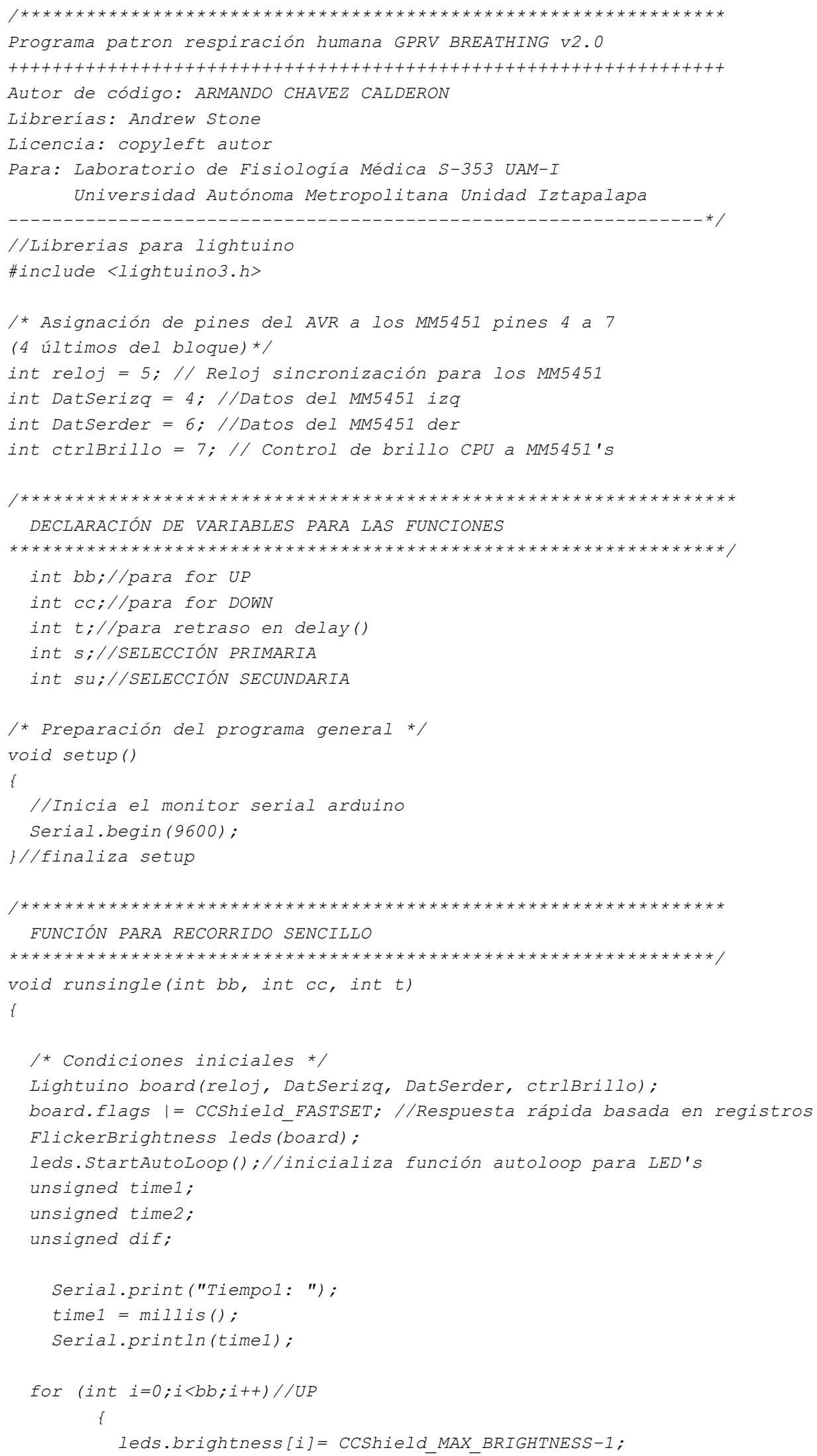




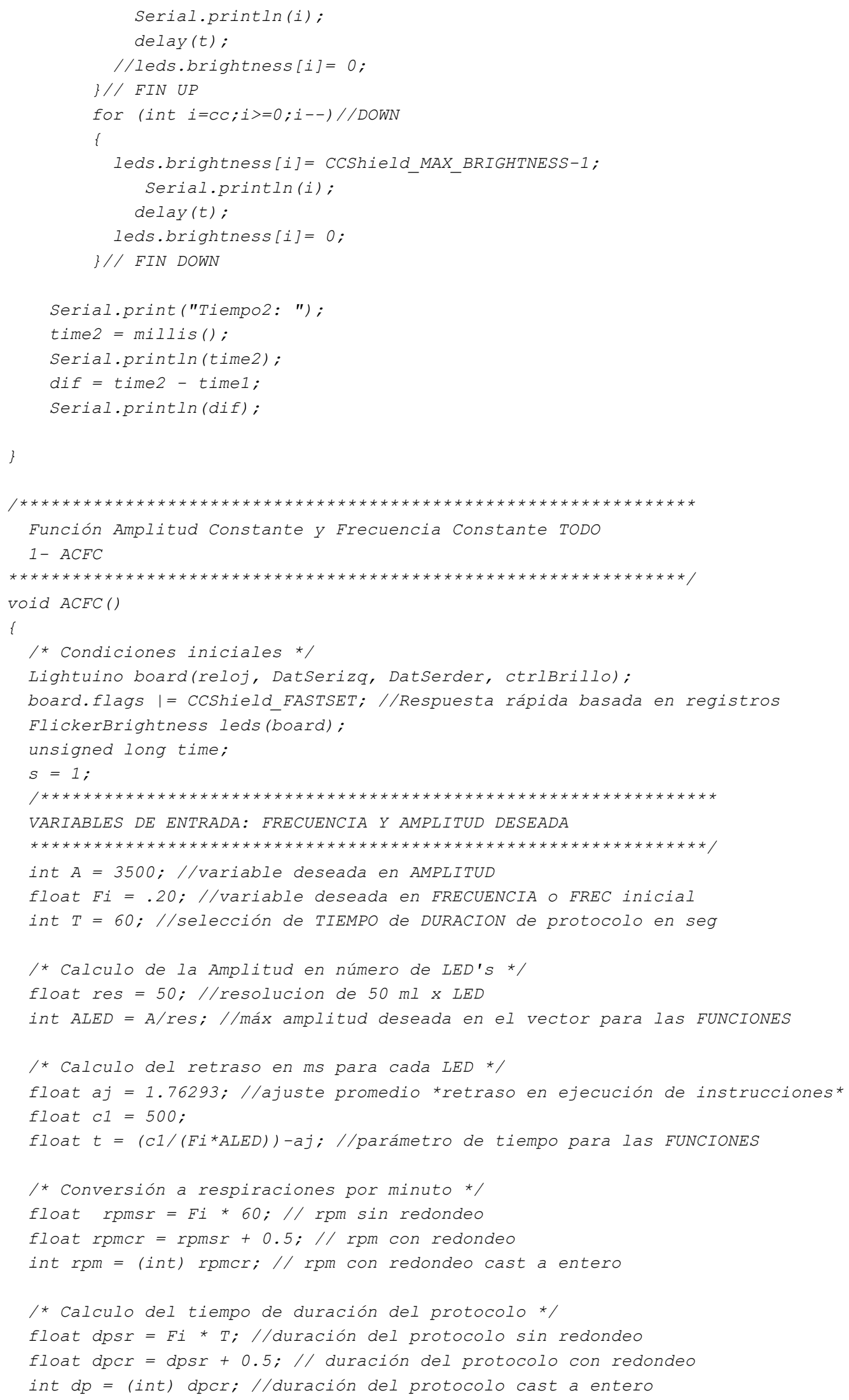




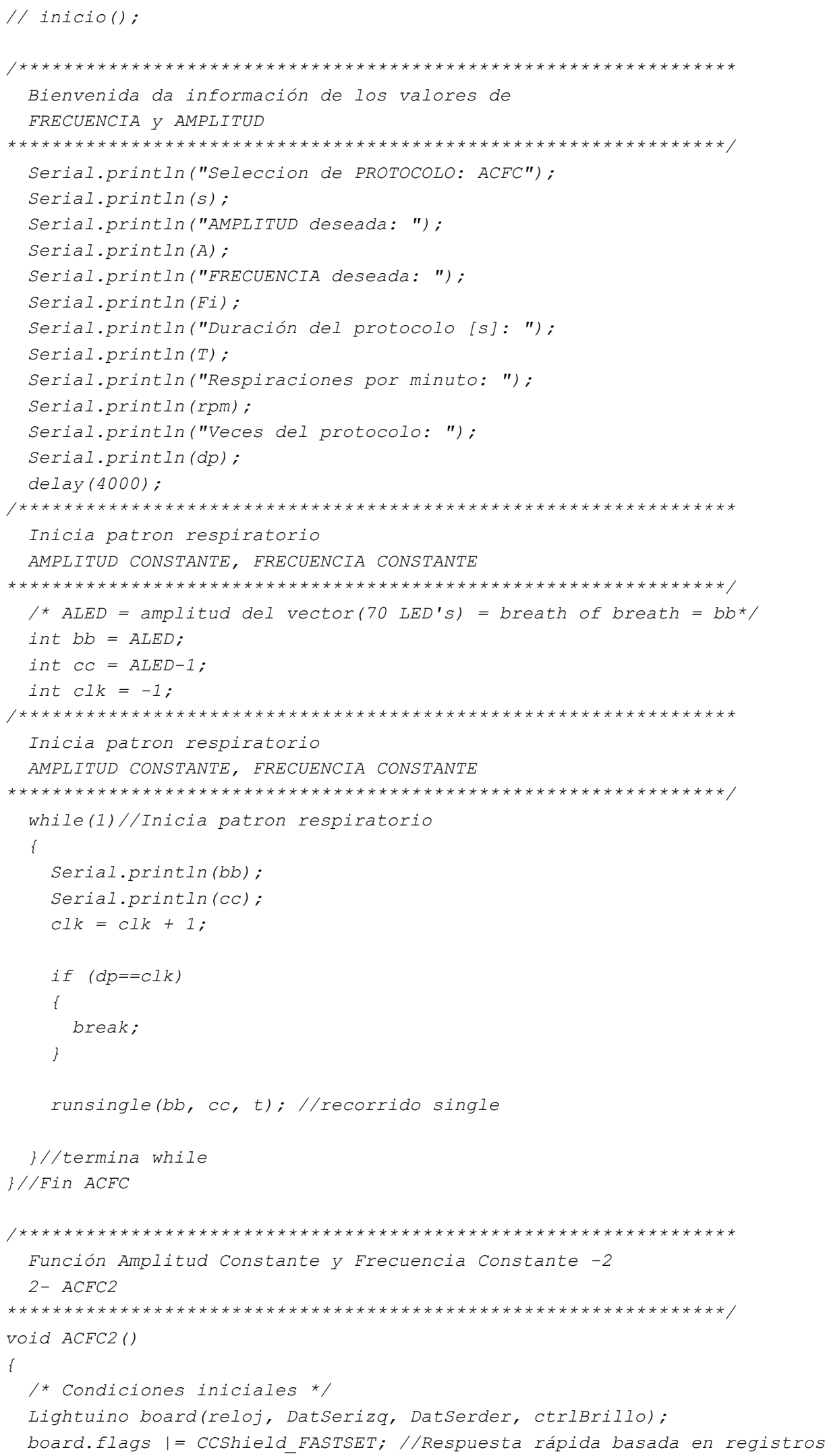




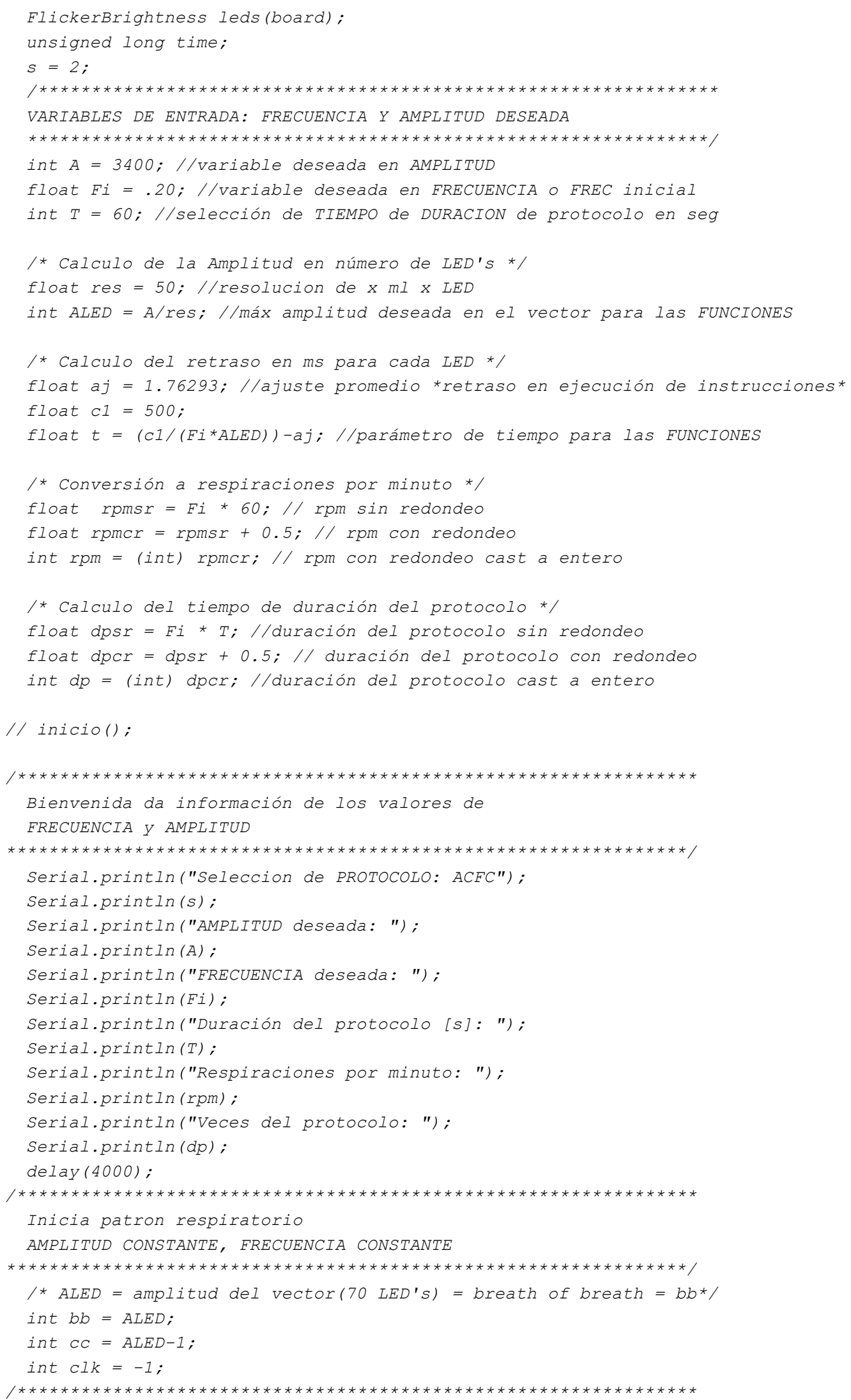




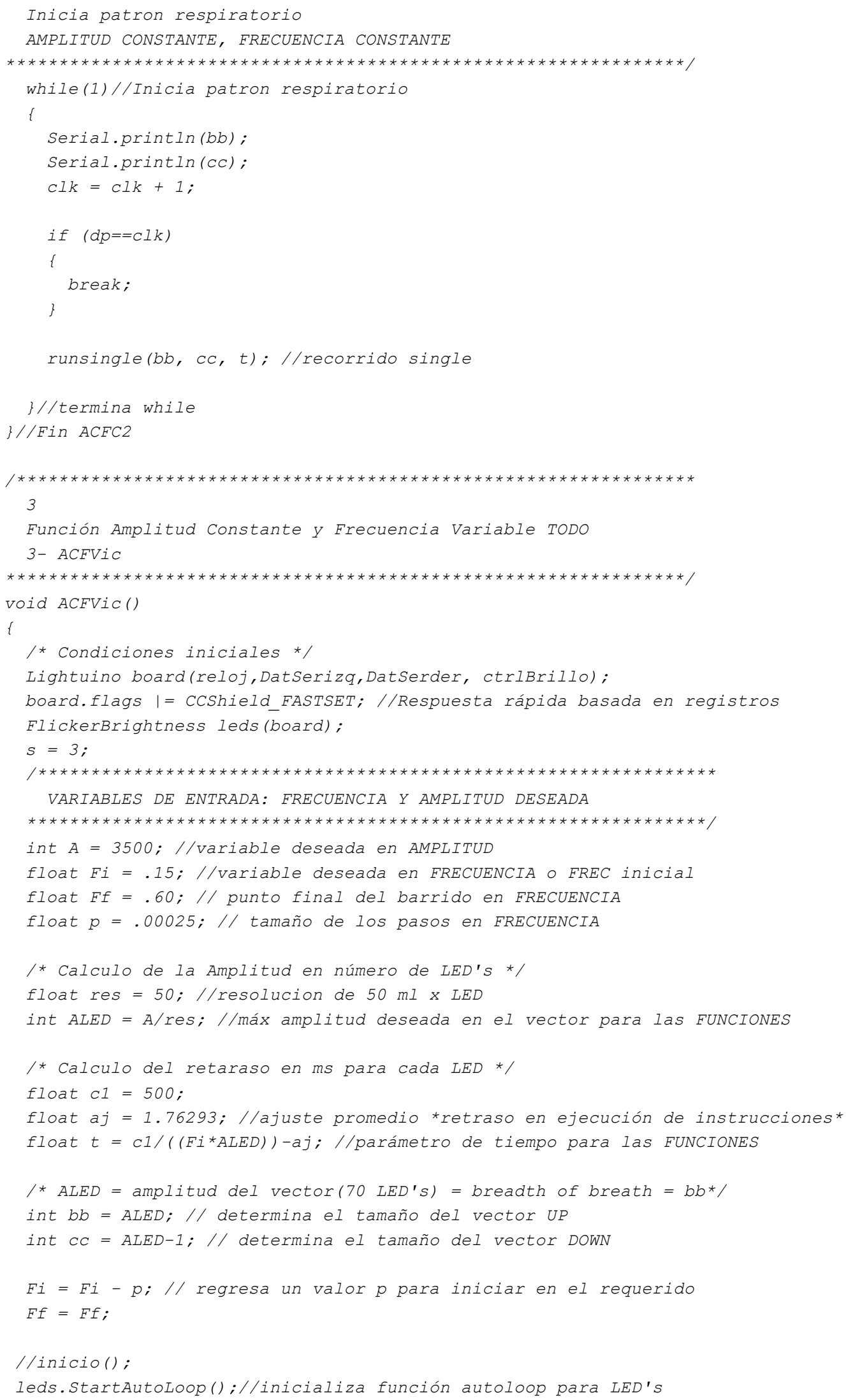




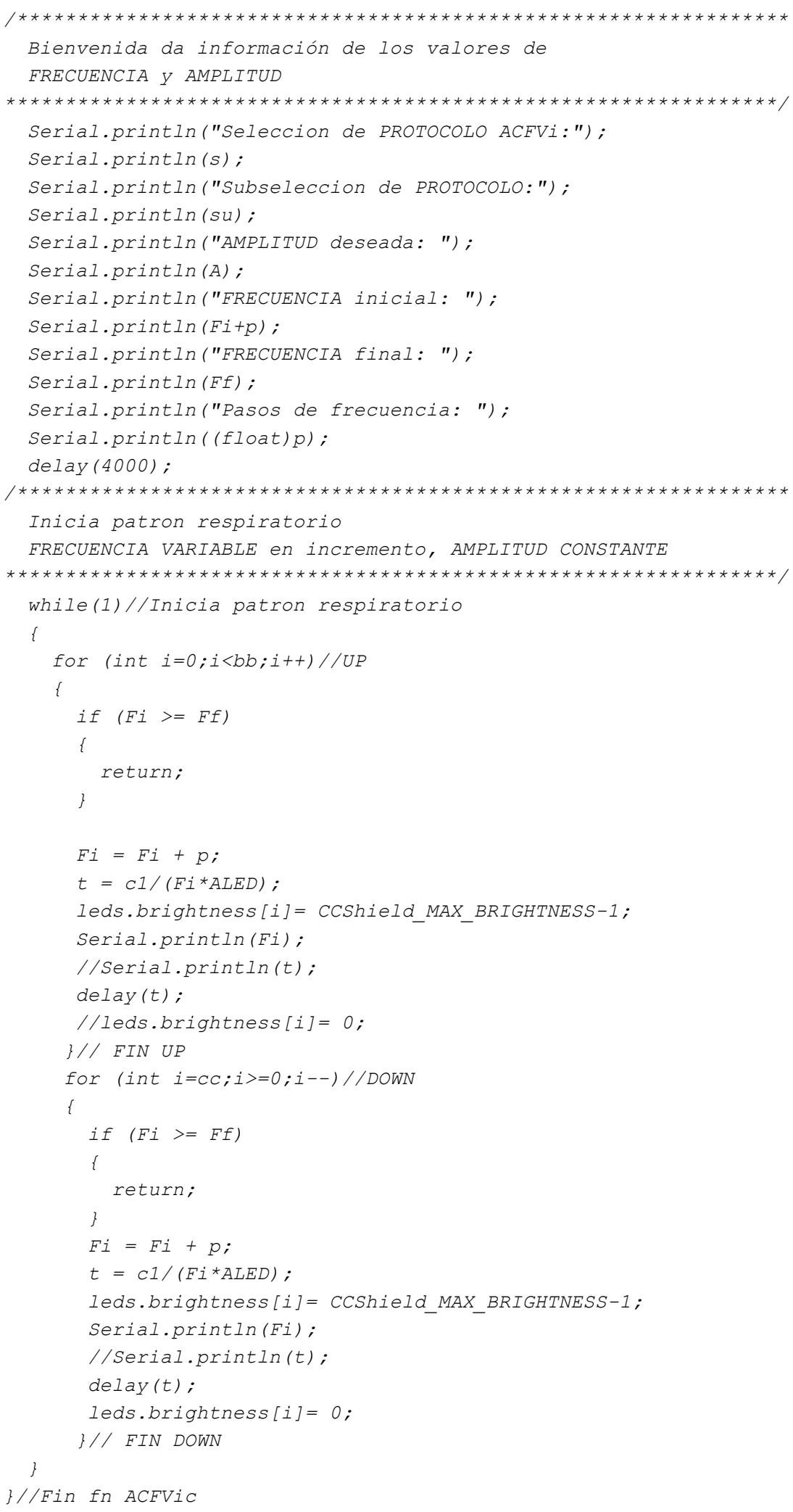




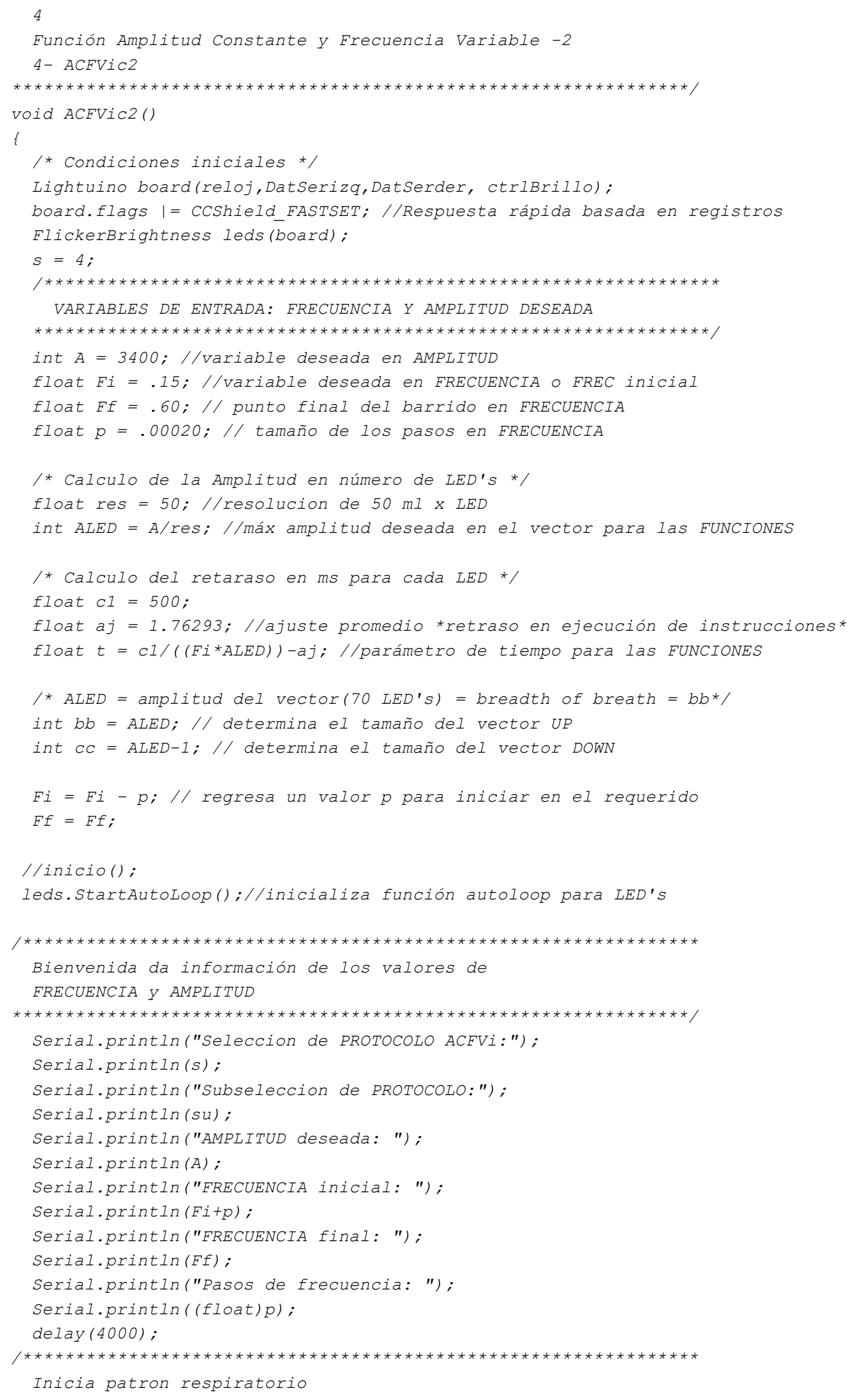




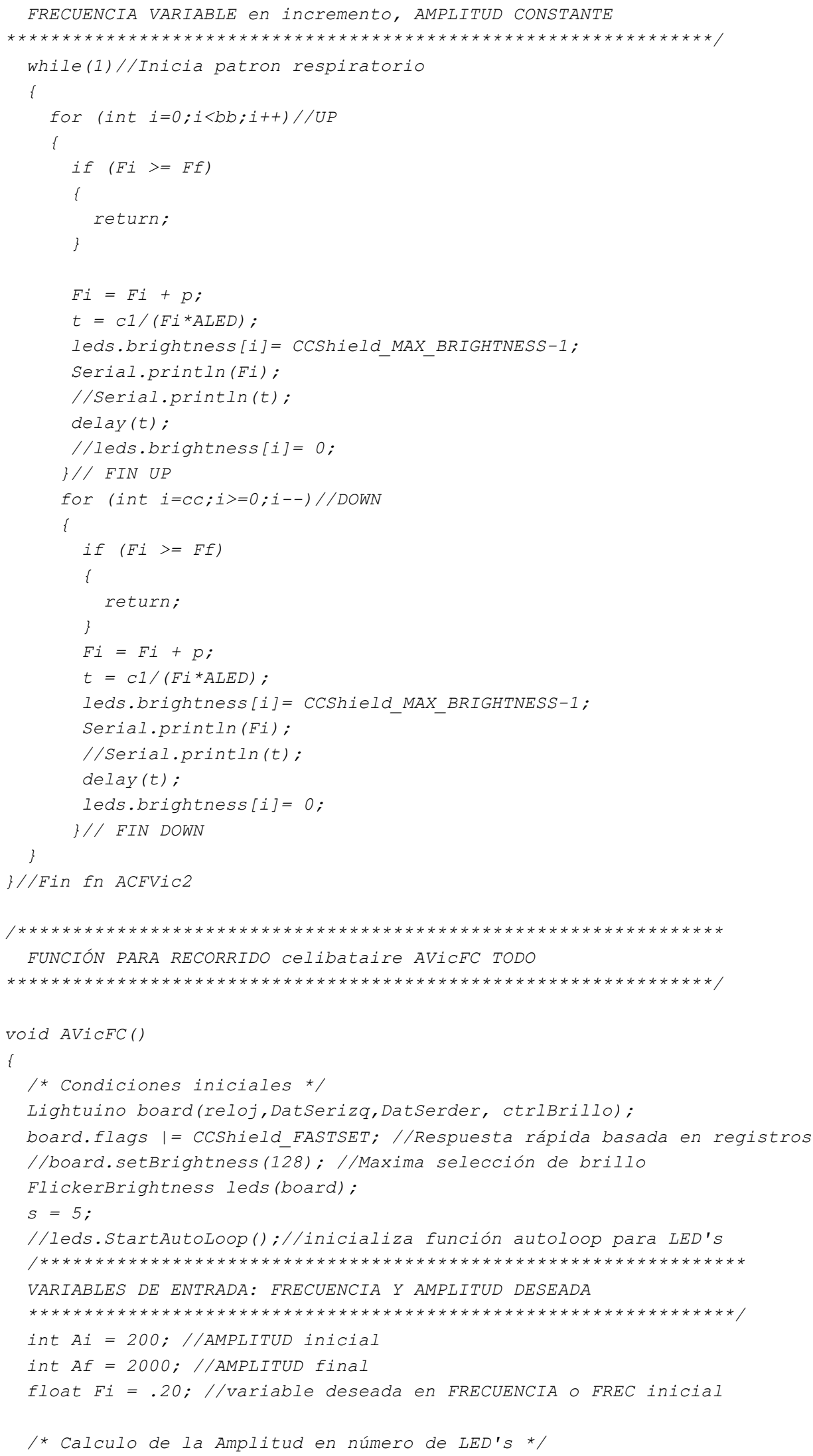




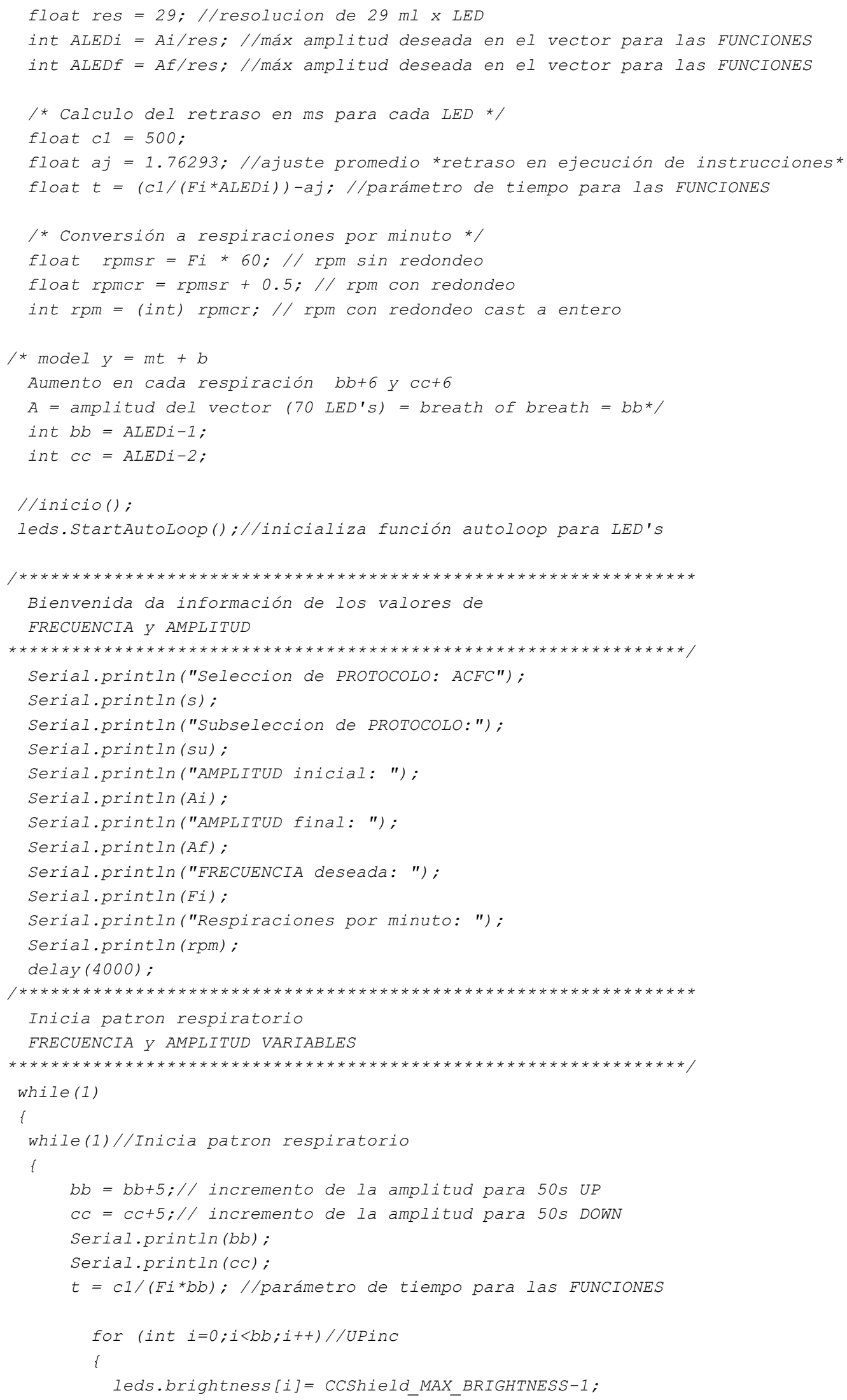




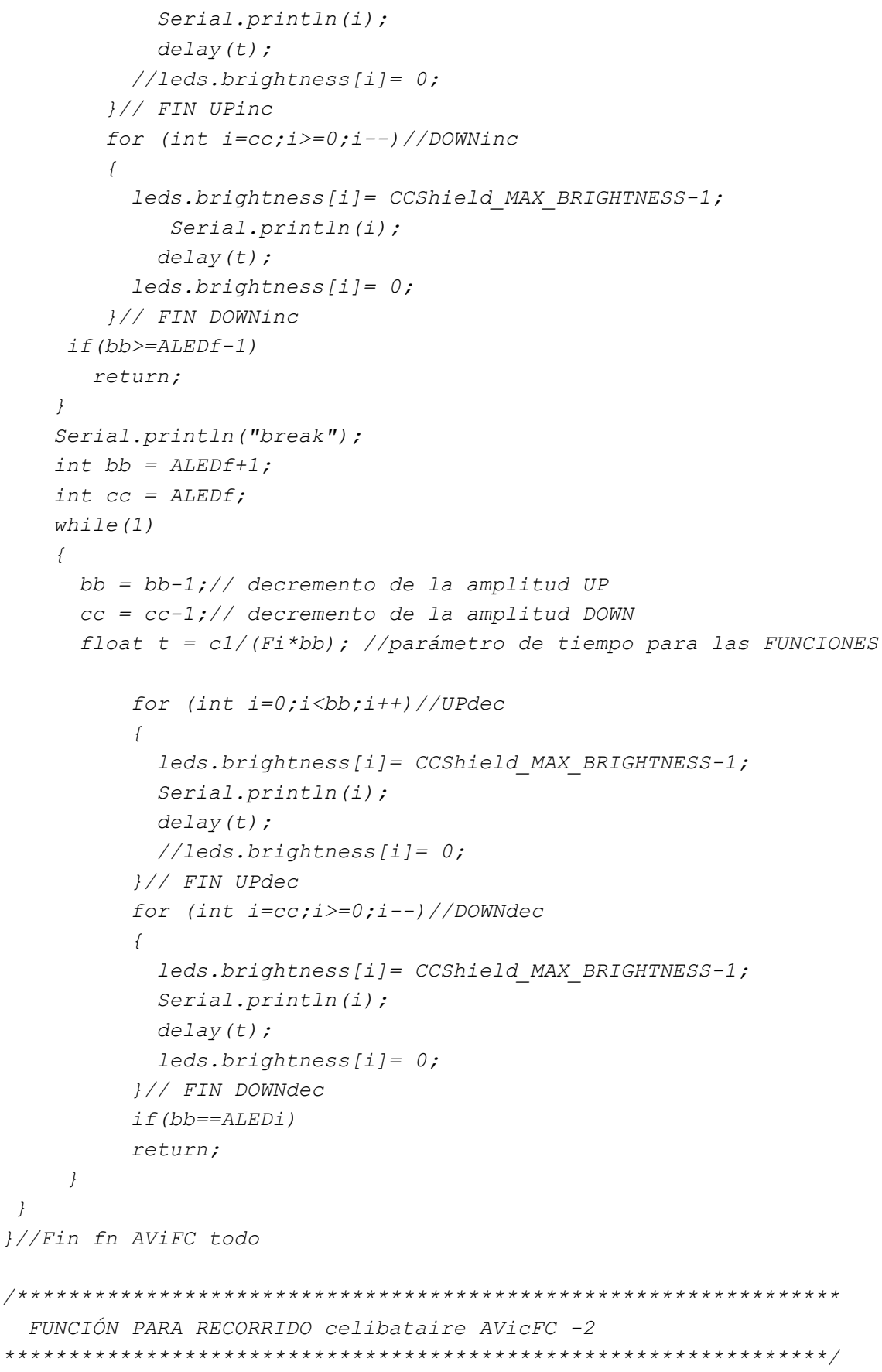




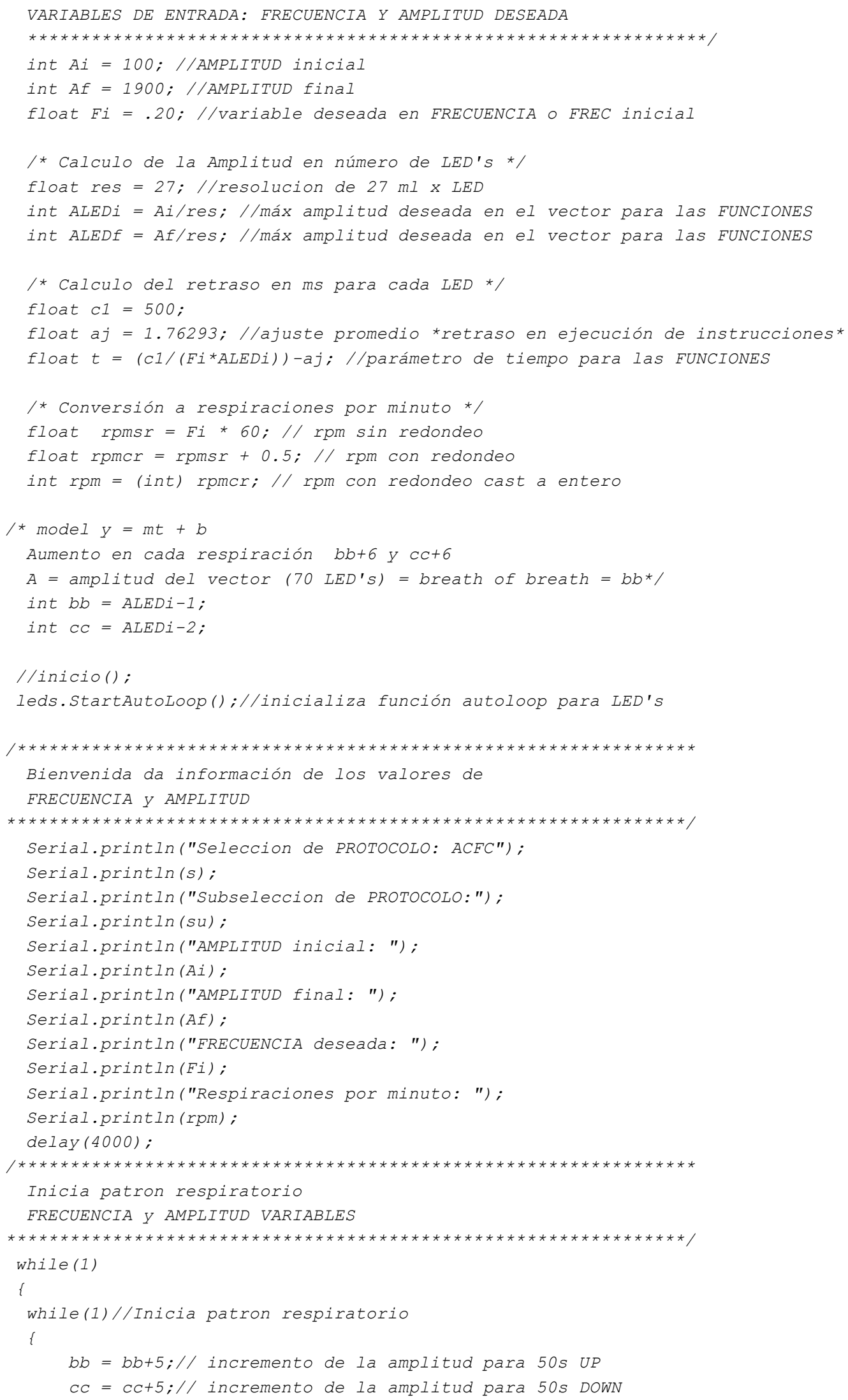




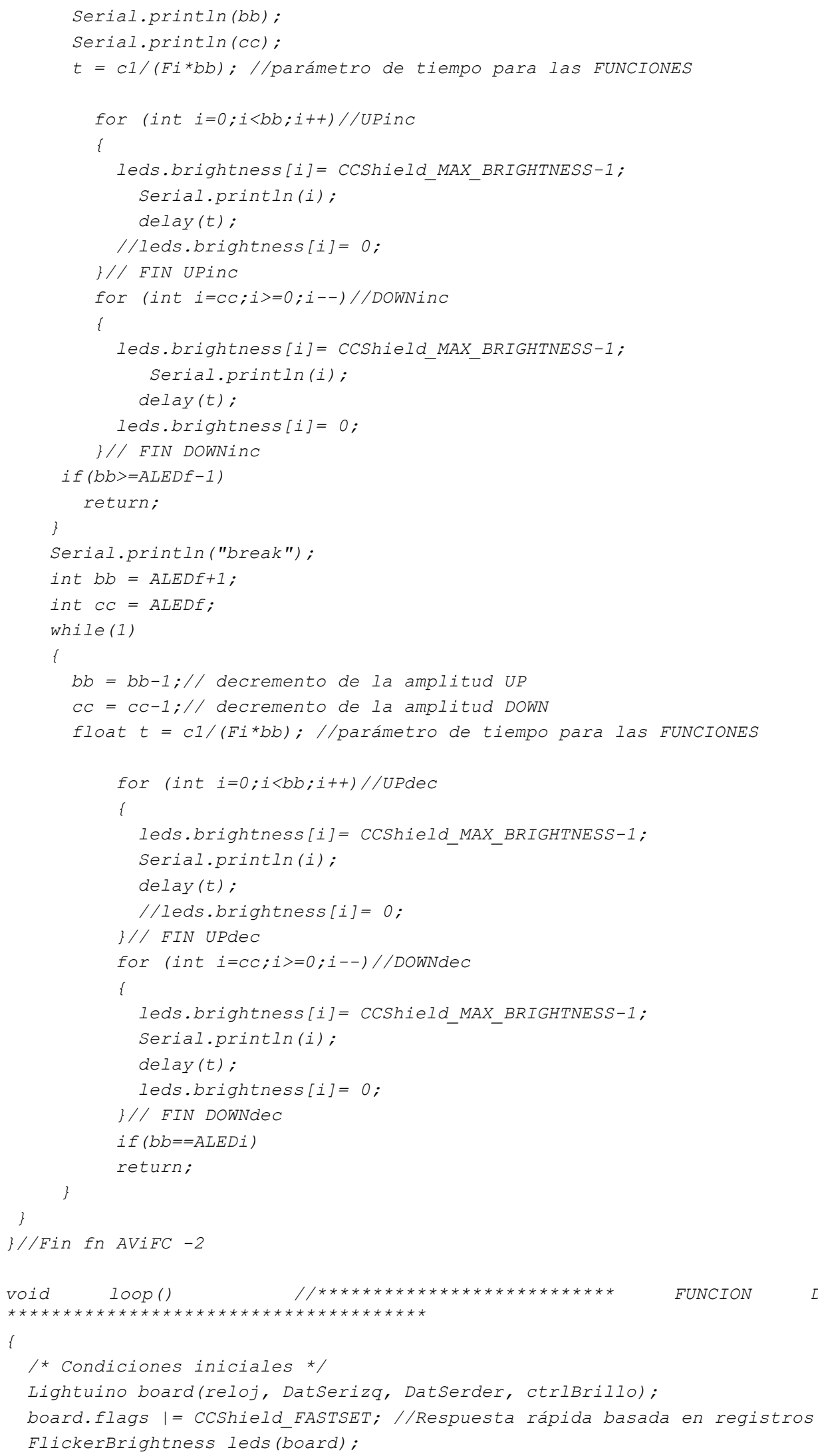




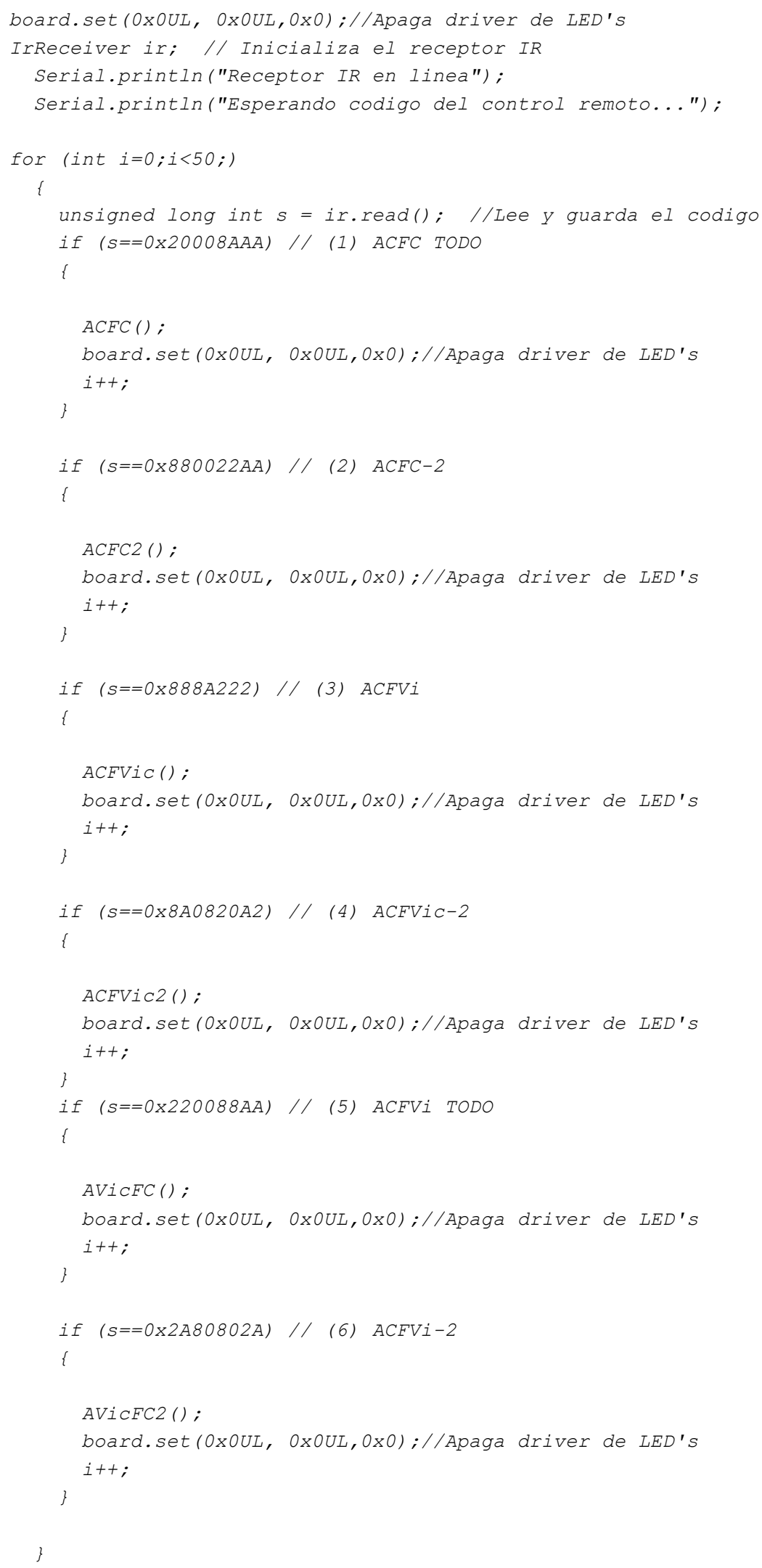




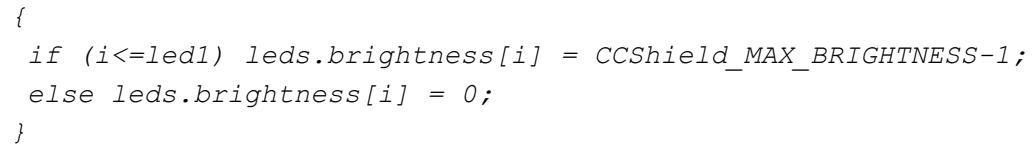




\section{ANEXO II}

\section{AMBIGÜEDAD REFERENTE A MANTENER LA FRECUENCIA CONSTANTE CON RESPECTO A LA AMPLITUD VARIABLE}

En la figura 1 se encuentran dos sinusoides de distintas amplitudes con su correspondiente radio-vector.

La velocidad angular $\omega$ se expresa en términos del periodo $T$ o de la frecuencia $f$, entonces no depende de la amplitud de la onda o el radio vector.

$$
\begin{aligned}
& \omega_{1}=\frac{2 \pi}{T_{1}} \\
& \omega_{2}=\frac{2 \pi}{T_{2}}
\end{aligned}
$$

El radio vector $\mathrm{r}$ debe recorrer la misma distancia que el radio vector $\mathrm{R}$ por lo que es evidente que el tiempo para ambos sea el mismo, como lo indica la ecuación 3.

$$
T_{1}=T_{2}
$$

Esto implica que: $\quad \omega_{1}=\omega_{2}=\omega$------------------ 4

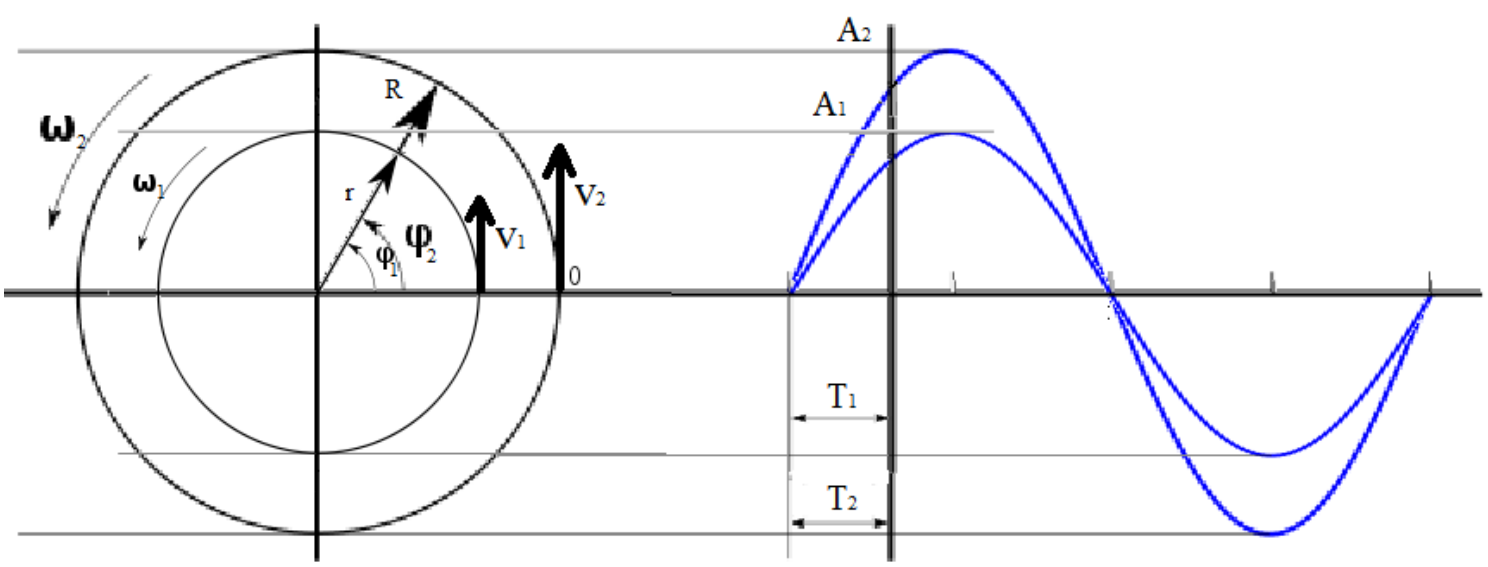

Figura 1. Sinusoides de amplitudes diferentes y su correspondiente radio vector. 
Sin embargo la velocidad tangencial si depende de la amplitud o tamaño del radio vector.

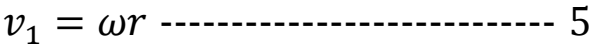

$$
\begin{aligned}
& v_{2}=\omega R
\end{aligned}
$$

De las ecuaciones 5 y 6 se resolvió para $\omega$ y se igualaron ambas:

$$
\frac{v_{1}}{r}=\frac{v_{2}}{R}-\cdot-\cdot-\cdot-\cdot-\cdot-\cdot-\cdot-\cdot------7
$$

A partir de la ecuación 6 se obtuvieron las ecuaciones para ambas velocidades con dependencia de la amplitud:

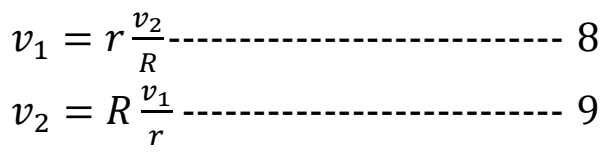

Para comprobar y validar:

Si el radio vector $\mathrm{R}$ fuese el doble que $\mathrm{r}: \quad R=2 r$

Al sustituir la ecuación 9 en las ecuaciones 8 y 9 se obtuvieron los casos particulares:

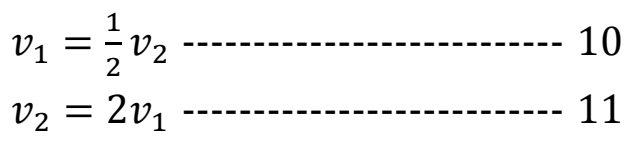

Esto indica claramente que el modelo vector columna del GPRV debe comportarse de la misma forma que las ecuaciones 10 y 11 . Entonces se analizó este caso particular para el modelo vector columna de la siguiente manera (ver figura 2 ):

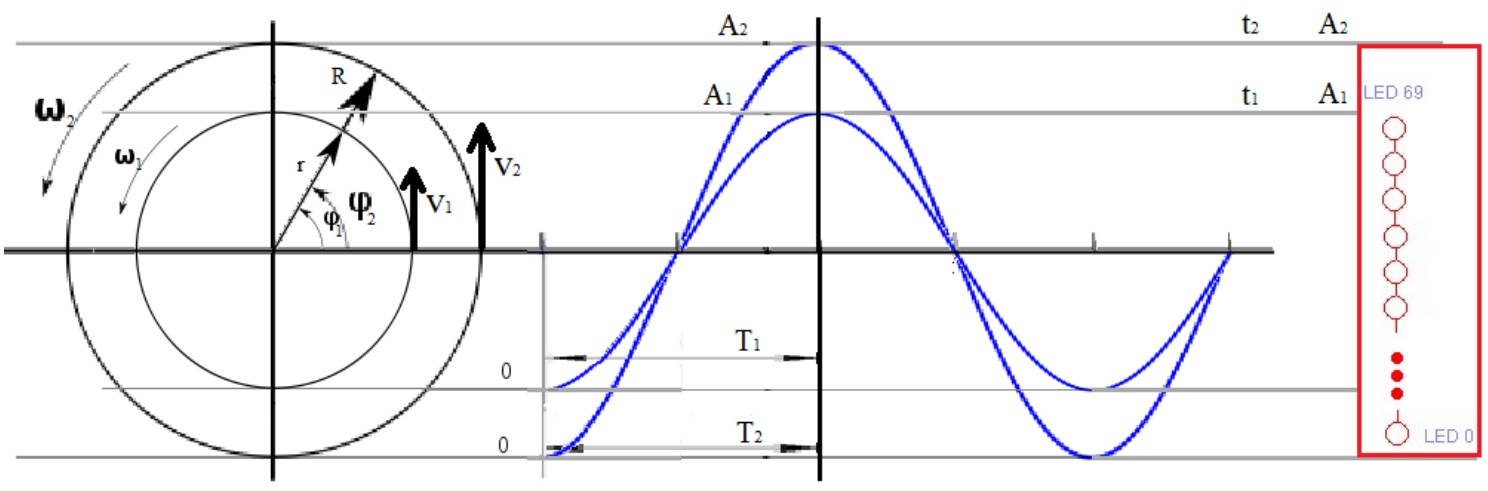

Figura 2. Sinusoide, radio vector y amplitudes del GPRV. 
En el caso del movimiento circular uniforme (MCU) las velocidades tangenciales son distintas y proporcionales respecto al tamaño del radio vector.

En el modelo del GPRV debido al recorrido vertical del indicador de volumen o amplitud respiratoria, a la amplitud $A_{1}$ le toma un tiempo $t_{1}$ mientras que a $A_{2}$ le toma un tiempo $t_{2}$, como en el caso de las velocidades del MCU.

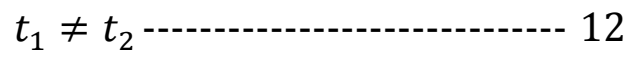

Si las velocidades tangenciales son distintas y proporcionales al tamaño del radio vector, entonces esto traducido al modelo del GPRV implica que para alcanzar una amplitud $A_{1}$ se requiere de una velocidad $v_{1}$ a un tiempo $t_{1}$ y análogamente para $A_{2}$. Por lo tanto si $A_{2}$ es el doble de $A_{1}$ :

$$
\begin{aligned}
& t_{1}=\frac{1}{2} t_{2}-\cdots-12 \\
& t_{2}=2 t_{1}-\cdots---13
\end{aligned}
$$

Si observamos la figura 2, $T_{2}=T_{1}$ esto significa que para que el GPRV alcance la amplitud $A_{1}$ o $A_{2}$ a la misma frecuencia, requiere que el tiempo de recorrido sea el mismo.

Ejemplo:

Sí $T_{2}=2 \mathrm{~s}$ al sustituir en las ecuaciones 12 y 13 :

$$
\begin{aligned}
& t_{1}=\frac{1}{2} 2=1 s \\
& t_{2}=2(1)=2 s
\end{aligned}
$$


